\title{
Hipersuperfícies de Co-homogeneidade 1 do Espaço Hiperbólico
}

\author{
Armando Caputi \\ Tese APRESEnTAda \\ AO \\ InStituto DE MATEMÁtica E EstatísticA \\ DA \\ UNIVERSIDADE DE SÃO PAULO \\ PARA \\ OBTENÇÃO DO GRAU \\ $\mathrm{DE}$ \\ Doutor em MATEMÁtica
}

Área de Concentração: Geometria Diferencial
Orientador: Prof. Dr. Antonio Carlos Asperti

Durante a elaboração deste trabalho, o autor recebeu apoio financeiro do CNPq São Paulo, 21 de dezembro de 2000 


\title{
Hipersuperfícies de Co-homogeneidade 1 do Espaço Hiperbólico
}

\author{
Armando Caputi
}

Este exemplar corresponde à redação final da tese apresentada por Armando Caputi, devidamente corrigida e aprovada pela Comissão Julgadora

São Paulo, dezembro de 2000

\section{Comissão Julgadora}

- Prof. Dr. Antonio Carlos Asperti - IME-USP

- Prof. Dr. José Antonio Verderesi - IME-USP

- Prof. Dr. Francesco Mercuri - IMECC-UNICAMP

- Prof. Dr. Ruy Tojeiro de Figueiredo Júnior - UFSCar

- Prof. Dr. José Adonai Pereira Seixas - UFAL 
À memória dos professores

Franco Tricerri e Seiji Hariki 


\section{Agradecimentos}

Ao meu orientador Antonio Carlos 'Kiko' Asperti, ao professor, ao companheiro, à pessoa, por seu inestimável zelo na orientação, por sua capacidade (e paciência) de conviver com nossas diferenças, pelo apoio e confiança que nunca faltaram nesses anos todos e, não poderia deixar de notar, por seu finíssimo gosto futebolístico.

À alegre e sempre ativa companheira Rosa, por tantos e incontáveis bons momentos, pelo constante apoio que sempre me deu e, principalmente, por sua amizade.

Al grandissimo Franco, per avermi inconsapevolmente riportato a casa e per avermi sempre guidato e ispirato su questa lunga strada.

A todo o grupo de geometria, de São Paulo, Campinas e São Carlos, por todos esses anos de ótima matemática e de maravilhosas bobagens ditas nos inesquecíveis almoços quinzenais.

A todos aqueles que, longe dos holofotes, tocam esse instituto pra frente: seus funcionários. Em particular, ao pessoal da biblioteca, da secretaria do MAT e da CPG, Francisca, Feijão e o grande malabarista da burocracia universitária, Pinho (o que seria da pós sem você?). Um agradecimento com muito carinho a três pessoas muito especiais para todos daqui: dona Maria, Jô e Dalvina (de nada adiantaria tanto pensar, se não houvesse o vosso café para tomar).

Aos companheiros e colegas de todos esses anos, por todas nossas histórias, conversas, idéias, piadas, discussões e até pequenas batalhas; por agüentarem esse chato de carteirinha, retribuindo essa chatice com muito afeto; por to- 
dos os bons e maus momentos compartilhados. Àqueles com os quais dividi intimidades geométricas, em especial à Dulce, Pablo, Zé, Bárbara e Regina, além dos "campineiros" Leonardo (alé viola), Martha, Zé Carlos e Ryuichi (futuro colega) e ao "são carlense" Guillermo. Aos especiais e únicos Raul e Samuel e aos gauchíssimos Alice e Leandro. Aos vizinhos latinos já um pouco brasileiros e ao muitíssimo brasileiro Major. Aos muitos, enfim, que tornaram esses anos tão agradáveis. Um abraço especial e cúmplice ao Daniel, Liane, Cecilia, Irene (e novamente ao Zé) e Paulo, por ainda se indignarem diante da mesquinhez humana.

Um agradecimento sentido àqueles que ainda resistem contra o vergonhoso desmantelamento da universidade pública.

À minha filha Mônica, pelo amor que por ela sinto.

À grande mulher e companheira Cláudia, por existir, por estar, por ser, por toda a força e presença; pelas coisas inomináveis, inexplicáveis, que somente nossos olhos compreendem; por tudo aquilo que somente o silêncio pode exprimir com sinceridade; pelo amor, pelo ardor, pela intensidade, tamanha intensidade que resgata meu choro de menino; pela minha vida.

A todos vocês, mais do que com áridas páginas de matemática, prefiro agradecer com uma poesia de Elisa Lucinda.

o poema do semelhante

o deus da parecença

o que nos costura em igualdade

o que nos papel-carboniza

em sentimento

que nos pluraliza

que nos banaliza

por baixo e por dentro,

foi esse deus que deu

destino aos meus versos, 
foi ele quem arrancou deles

a roupa de indivíduo

e deu-lhes outra de indivíduo

ainda maior, embora mais justa.

me assusta e acalma

ser portadora de várias almas

de um só som comum eco

ser reverberante

espelho, semelhante

ser a boca

ser a dona da palavra sem dono

de tanto dono que ela tem.

esse deus sabe que a palavra "alguém" é apenas

o singular da palavra "multidão".

eh mundão:

todo mundo beija

todo mundo almeja

todo mundo deseja

todo mundo chora

alguns por dentro

alguns por fora

alguém sempre chega

alguém sempre demora.

o deus que cuida do

não-desperdício dos poetas

deu-me essa festa

de similitude

bateu-me no peito do meu amigo

encostou-me a ele

em atitude de verso beijo e umbigos, extirpou de mim o exclusivo:

a solidão da bravura

a solidão do medo

a solidão da usura

a solidão da coragem

a solidão da bobagem

a solidão da virtude 

a solidão da viagem
a solidão do erro
a solidão do sexo
a solidão do zelo
a solidão do nexo.
o deus soprador de carmas
deu de eu ser parecida
aparecida
santa
puta
criança
deu de me fazer
diferente
pra que eu provasse
da alegria
de ser igual a toda gente
esse deus deu coletivo
ao meu particular
sem eu nem reclamar
foi ele, o deus da par-essência
o deus da essência par.
não fosse a inteligência
da semelhança
seria só meu o meu amor seria só minha a minha dor bobinha e sem bonança
seria sozinha minha esperança 


\section{Resumo}

Em [PoSp], foi provado que uma hipersuperfície compacta de dimensão $n \geq 4$ do espaço euclidiano, sobre a qual age um grupo compacto de isometrias com co-homogeneidade 1 e órbitas principais umbílicas, é uma hipersuperfície de revolução. Em [Se], a hipótese de compacidade da variedade foi enfraquecida: o resultado anterior foi estendido a hipersuperfícies completas com um certo controle sobre a planaridade (introduziu-se o conceito de "não-planaridade no infinito"). No nosso trabalho, estendemos o resultado de [Se] a hipersuperfícies do espaço hiperbólico, obtendo um teorema similar com alguns exemplos a mais (cf. Teorema 3.9). 


\section{Abstract}

Let $M^{n}$ be a cohomogeneity one Riemannian manifold and let $f: M^{n} \rightarrow$ $\mathbb{R}^{n+1}$ be an isometric immersion. In [PoSp], for $M$ compact and $n \geq 4$, it was proved that if the principal orbits are umbilical in $M$, then $f$ is a hypersurface of revolution. In [Se] this result was extended for complete hypersurfaces with dimension $n \geq 3$, assuming further a reasonable hypothesis on the flat portion of the manifold $M$ (namely, the hypothesis of "non-flatness at infinity"). Our purpose is to extend the above theorems for hypersurfaces of the hyperbolic space. We prove a similar result of [Se], obtaining also a class of non-rotational examples (see Theorem 3.9). 


\section{Sumário}

$\begin{array}{ll}\text { Introdução } & 3\end{array}$

1 Conceitos Básicos $\quad 6$

1.1 Imersões Isométricas . . . . . . . . . . . . . . . . . . 7

1.1.1 Imersões umbílicas . . . . . . . . . . . . . 9

1.1.2 Teoremas de rigidez . . . . . . . . . . . . . . . 10

1.1 .3 Homotetias . . . . . . . . . . . . . . . 11

1.2 Produtos Torcidos . . . . . . . . . . . . . . . 12

1.2.1 Tensor de curvatura de um produto torcido . . . . . 14

1.3 Grupos de Transformações . . . . . . . . . . . . . . . . . . 16

1.3.1 Ações de co-homogeneidade 1 . . . . . . . . . . 21

2 Geometria Hiperbólica 24

2.1 Espaço de Lorentz . . . . . . . . . . . . . . . . . . . 25

2.2 Espaço Hiperbólico . . . . . . . . . . . . . . . . . . . . . . 30

2.2 .1 Subvariedades Umbílicas . . . . . . . . . . . . . 31

2.3 Funções Distância . . . . . . . . . . . . . . . . . . . 35

2.3.1 Umbílicas como conjuntos de nível . . . . . . . . . 39

2.3.2 Imersões contidas em hipersuperfícies umbílicas . . . 41

2.4 Hipersuperfícies de Revolução . . . . . . . . . . . . . . . . . . 43

$2.5 \mathbb{H}^{n}$ como Produto Torcido . . . . . . . . . . . . . . . 47

3 Hipersuperfícies de Co-homogeneidade $1 \quad 49$

3.1 Hipersuperfícies Homogêneas . . . . . . . . . . . . . . . . 51

3.2 Discussão Preliminar . . . . . . . . . . . . . . . . . . . 52

3.3 Exemplos Não Rotacionais . . . . . . . . . . . . . . . . 54

3.4 Teorema Principal . . . . . . . . . . . . . . . 56

3.5 Demonstração . . . . . . . . . . . . . . . . . . 61 


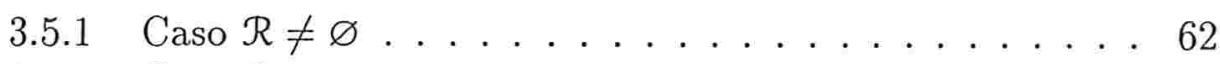

3.5.2 Caso $\mathcal{R}=\varnothing \ldots \ldots \ldots \ldots 78$

3.6 Discussão Comparativa . . . . . . . . . . . . . . 80 


\section{Introdução}


Seja $M^{n}$ uma variedade riemanniana de dimensão $n$ e seja $G \subset$ Iso $(M)$ um subgrupo fechado e conexo do grupo das isometrias de $M$. A ação isométrica $G \times M \rightarrow M$ é uma ação própria e, com esse significado, diremos que $M$ é uma $G$-variedade riemanniana. A ação de $G$ (ou a variedade $M)$ é dita ser de co-homogeneidade 1 se a codimensão mínima das órbitas for 1 (precisaremos melhor essa definição mais adiante).

O presente trabalho se insere no estudo das imersões isométricas de $G$ variedades riemannianas em formas espaciais. Pode-se dizer que tal estudo se iniciou com [Ko1] (1958), onde o autor mostra que uma hipersuperfície homogênea compacta do espaço euclidiano é isométrica a uma esfera. A classificação das hipersuperfícies homogêneas continua em [NaTa], [Ry], [Ha], [Ta1], [Ta2] e [TaTk] (essa relação não pretende ser completa). Descreveremos essas classificações (ao menos nos casos euclidiano e hiperbólico) no Capítulo 3.

Partindo da classificação das hipersuperfícies homogêneas de formas espaciais reais, há três caminhos naturais a se seguir: o estudo das hipersuperfícies homogêneas de formas espaciais complexas; o das subvariedades homogêneas em codimensão 2 de formas espaciais reais; e o das hipersuperfícies do cohomogeneidade 1 de formas espaciais reais.

Quanto ao primeiro caminho, já se tem a classificação das hipersuperfícies reais homogêneas do espaço projetivo complexo. Essa classificação é estudada em [Tk], [PoTh], [SuTk], [CKKT], e [KKT]. Não temos muitas informações sobre os outros casos (veja, entretanto, [Ber] para as hipersuperfícies homogêneas do hiperbólico complexo).

Com relação às subvariedades homogêneas de codimensão 2, pode-se dizer que a classificação está a bom caminho. Há os trabalhos [ACN] e [CaNo] para subvariedades do espaço euclidiano, e os autores do último continuam estudando os casos da esfera e do espaço hiperbólico.

Enfim, o caso que nos interessa mais de perto: as hipersuperfícies de co-homogeneidade 1 de formas espaciais reais. Os primeiros trabalhos que apareceram a propósito foram [PoSp] e [AMN], onde são dadas condições suficientes para que uma hipersuperfície de co-homogeneidade 1 do espaço euclidiano seja de revolução. Ambos os trabalhos consideram somente variedades compactas. Assim, em [Se], Seixas estende os resultados acima para 
variedades completas. O objetivo do presente trabalho é o de estender o resultado de [PoSp] (ou melhor, a sua extensão em [Se]) às hipersuperfícies de co-homogeneidade 1 do espaço hiperbólico. Futuramente, pretendemos obter também uma extensão de [AMN] (infelizmente não foi possível finalizá-la em tempo de apresentá-la aqui). Outros trabalhos nesse contexto são [MPS] e $[\mathrm{Fu}]$.

O trabalho está dividido em três capítulos. No primeiro, apresentamos sucintamente uma série de conceitos básicos, sem demonstrá-los, com o objetivo de fixar notações e facilitar a leitura do restante do trabalho.

O segundo capítulo concerne os aspectos da geometria hiperbólica envolvidos no problema considerado. Além de alguns conceitos e idéias já bastante conhecidos (como as subvariedades umbílicas), são consideradas também as funções distância do espaço hiperbólico e suas propriedades. Estas constituirão uma ferramenta importante no decorrer do trabalho.

O terceiro é o capítulo principal. Nele, enunciamos e demonstramos o teorema principal deste trabalho. São várias páginas cheias de lemas e proposições, organizadas, assim esperamos, de modo a propiciar uma leitura pouco tediosa.

Gostaríamos de agradecer à Prof. Maria Helena Noronha da California State University, Northridge, pela importante colaboração dada a esse trabalho. Um agradecimento especial vai ao Prof. Fabio Podestà, da Università degli Studi di Firenze, pelo apoio dado durante o estágio em Florença. 
Capítulo 1

Conceitos Básicos 
Este capítulo tem o intuito de fixar notações e apresentar algumas propriedades básicas de conceitos que serão amplamente usados no decorrer do trabalho. A matemática envolvida é bastante conhecida e, por esse motivo, não nos deteremos em detalhes nem em provas. Para maiores detalhes, citamos $[\mathrm{Da}]$ e $[\mathrm{dC}]$ para a primeira seção, [O’N] para a segunda e $[\mathrm{PaTe}],[\mathrm{Br}]$, [Ko2] e [AlAl] para a terceira.

\subsection{Imersões Isométricas}

Sejam $M^{n}$ e $\bar{M}^{m}$ variedades riemannianas com conexões de Levi-Civita $\nabla$ e $\bar{\nabla}$, respectivamente, e seja $f: M \rightarrow \bar{M}$ uma imersão isométrica. Como de costume, simplificaremos a notação identificando os espaços tangentes $T_{x} M$ e $T_{f(x)}\left(\mathrm{d} f_{x}\left(T_{x} M\right)\right)$ e os campos $X \in \mathfrak{X}(M)$ e $f_{*} X \in \mathfrak{X}(\bar{M})$, onde, lembrando, $\left(f_{*} X\right)(f(p))=\mathrm{d} f_{p}(X(p))$. Com essa identificação, e considerando a decomposição $T_{p} \bar{M}=T_{p} M \oplus T_{p} M^{\perp}$, obtemos as conhecidas fórmulas de Gauss e Weingarten:

$$
\begin{gathered}
\bar{\nabla}_{X} Y=\nabla_{X} Y+\alpha(X, Y) \forall X, Y \in \mathfrak{X}(M) \\
\bar{\nabla}_{X} \xi=-A_{\xi} X+\nabla_{X}^{\perp} \xi \quad \forall X \in \mathfrak{X}(M), \forall \xi \in \mathfrak{X}(M)^{\perp}
\end{gathered}
$$

Na primeira, $\alpha$ é a segunda forma fundamental da imersão $f$, e $A_{\xi}$, na segunda, é o relativo operador de forma (ou de Weingarten). Ainda na segunda fórmula, $\nabla^{\perp}$ é a conexão normal ao longo da imersão $f$.

Relacionado à segunda forma fundamental, há um conceito do qual se fará enorme uso durante todo o trabalho, a saber, o de número tipo:

Definição 1.1 Seja $f: M^{n} \rightarrow \bar{M}^{n+p}$ uma imersão isométrica, $x \in M$ e $\left\{\xi_{1}, \ldots, \xi_{p}\right\}$ uma base de $T_{x} M^{\perp}$. O número tipo $\tau(x)$ de $f$ em $x$ (também indicado com $\tau_{f}(x)$ quando for necessário especificar a imersão) é o número máximo de vetores $X_{1}, \ldots, X_{r}$ de $T_{x} M$ para os quais o conjunto $\left\{A_{\xi_{i}} X_{j}\right.$ । $1 \leq i \leq p, 1 \leq j \leq r\}$ é linearmente independente. 
Note que quando $M$ é uma hipersuperfície de $\bar{M}$ (isto é, $p=1$ ), o número tipo coincide com o posto da segunda forma fundamental.

Indiquemos agora com $R$ e $\bar{R}$, respectivamente, os tensores de curvatura de $M$ e $\bar{M}$, e com $R^{\perp}$ o tensor normal de curvatura relativo à imersão $f$. Para evitar ambigüidades existentes na literatura, convém explicitarmos, tomando como exemplo o tensor $R$ de $M$, que aqui usamos a seguinte definição de tensor de curvatura (em [O’N], o sinal é trocado):

$$
R(X, Y) Z=\nabla_{X} \nabla_{Y} Z-\nabla_{Y} \nabla_{X} Z-\nabla_{[X, Y]} Z .
$$

A curvatura seccional, nesse caso, é dada por

$$
K(X, Y)=\langle R(X, Y) Y, X\rangle, \quad \text { com } X, Y \text { ortonormais. }
$$

Para uma imersão isométrica $f$, a sua segunda forma fundamental e a sua conexão normal satisfazem as equações fundamentais de

\section{Gauss}

$$
\begin{aligned}
\langle R(X, Y) Z, W\rangle= & \langle\bar{R}(X, Y) Z, W\rangle+ \\
& +\langle\alpha(X, W), \alpha(Y, Z)\rangle-\langle\alpha(X, Z), \alpha(Y, W)\rangle
\end{aligned}
$$

\section{Codazzi}

$$
(\bar{R}(X, Y) Z)^{\perp}=\left(\nabla_{X}^{\perp} \alpha\right)(Y, Z)-\left(\nabla_{Y}^{\perp} \alpha\right)(X, Z)
$$

ou equivalentemente

$$
(\bar{R}(X, Y) \xi)^{\top}=\left(\nabla_{Y} A\right)(X, \xi)-\left(\nabla_{X} A\right)(Y, \xi)
$$

Ricci

$$
(\bar{R}(X, Y) \xi)^{\perp}=R^{\perp}(X, Y) \xi+\alpha\left(A_{\xi} X, Y\right)-\alpha\left(X, A_{\xi} Y\right)
$$

onde $X, Y, Z, W \in \mathfrak{X}(M), \xi \in \mathfrak{X}(M)^{\perp},(\quad)^{\top}$ e ()$^{\perp}$ são as projeções tangente e normal, e $\nabla_{X}^{\perp} \alpha$ e $\nabla_{Y} A$ são dados por

$$
\begin{gathered}
\left(\nabla_{X}^{\perp} \alpha\right)(Y, Z)=\nabla_{X}^{\perp} \alpha(Y, Z)-\alpha\left(\nabla_{X} Y, Z\right)-\alpha\left(Y, \nabla_{X} Z\right) \\
\left(\nabla_{Y} A\right)(X, \xi)=\nabla_{Y} A_{\xi} X-A_{\xi} \nabla_{Y} X-A_{\nabla_{Y}} X
\end{gathered}
$$


$\mathrm{O}$ caso que mais nos interessa é quando a variedade ambiente $\bar{M}$ tem curvatura seccional constante $c$. Nessa hipótese, as equações fundamentais tornam-se, respectivamente

\section{Gauss}

$$
\begin{aligned}
\langle R(X, Y) Z, W\rangle= & c\langle(X \wedge Y) Z, W\rangle+ \\
& +\langle\alpha(X, W), \alpha(Y, Z)\rangle-\langle\alpha(X, Z), \alpha(Y, W)\rangle
\end{aligned}
$$

\section{Codazzi}

$$
\begin{array}{lcc}
\left(\nabla_{X}^{\perp} \alpha\right)(Y, Z) & =\left(\nabla_{Y}^{\perp} \alpha\right)(X, Z) \\
& \text { ou } \\
\left(\nabla_{Y} A\right)(X, \xi) & =\left(\nabla_{X} A\right)(Y, \xi)
\end{array}
$$

Ricci

$$
\left\langle R^{\perp}(X, Y) \xi, \eta\right\rangle=\left\langle\left[A_{\xi}, A_{\eta}\right] X, Y\right\rangle .
$$

Com relação à curvatura seccional, da equação de Gauss 1.3 segue-se

$$
K(X, Y)=\bar{K}(X, Y)+\langle\alpha(X, X), \alpha(Y, Y)\rangle-\|\alpha(X, Y)\|^{2}
$$

onde $K$ e $\bar{K}$ indicam as curvaturas seccionais do plano gerado pelos campos ortonormais $X$ e $Y$ em $M$ e $\bar{M}$, respectivamente.

\subsubsection{Imersões umbílicas}

Ainda nas notações anteriores, a imersão isométrica $f$ é dita umbilica em $x \in M$ na direção $\xi \in T_{x} M^{\perp}$ se valer uma das seguintes condições (equivalentes):

1. $\exists \lambda_{\xi} \in \mathbb{R}$ tal que $A_{\xi}=\lambda_{\xi} I$

2. $A_{\xi}=\langle H(x), \xi\rangle I$

3. $\alpha(X, Y)=\langle X, Y\rangle H(x), \quad \forall X, Y \in T_{x} M$ 
onde $H(x)$ é o vetor curvatura média em $x \in M$, e $I: T_{x} M \rightarrow T_{x} M$ é a identidade.

Se uma das condições acima valer para todos os vetores $\xi \in T_{x} M^{\perp}$, dizemos que $f$ é totalmente umbílica em $x$, e se isso valer para todo $x \in M$, dizemos que $f$ é totalmente umbílica (ou simplesmente umbílica, quando não houver ambigüidade).

Da equação de Codazzi 1.8 segue-se uma primeira propriedade (Prop. 1.7 em [Da], pg. 11):

Proposição 1.2 Se $f: M^{n} \rightarrow \bar{M}_{c}^{n+p}, n \geq 2$, é uma imersão umbílica, então o vetor curvatura média é $\nabla^{\perp}$-paralelo e o tensor normal de curvatura $R^{\perp} e ́$ identicamente nulo.

Ainda mais importante aos nossos propósitos são as imersões umbílicas de hipersuperfícies de curvatura constante em espaços também de curvatura constante. Temos a seguinte proposição (cf. Prop. 5.5 em [Da], pg. 71):

Proposição 1.3 Seja $f: M_{c}^{n} \rightarrow \bar{M}_{\bar{c}}^{n+1}, n \geq 3$, uma imersão isométrica entre variedades de curvatura constante. Então

(i) $c=\bar{c}$ e $\tau \leq 1$

ou

(ii) $c>\bar{c}$ e $f$ é umbílica.

Voltaremos mais tarde a falar de imersões umbílicas, no contexto específico do espaço hiperbólico.

\subsubsection{Teoremas de rigidez}

Ao se estudar a geometria extrínseca de uma variedade a partir de sua geometria intrínseca, um conceito muito útil é o de rigidez.

Definição 1.4 Uma imersão isométrica $f: M^{n} \rightarrow \bar{M}^{m}$ é dita rígida se, dada qualquer outra imersão isométrica $g: M^{n} \rightarrow \bar{M}^{m}$, existe uma isometria $\imath$ de $\bar{M}$ tal que $g=\imath \circ f$. 
No caso de imersões isométricas em formas espaciais reais há vários teoremas de rigidez, envolvendo diferentes condições sobre a variedade. A nós interessa particularmente o seguinte teorema de Allendoerfer (cf. [Al]):

Teorema 1.5 (Allendoerfer) Seja $f: M^{n} \rightarrow Q_{c}^{n+p}$ uma imersão isométrica. Se $f$ tem número tipo $\tau \geq 3$ em todo ponto, então é rígida.

No teorema acima, $Q_{c}^{m}$ indica uma forma espacial real $m$-dimensional com curvatura seccional $c$. Usaremos essa notação em todo o trabalho.

Observação 1.6 O teorema de Allendoerfer estende o teorema de BeezKilling (cf. [Be] e [Ki]), o qual considera o caso $c=0$ e $p=1$.

\subsubsection{Homotetias}

Concluímos esta seção com algumas considerações sobre homotetias, as quais serão úteis mais tarde. Uma homotetia é um difeomorfismo conforme com fator de conformidade constante. Mais precisamente, um difeomorfismo $\phi: M^{n} \rightarrow \bar{M}^{n}$ é uma homotetia com coeficiente $k \neq 0$ se $\phi^{*}\left(g_{\bar{M}}\right)=k g_{M}$, isto é,

$$
\langle\mathrm{d} \phi(X), \mathrm{d} \phi(Y)\rangle_{\bar{M}}=k\langle X, Y\rangle_{M} \quad \forall X, Y \in T_{p} M, p \in M .
$$

Se $k>0$ (respectivamente $k<0$ ), $\phi$ é chamada de homotetia positiva (respectivamente negativa) com fator de escala $|k|^{1 / 2}$.

Por exemplo, em $\mathbb{R}^{n+1}$, a aplicação $x \longmapsto(b / a) x$ é uma homotetia positiva de $\mathbb{S}^{n}(a)$ em $\mathbb{S}^{n}(b)$ com fator de escala $b / a$. Uma isometria nada mais é que uma homotetia com $k=1$.

Uma homotetia preserva a conexão de Levi-Civita (esse fato deriva diretamente da fórmula de Koszul para conexões), e portanto preserva todas as noções geométricas que dependem unicamente da conexão, como por exemplo o transporte paralelo, geodésicas, tensor de curvatura e de Ricci.

Por outro lado, homotetias não preservam a curvatura seccional. De fato, se $\phi: M^{n} \rightarrow \bar{M}^{n}$ é uma homotetia com coeficiente $k$, então $\forall X, Y \in \mathfrak{X}(M)$

$$
K_{\bar{M}}(\mathrm{~d} \phi(X), \mathrm{d} \phi(Y))=\frac{1}{k} K_{M}(X, Y)
$$


Note, em particular, que se $k>0$, então $\phi$ preserva o sinal da curvatura. Um exemplo para nós importante de variedades homotéticas com coeficiente positivo será visto na próxima seção: são as fibras de um produto torcido. Nesse contexto, saber que uma das fibras tem curvatura seccional constante implica, pela observação acima, que todas as outras têm curvatura constante e de mesmo sinal. Isso será usado na demonstração do teorema principal.

\subsection{Produtos Torcidos}

Dadas duas variedades riemannianas $\left(B, g_{B}\right)$ e $\left(F, g_{F}\right)$, a variedade produto $B \times F$ possui uma métrica canônica dada por $g=\pi_{B}^{*} g_{B}+\pi_{F}^{*} g_{F}$, onde $\pi_{B}$ e $\pi_{F}$ são as projeções sobre $B$ e $F$. Com essa métrica, as subvariedades do tipo $B \times\{f\}$ e $\{b\} \times F$ são cópias isométricas de $B$ e $F$, respectivamente. Além disso, para todo par de campos $X \in \mathfrak{X}(B), Y \in \mathfrak{X}(F)$, tem-se que a curvatura seccional do plano por eles gerado é nula. Introduziremos agora uma outra métrica no produto $B \times F$, "torcendo", em algum sentido, a métrica de $F$ e preservando a métrica de $B$.

Sejam $B$ e $F$ variedades riemannianas e seja $\rho: B \rightarrow(0, \infty)$ uma aplicação diferenciável em $B$ e estritamente positiva. Definimos em $B \times F$ a métrica $g$ dada por

$$
g=\pi_{B}^{*} g_{B}+(\rho \circ \pi)^{2} \pi_{F}^{*} g_{F} .
$$

De outro modo, se $X$ e $Y$ são vetores tangentes a $B \times F$ em $(p, q)$, então

$$
\langle X, Y\rangle_{B \times F}=\left\langle\mathrm{d} \pi_{B}(X), \mathrm{d} \pi_{B}(Y)\right\rangle_{B}+\rho(p)^{2}\left\langle\mathrm{~d} \pi_{F}(X), \mathrm{d} \pi_{F}(Y)\right\rangle_{F} .
$$

A variedade $(B \times F, g)$ é chamada de produto torcido de $B$ e $F$ e é indicada por $B \times{ }_{\rho} F$. A função $\rho$ é chamada de fator de torção, $B$ é a base e $F$ a fibra.

Dizemos que uma variedade riemanniana $M$ tem estrutura local de produto torcido se cada ponto $x \in M$ possui uma vizinhança isométrica a um produto torcido. 
Note que se $\rho \equiv 1$, o produto torcido nada mais é que o produto riemanniano usual. E, assim como para o produto riemanniano, também a geometria do produto torcido está fortemente relacionada às geometrias da base e da fibra. Mas antes de ver essas relações, precisamos de algumas notações.

Seja $M=B \times F$ e $p=(b, f) \in M$. É natural definir os espaços

$$
T_{p} B:=\left(\mathrm{d} \pi_{F}\right)_{p}^{-1}(0)=\left\{(X, 0) \mid X \in T_{b} B\right\}
$$

$\mathrm{e}$

$$
T_{p} F:=\left(\mathrm{d} \pi_{B}\right)_{p}^{-1}(0)=\left\{(0, X) \mid X \in T_{f} F\right\} .
$$

Assim, temos a decomposição $T_{p} M=T_{p} B \oplus T_{p} F$. Observando a expressão da métrica de $M$, vê-se que essa decomposição é ortogonal. Além disso, tem-se que as cópias $B \times\{f\}$ da base $B$, chamadas folhas, são isométricas a $B$, enquanto que as cópias $\{b\} \times F$ da fibra $F$, ainda chamadas fibras, são (positivamente) homotéticas a $F$ com coeficiente $\rho(b)^{2}$ (ou fator de escala $\rho(b))$.

Ainda seguindo a nomenclatura usual, os vetores de $T_{p} B$ são chamados de vetores horizontais e os de $T_{p} F$ de verticais. Dado um campo horizontal $X \in \mathfrak{X}(B)$, o levantamento horizontal $\widetilde{X} \in \mathfrak{X}(M)$ de $X$ é o único campo tal que $\widetilde{X}_{p} \in \operatorname{ker}\left(\mathrm{d} \pi_{F}\right)_{p}$ e $\left(\mathrm{d} \pi_{B}\right)_{p}\left(\widetilde{X}_{p}\right)=X\left(\pi_{B}(p)\right) \quad \forall p \in B \times F$. De modo análogo, define-se o levantamente vertical de um campo $U \in \mathfrak{X}(F)$. Enfim, indicando com $\nabla, \nabla^{B}$ e $\nabla^{F}$, respectivamente, as conexões de Levi-Civita de $M, B$ e $F$, podemos enunciar a seguinte proposição (Prop. 35 em [O'N], pg. 206):

Proposição 1.7 Em $M=B \times_{\rho} F$, se $X, Y \in \mathfrak{X}(B)$ e $U, V \in \mathfrak{X}(F)$, então:

1. $\nabla_{\widetilde{X}} \widetilde{Y}=\widetilde{\nabla_{X}^{B} Y}$

2. $\nabla_{\tilde{X}} \widetilde{U}=\nabla_{\widetilde{U}} \widetilde{X}=(X(\rho) / \rho) \widetilde{U}$

3. $\nabla_{\widetilde{U}} \widetilde{V}=\widetilde{\nabla_{U}^{F} V}-(\langle U, V\rangle / \rho) \widehat{\operatorname{grad} \rho}$. 
Da proposição acima, segue-se o seguinte corolário:

Corolário 1.8 Nas condiçôes acima, temos que

1. as folhas $B \times\{f\}$ são totalmente geodésicas em $M$;

2. as fibras $\{b\} \times F$ são subvariedades umbílicas de $M$, com segunda forma fundamental dada por

$$
\alpha_{b}(U, V)=-\frac{\langle U, V\rangle}{\rho(b)} \widetilde{\operatorname{grad} \rho(b)} .
$$

Outra propriedade importante concerne a completude do produto torcido. Quanto a isso, tem-se o seguinte lema (Lema $40 \mathrm{em}\left[\mathrm{O}^{\prime} \mathrm{N}\right]$, pg. 209):

Lema 1.9 Se $B$ e $F$ são variedades riemannianas completas, então $B \times_{\rho} F$ é completa, qualquer que seja $\rho$.

Um típico exemplo de produto torcido são as hipersuperfícies de revolução de $\mathbb{R}^{n}$, onde a curva geratriz é a base e os paralelos são as fibras. Indicando com $g^{\prime}$ a métrica da $(n-1)$-esfera unitária, a métrica da hipersuperfície é dada por $g=\mathrm{d} t^{2}+r(t)^{2} g^{\prime}$, onde $r$ é a distãncia ao eixo de revolução.

Outro exemplo é $\mathbb{R}^{n}-\{0\}$, que pode ser escrito como $\mathbb{R}^{+} \times_{r} \mathbb{S}^{n-1}(1)$. Na verdade, basta pensar $\mathbb{R}^{n}-\{0\}$ como a hipersuperfície de revolução de $\mathbb{R}^{n+1}$ gerada pelo semi-eixo $x_{1}>0$ em torno do eixo $x_{n+1}$.

Mais tarde, ao falar de espaços hiperbólicos, veremos exemplos análogos a esses. Vejamos agora as propriedades do tensor de curvatura de um produto torcido.

\subsubsection{Tensor de curvatura de um produto torcido}

As propriedades que mais nos interessam dos produtos torcidos são aquelas que exprimem as relações entre os tensores de curvatura da base, da fibra e o da variedade produto. Essas relações são dadas pela proposição seguinte (Prop. 42 em [O'N], pg. 210), onde, para simplificar a notação, identificamos os campos horizontais e verticais com seus respectivos levantamentos: 
Proposição 1.10 Seja $M=B \times{ }_{\rho} F$ um produto torcido com tensor de curvatura $R$, e sejam $R_{B}$ e $R_{F}$ os tensores de curvatura de $B$ e $F$, respectivamente. Então, para $X, Y, Z \in \mathfrak{X}(B)$ e $U, V, W \in \mathfrak{X}(F)$, temos:

1. $R(X, Y) Z=R_{B}(X, Y) Z$

2. $R(U, X) Y=-\left(\operatorname{Hess}_{\rho}(X, Y) / \rho\right) U$

3. $R(X, Y) V=R(V, W) X=0$

4. $R(V, X) W=(\langle V, W\rangle / \rho) \nabla_{X}(\operatorname{grad} \rho)$

5. $R(V, W) U=R_{F}(V, W) U-\left(\langle\operatorname{grad} \rho, \operatorname{grad} \rho\rangle / \rho^{2}\right)(V \wedge W) U$

onde

$$
\operatorname{Hess}_{\rho}(X, Y):=X Y(\rho)-\left(\nabla_{X} Y\right)(\rho)=\left\langle\nabla_{X}(\operatorname{grad} \rho), Y\right\rangle
$$

$e$

$$
(V \wedge W) U:=\langle W, U\rangle V-\langle V, U\rangle W .
$$

Observação 1.11 É interessante observar que, pelo item 2 da proposição acima, a curvatura seccional de planos gerados por um campo horizontal e um vertical não depende deste último. De fato, dado $X \in \mathfrak{X}(B)$, então para todo $U \in \mathfrak{X}(F)$ unitário tem-se que $\langle R(U, X) X, U\rangle=-\operatorname{Hess}_{p}(X, X) / \rho$.

Finalmente, concluímos esta seção com a seguinte

Observação 1.12 Vale a pena considerar a proposição acima no caso particular em que a base é unidimensional e, mais precisamente, uma geodésica de $M$, pois usaremos isso mais adiante no trabalho. Seja $X$ um campo horizontal unitário e paralelo. Temos então:

(a) do item 5 da proposição, indicando com $K$ e $K_{F}$ as curvaturas seccionais de $M$ e $F$, respectivamente, segue-se que $K_{F}(U, V)=K(U, V)+\left(\rho^{\prime} / \rho\right)^{2}$;

(b) do item 2 , tem-se que $R(U, X) X=-\left(\rho^{\prime \prime} / \rho\right) U$, que por sua vez implica em $K(U, X)=-\rho^{\prime \prime} / \rho$;

(c) enfim, do item $4, R(X, V) W=-\langle V, W\rangle\left(\rho^{\prime \prime} / \rho\right) X$. 


\subsection{Grupos de Transformações e Ações de Co-homogeneidade 1}

Seja $M$ uma variedade diferenciável e $G$ um grupo de Lie. Uma ação diferenciável de $G$ sobre $M$ é uma aplicação diferenciável $\rho: G \times M \rightarrow M$ tal que $e(x)=x$ e $\left(g_{1} g_{2}\right)(x)=g_{1}\left(g_{2}(x)\right)$, onde $e$ é a identidade de $G$. Em outras palavras, $\rho$ define um homomorfismo de $G$ no grupo dos difeomeorfismos de $M$. Quando se há uma ação desse tipo, diz-se também que $M$ é uma $G$-variedade.

Para uma $G$-variedade $M$ e para cada ponto $x \in M$, definem-se a órbita de $x$ e a isotropia de $G$ em $x$ por, respectivamente:

$$
\begin{gathered}
G(x):=\{g(x) \mid g \in G\} \\
G_{x}:=\{g \in G \mid g(x)=x\} .
\end{gathered}
$$

Dado $x \in M$, a aplicação orbital $\omega_{x}: G \rightarrow M$ definida por $\omega_{x}(g)=g(x)$ é constante nas classes laterais da isotropia $G_{x}$, e portanto passa ao quociente. A aplicação quociente $\bar{\omega}_{x}: G / G_{x} \rightarrow M$ é injetora e tem como imagem a órbita $G(x)$. Esta, portanto, é uma variedade diferenciável com a estrutura diferenciável induzida pela variedade quociente $G / G_{x}$.

Proposição 1.13 Seja $M$ uma $G$-variedade. Então, para todo $x, y \in M$

1. $G_{g(x)}=g G_{x} g^{-1}$

2. se $G(x) \cap G(y) \neq \varnothing$, então $G(x)=G(y)$.

Indicaremos com $M / G$ o conjunto das órbitas e com $\pi: M \rightarrow M / G$ a projeção $x \longmapsto G(x)$. Com a topologia quociente, $M / G$ é chamado espaço de órbitas.

Se $H$ é um subgrupo de $G$, denotamos por $(H)$ a classe de conjugação de $H$. Se $G(x)$ é uma órbita de $M$, o conjunto dos subgrupos de isotropia $G_{g(x)}=g G_{x} g^{-1}$ constitui a classe de conjugação $\left(G_{x}\right)$, chamada tipo de isotropia da órbita $G(x)$. 
Uma aplicação $f: M \rightarrow N$ entre duas $G$-variedades é dita equivariante se $f(g(x))=g(f(x))$, para todo $(g, x) \in G \times M$. Nesse caso, tem-se que $f(G(x))=G(f(x))$ e $G_{x} \subseteq G_{f(x)}$, onde a igualdade vale se e somente se $f$ é um difeomorfismo sobre $G(f(x))$. Segue-se, portanto, que duas órbitas têm o mesmo tipo de isotropia se e somente se são equivariantemente difeomorfas.

Uma órbita $G(x)$ é chamada órbita principal (e $\left(G_{x}\right)$ de tipo de isotropia principal) se existe uma vizinhança $U$ de $x$ tal que para todo $y \in U$ existe uma aplicação equivariante de $G(x)$ em $G(y)$ (ou, equivalentemente, se existe $g \in G$ tal que $\left.G_{x} \subseteq g G_{y} g^{-1}\right)$.

Um ponto $x \in M$ é dito regular se a órbita $G(x)$ for principal, e o conjunto dos pontos regulares será indicado com $M_{\text {reg }}$. Como visto acima, as órbitas principais são equivariantemente difeomorfas entre si. Em particular, têm a mesma dimensão. A ação $G \times M \rightarrow M$ é dita ser de co-homogeneidade $k$ se a codimensão das órbitas principais em $M$ for $k$.

Os pontos cujas órbitas não são principais são chamados singulares. Dentre as órbitas (ou pontos) singulares, distinguimos ainda as chamadas excepcionais que são aquelas que têm a mesma dimensão das órbitas principais.

Uma subvariedade $S$ de $M$ é chamada uma fatia em $x \in M$, se existe uma vizinhança $G$-invariante $U$ de $x$ e uma retração equivariante $r: U \rightarrow G(x)$ tal que $S=r^{-1}(x)$.

Proposição 1.14 Na notação acima, temos:

1. $x \in S$ e $G_{x}(S) \subseteq S$;

2. se $g(S) \cap S \neq \varnothing$, então $g \in G_{x}$;

3. $G(S)=\{g(s) \mid(g, s) \in G \times S\}$ é aberto em $M$.

Corolário 1.15 Se S é uma fatia em $x$, então:

1. S é uma $G_{x}$-variedade;

2. se $y \in S$, então $G_{y} \subseteq G_{x}$;

3. se $G(x)$ é uma órbita principal e $G_{x}$ é compacto, então $G_{y}=G_{x}$ para todo $y \in S$; 
4. duas $G_{x}$-órbitas $G_{x}\left(s_{1}\right)$ e $G_{x}\left(s_{2}\right)$ são do mesmo tipo, se e somente se as órbitas $G\left(s_{1}\right)$ e $G\left(s_{2}\right)$ são do mesmo tipo;

5. $S / G_{x}=G(S) / G$, e é uma vizinhança aberta de $G(x)$ em $M / G$.

Proposição 1.16 Se $x \in M$ é regular e $S$ é uma fatia em $x$, então a aplicação $(g(x), s) \longmapsto g(s)$ define um difeomorfismo entre $G(x) \times S$ e $G(S)$.

As ações de grupos que nos interessam são as ações isométricas, ou seja, aquelas onde o grupo age por isometrias sobre a variedade. Nesse contexto, é particularmente importante o seguinte conceito:

Definição 1.17 Uma ação $G \times M \rightarrow M$ é dita própria se $g_{n} x_{n} \rightarrow y$ e $x_{n} \rightarrow x$ implicam que $\left\{g_{n}\right\}$ tem uma subseqüência convergente.

Cada uma das condições abaixo é equivalente à ação ser própria:

1. a aplicação de $G \times M \rightarrow M \times M$ dada por $(g, x) \longmapsto(g(x), x)$ é própria;

2. dados subconjuntos compactos $K, L \subset M$, então é compacto o conjunto $\{g \in G \mid g K \cap L \neq \varnothing\}$.

Observação 1.18 Se o grupo $G$ for compacto, então a ação é claramente própria. Além disso, se a ação é própria, os grupos de isotropia são compactos.

A relação das ações isométricas com as ações próprias é esclarecida a seguir.

Proposição 1.19 Seja $M$ uma $G$-variedade riemanniana. Se $G$ é um subgrupo fechado do grupo das isometrias de $M$, então a ação é própria.

Teorema 1.20 Se $G \times M \rightarrow M$ é uma ação própria, então existe uma métrica $G$-invariante sobre $M$, isto é, a ação de $G$ sobre $M$ é isométrica em relação a essa métrica.

Em outras palavras, ao se falar em ação própria, podemos sempre supor que o grupo em questão é um subgrupo fechado do grupo das isometrias de $M$ com relação a uma oportuna métrica. E é o que faremos de agora em diante: $M$ será uma variedade riemanniana sobre a qual age propriamente 
um subgrupo fechado $G$ do seu grupo de isometrias (ou mais sucintamente, diremos que $M$ é uma $G$-variedade riemanniana).

Algumas boas propriedades passam a valer no caso de ações próprias:

Propriedade 1.21 Se $M$ é uma G-variedade riemanniana, então:

1. as órbitas são subvariedades fechadas e mergulhadas em $M$;

2. para todo ponto $x \in M$, existe uma fatia em $x$;

3. o conjunto $M_{\text {reg }}$ dos pontos regulares é aberto e denso em $M$;

4. para cada $g \in G, \operatorname{Fix}(g)=\{x \in M \mid g(x)=x\}$ é uma subvariedade totalmente geodésica de $M$.

Quanto à existência de fatias, podemos ser mais explícitos. Dado $x \in M$, seja $y \in G(x)$ qualquer e sejam

$$
T_{y}^{r} G(x)^{\perp}:=\left\{u \in T_{y} G(x)^{\perp} \mid\|u\| \leq r\right\}
$$

$\mathrm{e}$

$$
T^{r} G(x)^{\perp}:=\left\{u \in T_{y} G(x)^{\perp} \mid y \in G(x),\|u\| \leq r\right\} .
$$

Se $r>0$ é tal que $\exp \left(T^{r} G(x)^{\perp}\right)$ é uma vizinhança tubular de $G(x)$, então o conjunto $S_{x}=\exp _{x}\left(T_{x}^{r} G(x)^{\perp}\right)$ é tal que:

1. $S_{g(x)}=g S_{x}$

2. $S_{x}$ é uma fatia em $x$, chamada fatia normal em $x$.

Seja agora $M$ uma $G$-variedade riemanniana e $N$ uma órbita de $M$. Um campo normal $\xi \in T N^{\perp}$ é dito equivariante se $\mathrm{d} g_{x}(\xi(x))=\xi(g(x))$ para todo $g \in G$ e $x \in N$.

Em particular, se $x \in M$ é um ponto regular, e $\xi_{x} \in T_{x} G(x)^{\perp}$, o campo $\hat{\xi}:=\mathrm{d} g_{x}\left(\xi_{x}\right)$ está bem definido e é um campo normal equivariante. Mais ainda, se $\left\{\xi_{x}^{i}\right\}$ é uma base de $T_{x} G(x)^{\perp}$, então $\left\{\hat{\xi}^{i}\right\}$ é um referencial ortonormal global equivariante ao longo de $G(x)$ (em outras palavras, o fibrado normal de $G(x)$ é trivial). 
Proposição 1.22 Seja $M$ uma G-variedade riemanniana, $N$ uma órbita de $M$, e $\xi$ um campo normal equivariante em $N$. Então:

1. $A_{\xi(g(x))}=\mathrm{d} g_{x} \circ A_{\xi(x)} \circ \mathrm{d} g_{x}^{-1}$, para todo $x \in N$, onde $A_{\xi}$ é o operador de Weingarten da imersão $N \hookrightarrow M$ na direção $\xi$;

2. as curvaturas principais de $N$ na direção $\xi$ são constantes;

3. $\{\exp (\xi(x)) \mid x \in N\}$ é uma G-órbita.

Teorema 1.23 Seja $M$ uma $G$-variedade riemanniana e seja $M_{\text {sing }}$ o conjunto dos pontos singulares de $M$. Então $M_{\text {sing }} / G$ não desconecta $M / G$.

Corolário 1.24 Se $M$ é conexa, então:

1. $M / G$ é conexa;

2. $M$ tem um único tipo de órbita principal.

Para completar essas considerações de caráter geral das ações isométricas, antes de entrar no específico das ações de co-homogeneidade 1, é necessário falar, mesmo que "tangencialmente", de um dos conceitos centrais da teoria das ações de grupos, o de seção.

Seja $M$ uma $G$-variedade riemanniana. Uma seção é uma subvariedade fechada, conexa e mergulhada de $M$ que encontra todas as órbitas ortogonalmente.

Em geral, ações isométricas quaisquer não admitem seções (aquelas que as admitem são chamadas de polares). Não é do nosso interesse aprofundar esse assunto, mas, por completeza de informação, vale citar a Proposição 5.6.9, pg. 96, de [PaTe] (que é, na verdade, um teorema de existência de seções). Para nós é suficiente considerar a seguinte proposição (por sinal, intimamente ligada àquela citada):

Proposição 1.25 Seja $M$ uma $G$-variedade riemanniana conexa e completa. Para cada ponto regular $x \in M$ defina $\mathcal{T}(x)$ como sendo a imagem de $T_{x} G(x)^{\perp}$ pela aplicação exponencial, e $\mathcal{T}_{r}(x)=\mathcal{T}(x) \cap M_{\text {reg. }}$. Então:

1. $g \mathcal{T}(x)=\mathcal{T}(g(x))$ e $g \mathcal{T}_{r}(x)=\mathcal{T}_{r}(g(x))$; 
2. $\forall \xi \in T_{x} G(x)^{\perp}$ a geodésica $\exp _{x}(t \xi)$ é ortogonal a toda órbita que encontra;

3. $\mathcal{T}(x)$ encontra toda órbita de $M$.

Observação 1.26 A proposição acima é uma adaptação da Proposição 5.6.2 de [PaTe], pg. 94. Nesta, o item 3 acima é enunciado para $G$ compacto. A compacidade do grupo serve unicamente para afirmar o seguinte: dado um ponto $y \in M$, existe $g \in G$ tal que $g(y)$ minimiza a distância de $x$ a $G(y)$. Mas, sendo $M$ completa e conexa, basta termos $G$ fechado para poder fazer tal afirmação (obtemos um compacto intersecando a órbita de $y$ com uma oportuna bola fechada centrada em $x$ ).

\subsubsection{Ações de co-homogeneidade 1}

A partir de agora nos restringiremos às ações de co-homogeneidade 1 . Neste caso, o espaço de órbitas é uma variedade unidimensional, e portanto homeomorfa a $\mathbb{R}, \mathbb{S}^{1}, \mathbb{R}^{+}$ou $[0,1]$. Além do mais, nos dois primeiros casos, todas as órbitas são regulares, enquanto que nos dois últimos as órbitas singulares correspondem aos pontos do bordo.

Seja dada uma variedade riemanniana conexa e completa $M$ e seja $G$ um subgrupo fechado e conexo de Iso $(M)$ que age com co-homogeneidade 1 sobre $M$.

Uma geodésica normal é uma geodésica $\sigma:(a, b) \rightarrow M$ parametrizada por comprimento de arco e tal que $\sigma^{\prime}(t)$ é ortogonal à órbita $G(\sigma(t))$ em $\sigma(t)$. A Proposição 1.25 garante a existência de geodésicas normais (as quais, por ser $M$ completa, estão definidas em toda a reta, e constituem, portanto, as seções da ação de $G$ ). Juntando esse fato com as considerações já feitas sobre fatias e sobre campos equivariantes, temos, em resumo, a seguinte

Proposição 1.27 Seja $M$ uma G-variedade riemanniana completa e conexa de co-homogeneidade 1, e seja $\sigma: \mathbb{R} \rightarrow M$ uma geodésica normal. Então:

1. $\forall g \in G, \sigma_{g}:=g \circ \sigma$ é uma geodésica normal;

2. se $p=\sigma(0) \in M_{\text {reg }}$, então existe $\epsilon>0$ tal que $\sigma(-\epsilon, \epsilon)$ é uma fatia em $p$; 
3. se $S=\sigma(-\epsilon, \epsilon)$ é uma fatia em $p \in M_{\text {reg, }}$ então:

(a) $G_{\sigma(t)}=G_{p}$, para todo $t \in(-\epsilon, \epsilon)$

(b) a aplicação $\Phi:(-\epsilon, \bar{\epsilon}) \times G(p) \rightarrow G(S)$, dada por $\Phi(t, g(p))=$ $g(\sigma(t))$, é um difeomorfismo

(c) está bem definido o campo normal $\eta \in \mathfrak{X}(G(S))$, unitário e equivariante, dado por

$$
\eta(g(\sigma(t)))=\mathrm{d} g_{\sigma(t)}\left(\sigma^{\prime}(t)\right)
$$

4. $M=G(\sigma(\mathbb{R}))$.

E também, ainda com a mesma notação, temos (Prop. 4.1 item (6) de [AlAl], pgs. 199-200):

Proposição 1.28 Se $x=\sigma(t)$ é um ponto singular, então $G_{p} \subset G_{x}$ e $G_{x} / G_{p}$ é difeomorfo a uma esfera $\mathbb{S}^{m}, m \geq 0$.

Para finalizar, enunciamos mais algumas propriedades das ações de cohomogeneidade 1 .

Proposição 1.29 Seja $M$ de co-homogeneidade 1 ef $: M^{n \geq 3} \rightarrow \bar{M}_{c}^{n+1}$ uma imersấo isométrica em uma variedade de curvatura constante c. Se $x \in M_{\text {reg }}$, então:

1. se $\tau(x) \geq 2$, então $\tau$ é constante ao longo da órbita de $x$;

2. se $\tau(x) \leq 1$, então $\tau(y) \leq 1$ para todo y na órbita de $x$.

Observação 1.30 Essa proposição está demonstrada em [AMN] (Prop. 3.2, pgs 203-204) para o caso $\bar{M}_{c}=\mathbb{R}^{n+1}$, mas a prova para o caso geral é praticamente a mesma, bastando tomar (veja a demonstração) o tensor $\mathcal{R}$ apropriado, ou seja, aquele definido por

$$
\langle\mathcal{R}(X \wedge Y), Z \wedge W\rangle=\langle R(X, Y) Z-c(X \wedge Y) Z, W\rangle .
$$

Proposição 1.31 Se $M=M_{\text {reg }}$, então $M$ é difeomorfa ao produto $\Sigma \times M / G$, onde $\Sigma$ é uma qualquer órbita principal. 
Observação 1.32 A proposição acima encontra-se demonstrada em [Mo] para o caso do grupo ser compacto. Mas a proposição continua a valer no caso em questão, uma vez que a compacidade do grupo serve somente a garantir a existência de seções.

Enfim, pelo Teorema 7.4, pg 30, de [St] e pela Proposição 1.28, temos o seguinte

Teorema 1.33 Seja $\Sigma$ uma órbita principal com isotropia $H$, e $\chi$ uma órbita singular com isotropia $K \supset H$. Então $\Sigma \rightarrow \chi$ é uma fibração localmente trivial com fibra típica $K / H$, e esta é difeomorfa a uma esfera. Em particular, se $\chi$ é excepcional, a fibração acima é um recobrimento duplo. 
Capítulo 2

Geometria Hiperbólica 
Este capítulo tem por objetivo apresentar aqueles conceitos da geometria hiperbólica (e outros a esses correlatos) que de fato servem aos nossos propósitos. As principais referências usadas aqui são [O'N] e [Ra] para as primeiras duas seções, [Ce] e [CeRy] para a terceira seção, [dCDa] e [Suz] para a quarta ([Suz] é valida também para segunda seção) e [Nö] para a quinta e última seção.

\subsection{Espaço de Lorentz}

O espaço de Lorentz é o espaço linear euclidiano dotado de uma métrica não-degenerada de índice 1. Mais especificamente, dados $x, y \in \mathbb{R}^{m}$, definimos o produto interno lorentziano

$$
\langle\langle x, y\rangle\rangle:=x_{1} y_{1}+\cdots+x_{m-1} y_{m-1}-x_{m} y_{m}
$$

onde $x=\left(x_{1}, \ldots, x_{m}\right), y=\left(y_{1}, \ldots, y_{m}\right)$. O espaço linear $\mathbb{R}^{m}$, com a métrica $\left\langle\langle\right.$,$\rangle , é chamado de espaço de Lorentz e é indicado por \mathbb{L}^{m}$. Também é comum a notação $\mathbb{R}^{m-1,1}$, a qual deixa mais explícita a assinatura da métrica.

O produto interno lorentziano induz uma norma lorentziana assim como uma distância lorentziana dadas por, respectivamente:

$$
\begin{gathered}
\|x\|_{\mathbb{L}}:=\langle\langle x, x\rangle\rangle^{1 / 2} \\
d_{\mathbb{L}}(x, y):=\|x-y\|_{\mathbb{L}} .
\end{gathered}
$$

Note que a norma acima assume valores positivos, nulos ou imaginários. Um vetor $x \in \mathbb{L}^{m}$ é dito:

- tipo espaço (ou riemanniano) se $\|x\|_{\mathbb{L}}$ é real positivo;

- tipo tempo (ou lorentziano) se $\|x\|_{\mathbb{L}}$ é imaginário;

- tipo luz (ou degenerado) se $\|x\|_{\mathbb{L}}=0$.

A distinção acima é chamada de caráter causal do vetor. Além disso, um vetor $x$ do tipo tempo ou luz é dito positivo (respec. negativo) se $x_{m}>0$ 
(respec. $x_{m}<0$ ). Os vetores tipo luz $x \neq 0$ também são chamados vetores nulos. O conjunto dos vetores tipo luz, isto é, o hipercone

$$
\mathbb{V}^{m-1}=\left\{x \in \mathbb{L}^{m} \mid x_{m}^{2}=x_{1}^{2}+\cdots+x_{m-1}^{2}\right\}
$$

é chamado de cone de luz. O exterior de $\mathbb{V}^{m-1}$ é o aberto de $\mathbb{L}^{m}$ constituído pelos vetores tipo espaço, enquanto que o interior de $\mathbb{V}^{m-1}$ é o aberto (com duas componentes conexas) de $\mathbb{L}^{m}$ constituído pelos vetores tipo tempo.

Uma aplicação linear $\phi: \mathbb{L}^{m} \rightarrow \mathbb{L}^{m}$ é uma transformação de Lorentz se

$$
\langle\langle\phi(x), \phi(y)\rangle\rangle=\langle\langle x, y\rangle\rangle \quad \forall x, y \in \mathbb{L}^{m} .
$$

Uma base $\left\{x_{1}, \ldots, x_{m}\right\}$ é pseudo-ortonormal se

$$
\left\langle\left\langle x_{i}, x_{j}\right\rangle\right\rangle= \begin{cases}-1 & \text { se } i=j=m \\ \delta_{i j} & \text { se } i \neq m \text { ou } j \neq m\end{cases}
$$

A base canônica é pseudo-ortonormal. Uma aplicação $\phi: \mathbb{L}^{m} \rightarrow \mathbb{L}^{m}$ é uma transformação lorentziana se e somente se manda bases pseudo-ortonormais em bases pseudo-ortonormais.

Uma matriz lorentziana é uma matriz cuja aplicação associada é lorentziana. Temos a seguinte

Proposição 2.1 Seja A uma matriz real $m \times m$. São condições equivalentes:

1. A é lorentziana;

2. as colunas de A formam uma base pseudo-ortonormal de $\mathbb{L}^{m}$;

3. as linhas de A formam uma base pseudo-ortonormal de $\mathbb{L}^{m}$;

4. A satisfaz a equação $A^{t} J A=J$, onde

$$
J=\left(\begin{array}{cccc}
1 & 0 & & 0 \\
0 & 1 & & 0 \\
& & \ddots & \\
0 & 0 & & -1
\end{array}\right) ;
$$

5. A satisfaz a equação $A J A^{t}=J$. 
O grupo das aplicações lorentzianas de $\mathbb{L}^{m}$ (ou de suas matrizes associadas) é chamado grupo de Lorentz e indicado com $O(1, m-1)$. As aplicações lorentzianas têm determinante 1 ou -1 , e podem preservar ou não os vetores tipo tempo positivos (claramente, as aplicações de Lorentz preservam o caráter causal dos vetores). Indicaremos com $S O(1, m-1)$ o subgrupo de $O(1, m-1)$ dado pelas aplicações lorentzianas de determinante 1 (este subgrupo tem duas componentes conexas), e com $S O^{+}(1, m-1)$ o subgrupo (conexo) de $S O(1, m-1)$ dado pelas aplicações lorentzianas de determinante 1 que preservam a positividade dos vetores tipo tempo.

Um subespaço $W \subset \mathbb{L}^{m}$ é dito

- tipo tempo (ou lorentziano) se $W$ contém algum vetor tipo tempo;

- tipo espaço (ou riemanniano) se todo vetor $x \neq 0$ de $W$ é tipo espaço;

- tipo luz (ou degenerado) nos outros casos.

Em outras palavras, $W$ é lorentziano, riemanniano ou degenerado se a restrição da métrica $\langle\langle\rangle$,$\rangle de \mathbb{L}^{m}$ a $W$ for, respectivamente, não-degenerada de índice 1, positiva-definida ou degenerada.

Considerando a ortogonalidade induzida pelo produto interno lorentziano, fixado um subespaço $W \subset \mathbb{L}^{m}$ e indicando com $W^{\perp}$ o seu complemento ortogonal, temos as seguintes propriedades:

1. $W$ é lorentziano se e somente se $W^{\perp}$ é riemanniano.

2. se $\operatorname{dim} W \geq 2, W$ é lorentziano se e somente se contém dois vetores nulos linearmente independentes.

3. $\operatorname{dim} W+\operatorname{dim} W^{\perp}=m$.

4. se $W$ não é degenerado, então $\mathbb{L}^{m}=W \oplus W^{\perp}$.

5. se $W$ é degenerado, então $\operatorname{dim} W \cap W^{\perp}=1$

Veremos em seguida algumas propriedades que serão úteis mais adiante neste trabalho. 
Propriedade 2.2 Seja $W \subset \mathbb{L}^{m}$ não-degenerado. Dado $x \in \mathbb{L}^{m}$, temos $x=x_{W}+x_{W^{\perp}}$, com $x_{W} \in W$ e $x_{W^{\perp}} \in W^{\perp}$. Resulta então:

- $(x+W) \cap W^{\perp}=\left\{x_{W^{\perp}}\right\}$

- $(x+W) \cap\left(y+W^{\perp}\right)=\left\{y_{W}+x_{W^{\perp}}\right\}$

Demonstração:

$z \in x+W \Rightarrow x-z \in W \Rightarrow x_{W^{\perp}}-z_{W^{\perp}} \in W \cap W^{\perp} \Rightarrow x_{W^{\perp}}=z_{W^{\perp}}$

$z \in y+W^{\perp} \Rightarrow y-z \in W^{\perp} \Rightarrow y_{W}-z_{W} \in W \cap W^{\perp} \Rightarrow y_{W}=z_{W}$;

$\therefore z \in(x+W) \cap\left(y+W^{\perp}\right) \Rightarrow z=y_{W}+x_{W^{\perp}}$.

Propriedade 2.3 Seja $W \subset \mathbb{L}^{m}$ degenerado. Então:

- se $x \notin W+W^{\perp}$, então $(x+W) \cap W^{\perp}=\varnothing=W \cap\left(x+W^{\perp}\right)$

- se $x, y \in W+W^{\perp}$, digamos $x=x_{W}+x_{W^{\perp}}$ e $y=y_{W}+y_{W^{\perp}}$, então $(x+W) \cap\left(y+W^{\perp}\right)=\left(y_{W}+x_{W^{\perp}}\right)+W \cap W^{\perp}$

Demonstração:

É similar à da propriedade anterior.

Seja agora $G$ um subgrupo de Lie conexo de $S O^{+}(1, m-1)$, e seja Fix $G$ o subespaço de $\mathbb{L}^{m}$ constituído pelos pontos fixos de $G$.

Propriedade 2.4 Se $V \subset$ Fix $G$ é um subespaço de $\mathbb{L}^{m}$, então os subespaços afins paralelos a $V^{\perp}$ são $G$-invariantes.

\section{Demonstração:}

Como $G \subset O(1, n+1)$ e $G(V)=V$, então $G\left(V^{\perp}\right)=V^{\perp}$. Se $V$ é nãodegenerado, então $\mathbb{L}^{m}=V \oplus V^{\perp}$. Logo, $\forall x \in \mathbb{L}^{m}, x=x_{V}+x_{V^{\perp}}$ e temos

$$
x+V^{\perp}=x_{V}+V^{\perp} \text {. }
$$

Dado, então, $g \in G$ resulta

$$
g\left(x+V^{\perp}\right)=g\left(x_{V}+V^{\perp}\right)=x_{V}+V^{\perp}
$$


ou seja, $g\left(x+V^{\perp}\right)=x+V^{\perp}$. Se $V$ é degenerado, então $\operatorname{dim}\left(V+V^{\perp}\right)=m-1$ e $V \cap V^{\perp}=\mathbb{R} w$ para um certo $w \in \mathbb{L}^{m}$. Temos, nesse caso,

$$
\mathbb{L}^{m}=\operatorname{span}\left\{u, v_{1}, \ldots, v_{k}, \bar{v}_{1}, \ldots, \bar{v}_{h}, w\right\},
$$

onde $u \notin V+V^{\perp}, \operatorname{span}\left\{v_{1}, \ldots, v_{k}, w\right\}=V$ e $\operatorname{span}\left\{\bar{v}_{1}, \ldots, \bar{v}_{h}, w\right\}=V^{\perp}$. Logo, dado $x \in \mathbb{L}^{m}$ e $g \in G$, temos

$$
\begin{gathered}
x=x_{u} u+\sum x_{i} v_{i}+\sum \bar{x}_{j} \bar{v}_{j}+x_{w} w \\
x+V^{\perp}=\left(x_{u} u+\sum x_{i} v_{i}\right)+V^{\perp} \\
g\left(x+V^{\perp}\right)=\left(x_{u} g(u)+\sum x_{i} v_{i}\right)+V^{\perp} .
\end{gathered}
$$

Assim,

$$
\begin{gathered}
g\left(x+V^{\perp}\right)=x+V^{\perp} \Leftrightarrow\left\{\left(x_{u} g(u)+\sum x_{i} v_{i}\right)-\left(x_{u} u+\sum x_{i} v_{i}\right)\right\} \in V^{\perp} \\
\Leftrightarrow g(u)-u \in V^{\perp} .
\end{gathered}
$$

Mas, $\forall y \in V, \forall g \in G$,

$$
\langle g(u)-u, y\rangle=\langle g(u), y\rangle-\langle u, y\rangle=\langle g(u), g(y)\rangle-\langle u, y\rangle=0 .
$$

Portanto $g(u)-u \in V$.

Propriedade 2.5 Seja $W \subset \mathbb{L}^{m}$ um subespaço linear e $\bar{W}=p+W$ um subespaço afim paralelo a $W$. Se $G(\bar{W})=\bar{W}$ então $G(W)=W$.

\section{Demonstração:}

Para todo $g \in G$ e $v \in W$, se $g(p+v) \in \bar{W}, \exists w \in W$ tal que $g(p+v)=p+w$. Por outro lado, como $p \in \bar{W}$, existe $w^{\prime} \in W$ tal que $g(p)=p+w^{\prime}$. Assim,

$$
\begin{gathered}
p+w=g(p+v)=g(p)+g(v)=\left(p+w^{\prime}\right)+g(v) \\
\therefore g(v)=w-w^{\prime} \in W .
\end{gathered}
$$




\subsection{Espaço Hiperbólico}

Uma variedade riemanniana $n$-dimensional completa e simplesmente conexa de curvatura seccional constante $c<0$ é chamada de espaço hiperbólico (de curvatura c) e é indicada com $\mathbb{H}^{n}(c)$. Dentre os vários modelos do espaço hiperbólico, usaremos aqui o seguinte:

$$
\mathbb{H}^{n}(c)=\left\{x \in \mathbb{L}^{n+1} \mid\langle\langle x, x\rangle\rangle=1 / c, x_{n+1}>0\right\},
$$

onde $\mathbb{L}^{n+1}$ tem a assinatura de $\mathbb{R}^{n, 1}$.

Além disso, por não estarmos interessados nos diferentes valores de curvatura, assumiremos $c=-1$ (a menos de menção explícita em contrário), e indicaremos $\mathbb{H}^{n}(-1)$ simplesmente com $\mathbb{H}^{n}$.

O espaço tangente em $x \in \mathbb{H}^{n}$ é ortogonal ao vetor posição $\eta(x):=x$ e, portanto, é do tipo espaço. Logo, $\mathbb{H}^{n}$ é uma hipersuperfície riemanniana do espaço de Lorentz. Indicando com $D$ e $\nabla$, respectivamente, as conexões de Levi-Civita de $\mathbb{L}^{n+1}$ e $\mathbb{H}^{n}$, temos a fórmula de Gauss da imersão $\mathbb{H}^{n} \hookrightarrow \mathbb{L}^{n+1}$ :

$$
D_{X} Y=\nabla_{X} Y+\langle X, Y\rangle \eta, \quad \forall X, Y \in \mathfrak{X}\left(\mathbb{H}^{n}\right)
$$

onde $\langle$,$\rangle é a métrica riemanniana de \mathbb{H}^{n}$ induzida pela métrica lorentziana $\langle\langle$,$\rangle .$

As isometrias do espaço hiperbólico são as restrições a $\mathbb{H}^{n}$ das transformações lorentzianas que preservam a positividade dos vetores tipo tempo. Em símbolos, Iso $\mathbb{H}^{n}=O^{+}(1, n)$. A componente conexa da identidade é $\mathrm{SO}^{+}(1, n)$.

As geodésicas unitárias $\alpha: I \rightarrow \mathbb{H}^{n}$ do espaço hiperbólico são soluções da equação

$$
\alpha^{\prime \prime}(t)-\alpha(t)=0
$$

ou seja, são dadas por

$$
\alpha(t)=(\cosh t) p+(\operatorname{senh} t) X_{p},
$$

onde $p=\alpha(0)$ e $X_{p}=\alpha^{\prime}(0) \in T_{p} \mathbb{H}^{n}$ são as condições iniciais. 
Note que $\alpha(I)$ está totalmente contida no subespaço de $\mathbb{L}^{n+1}$ gerado por $p$ e $X$. Em outras palavras, as geodésicas de $\mathbb{H}^{n}$ são as curvas resultantes de interseç̧ões de subespaços bidimensionais de $\mathbb{L}^{n+1}$ com $\mathbb{H}^{n}$.

Dessa observação segue-se também que as subvariedades $k$-dimensionais totalmente geodésicas de $\mathbb{H}^{n}$ são obtidas por intersecções de $\mathbb{H}^{n}$ com subespaços $(k+1)$-dimensionais de $\mathbb{L}^{n+1}$.

\subsubsection{Subvariedades umbílicas de $\mathbb{H}^{n}$}

Veremos primeiramente um teorema que caracteriza as subvariedades umbílicas do espaço hiperbólico como intersecções de $\mathbb{H}^{n}$ com oportunos subespaços afins de $\mathbb{L}^{n+1}$. Em seguida, em função do caráter causal desses subespaços, descreveremos os três tipos de umbílicas, já largamente conhecidas: as esferas geodésicas, as equidistantes e as horoesferas.

Teorema 2.6 Seja $N^{k}$ uma subvariedade umbilica conexa de $\mathbb{H}^{n}$. Dado $x \in$ $N$, seja $W(x):=T_{x} N \oplus \operatorname{span}\{\widetilde{H}(x)\} \subset \mathbb{L}^{n+1}$, onde $\widetilde{H}$ é o vetor curvatura média da imersão de $N^{k}$ em $\mathbb{L}^{n+1}$. Então, $N^{k}$ está contida em $(x+W(x)) \cap$ $\mathbb{H}^{n}$.

Demonstração:

Seja $H$ o vetor curvatura média da inclusão $N^{k} \hookrightarrow \mathbb{H}^{n}$, e seja $x \in N$ tal que $H(x) \neq 0$. Sendo $N$ umbílica em $\mathbb{H}^{n}$, tem-se que $H$ é paralelo e $R^{\perp}=0$. Logo, numa vizinhança $U$ de $x$ existem campos normais paralelos $\xi_{1}, \ldots, \xi_{s} \in$ $\mathfrak{X}\left(\mathbb{H}^{n}\right), s=n-k-1$, tais que

$$
T_{y} N^{\perp}=\operatorname{span}\left\{H(y), \xi_{1}(y), \ldots, \xi_{s}(y)\right\}, \forall y \in U .
$$

Pela fórmula de Gauss 2.1, considerando extensões locais de $H$ e dos $\xi_{i}$ 's em $\mathbb{L}^{n+1}$, temos $\forall X \in \mathfrak{X}(N)$ :

i. $D_{X} \xi_{i}=\nabla_{X} \xi_{i}=0 \quad \forall i=1, \ldots, s$

ii. $D_{X} H=\nabla_{X} H=-\|H\|^{2} X$

iii. $D_{X} \eta=X$ 
De (i) segue-se que $\xi_{i}$ é constante em $\mathbb{L}^{n+1}$ ao longo de $U, \forall i=1, \ldots, s$. Defina agora $Z:=\lambda \eta+H$, onde $\lambda=\|H\|^{2}$. Pelos itens (ii) e (iii), $D_{X} Z=0$. Logo, $Z$ também é um campo constante em $\mathbb{L}^{n+1}$ ao longo de $U$.

Assim, o subespaço $V^{\perp}=\operatorname{span}\left\{Z, \xi_{1}, \ldots, \xi_{s}\right\}$ é constante em $\mathbb{L}^{n+1}$ ao longo de $U$. Por outro lado, temos:

- $T_{y} N \subset V, \quad \forall y \in U$;

- $\langle\eta+H, \zeta\rangle=0, \quad \forall \zeta \in V^{\perp}$;

- $\eta+H=\widetilde{H}$ é a curvatura média de $N^{k} \hookrightarrow \mathbb{L}^{n+1}$.

Esta última decorre de

$$
\widetilde{H}=\frac{1}{k}\left\{\left(\operatorname{tr} A_{\hat{H}}\right) \hat{H}-\left(\operatorname{tr} A_{\eta}\right) \eta\right\},
$$

onde $\hat{H}=H /\|H\|$, tendo em mente que $A_{\hat{H}}=\|H\|$ Id e $A_{\eta}=-$ Id. Das condições acima, portanto, segue-se que $U \subset x+V$, e $V=T_{y} N \oplus \operatorname{span}\{\widetilde{H}(y)\}=$ $W(y), \forall y \in U$.

Se agora $H(x)=0$ para todo $x \in N$, ou seja, se $N$ é totalmente geodésica, temos que $N^{k}$ está contida na interseç̧ão de $\mathbb{H}^{n}$ com um $(k+1)$ subespaço vetorial de $\mathbb{L}^{n+1}$, o qual nada mais é que $T_{x} N \oplus \operatorname{span}\{\eta(x)\}$. Logo, $N^{k} \subset x+W(x), \forall x \in N$.

Em resumo, temos que:

a) $x \longmapsto W(x)=T_{x} N \oplus \operatorname{span}\{\widetilde{H}(x)\}$ é localmente constante;

b) $\forall x \in N$ existe uma vizinhança $U(x)$ tal que $U(x) \subset x+W(x)$.

Sendo $N$ conexa, segue-se que $N^{k} \subset x+W(x)$, qualquer que seja $x \in N$.

As subvariedades umbílicas do espaço hiperbólico são essencialmente de três tipos, dependendo do sinal da curvatura seccional (que é constante, pela equação de Gauss; calcularemos essas curvaturas na seção seguinte): as esferas geodésicas (curvatura positiva), as equidistantes (curvatura negativa) e as horoesferas (planas). Essas subvariedades umbílicas também estão relacionadas com o caráter causal dos subespaços afins que as determinam, nos 
termos do teorema acima. Passaremos agora a descrevê-las, ressaltando essa relação com esses subespaços (na verdade, nos restringiremos ao caso das hipersuperfícies, pois o caso geral é completamente análogo, uma vez que as subvariedades umbílicas de formas espaciais reais têm codimensão essencial 1).

Esferas geodésicas. Dado $p \in \mathbb{H}^{n}$ e um número real $r>0$, define-se a esfera geodésica de centro $p$ e raio $r$

$$
\mathrm{S}^{n-1}(p, r):=\left\{(\cosh r) p+(\operatorname{senh} r) v \mid v \in T_{p} \mathbb{H}^{n},\langle v, v\rangle=1\right\} .
$$

Em outras palavras, $\mathrm{S}^{n-1}(p, r)$ é a imagem da $(n-1)$-esfera de raio $r$ de $T_{p} \mathbb{H}^{n}$ através da aplicação exponencial.

Note que $\mathbf{S}^{n-1}(p, r)$ está contida no subespaço afim $x+W$, onde $x$ é um ponto qualquer de $\mathbf{S}^{n-1}(p, r)$ e $W$ é o subespaço de $\mathbb{L}^{n+1}$ paralelo a $T_{p} \mathbb{H}^{n}$. De fato, tome uma base $\left\{v_{0}, v_{1}, \ldots, v_{n-1}\right\}$ de $W$. Dado $y \in \mathbf{S}^{n-1}(p, r)$, temos

$$
\begin{gathered}
y=(\cosh r) p+(\operatorname{senh} r)\left(\sum_{i=0}^{n-1} t_{i} v_{i}\right)= \\
=\left((\cosh r) p+(\operatorname{senh} r)\left(\sum_{i=0}^{n-1} s_{i} v_{i}\right)\right)+(\operatorname{senh} r)\left(\sum_{i=0}^{n-1}\left(t_{i}-s_{i}\right) v_{i}\right)
\end{gathered}
$$

que é da forma $x+v, x \in \mathbf{S}^{n-1}(p, r), v \in W$.

O subespaço vetorial $W \subset \mathbb{L}^{n+1}$ é claramente riemanniano, sendo paralelo a $T_{p} \mathbb{H}^{n}$. Vice-versa, dado um $(n-1)$-subespaço riemanniano $W \subset \mathbb{L}^{n+1}$, um subespaço afim paralelo a $W$ que "corta" $\mathbb{H}^{n}$ o faz segundo uma $(n-1)$-esfera geodésica, cujo centro é determinado pelo único subespaço afim paralelo a $W$ que tangencia $\mathbb{H}^{n}$ (essa afirmação, assim como as suas análogas nos dois casos seguintes, será melhor compreendida na próxima seção, ao falarmos de funções distância). Portanto, as esferas geodésicas são justamente as subvariedades umbílicas determinadas pelas intersecções de $\mathbb{H}^{n}$ com subespaços afins riemannianos de $\mathbb{L}^{n+1}$.

Hipersuperfícies equidistantes. Dado um hiperplano ${ }^{1} \mathcal{H}^{n-1} \subset \mathbb{H}^{n}$, seja $\xi \in \mathfrak{X}\left(\mathbb{H}^{n}\right)$ um campo unitário normal a $\mathcal{H}$ (se $W$ é o subespaço tal que

\footnotetext{
${ }^{1}$ No decorrer deste trabalho, sempre que falarmos em um hiperplano do espaço hiperbólico, estaremos nos referindo a uma hipersuperfície completa totalmente geodésica.
} 
$\mathcal{H}=W \cap \mathbb{H}^{n}$, então $\xi$ é a restrição a $\mathcal{H}$ do campo unitário (paralelo) normal a $W$ ). Para cada $r \in \mathbb{R}$, define-se a hipersuperfície equidistante

$$
\mathrm{H}^{n-1}(\mathcal{H}, r):=\{x=(\cosh r) z+(\operatorname{senh} r) \xi \mid z \in \mathcal{H}\} .
$$

Com um certo abuso de linguagem, $\mathrm{H}^{n-1}(\mathcal{H}, r)$ é uma hipersuperfície "paralela" a $\mathcal{H}$, e a distância entre as duas é constante e igual a $r$.

De modo análogo ao anterior, vê-se que $\mathbf{H}^{n-1}(\mathcal{H}, r)$ está contida no subespaço afim (ou linear) $x+W$, onde $x$ é um ponto qualquer de $\mathbf{H}^{n-1}(\mathcal{H}, r)$ (o artifício é exatamente o mesmo, escrevendo $\left.t_{i} v_{i}=s_{i} v_{i}+\left(t_{i}-s_{i}\right) v_{i}\right)$.

O subespaço $W$ é lorentziano, pois intercepta $\mathbb{H}^{n}$. Vice-versa, um subespaço afim paralelo a um dado $n$-subespaço lorentziano $W$ determina, em $\mathbb{H}^{n}$, hipersuperfícies equidistantes "paralelas" ao hiperplano $W \cap \mathbb{H}^{n}$. Portanto, as hipersuperfícies equidistantes são justamente as subvariedades umbílicas determinadas pelas interseç̧ões de $\mathbb{H}^{n}$ com subespaços afins (ou lineares) lorentzianos de $\mathbb{L}^{n+1}$.

Horoesferas. Dado $p \in \mathbb{H}^{n}$, seja $\gamma: \mathbb{R} \rightarrow \mathbb{H}^{n}$ uma geodésica qualquer, parametrizada por comprimento de arco, com $\gamma(0)=p$. Para cada $t>0$, considere a esfera geodésica de centro $\gamma(t)$ e raio $t$ (isto é, passando por $p)$. Como vimos anteriormente, essas esferas são do tipo $(p+W(t)) \cap \mathbb{H}^{n}$, onde $W(t)$ são subespaços riemannianos ortogonais a $\gamma(t)$. Note que, para $t$ tendendo a infinito, $\operatorname{span}\{\gamma(t)\}$ tende a uma direção $\operatorname{span}\{q\}$ paralela ao cone de luz. Define-se, então, a horoesfera

$$
\mathbf{R}^{n-1}(q, p):=\lim _{t \rightarrow \infty} \mathbf{S}^{n-1}(\gamma(t), t) .
$$

Os subespaços $W(t)$ acima convergem, para $t$ tendendo a infinito, ao subespaço $W$ ortogonal a $q$. Logo, $W$ é degenerado. Vice-versa, subespaços afins paralelos a um $n$-subespaço degenerado determinam horoesferas "paralelas" (num sentido que será mais claro mais adiante) do $\mathbb{H}^{n}$. Portanto, as horoesferas são justamente as subvariedades umbílicas determinadas pelas intersecções de $\mathbb{H}^{n}$ com subespaços afins degenerados de $\mathbb{L}^{n+1}$. 


\subsection{Funções Distância}

Nesta seção veremos alguns resultados relacionados a funções distância que serão particularmente úteis no decorrer do trabalho. A seção está baseada nos trabalhos de Thomas Cecil e Patrick Ryan em [Ce] e [CeRy].

Dado $q \in \mathbb{L}^{n+1} \backslash\{0\}$ e $x \in \mathbb{H}^{n} \backslash\{q\}$, está bem determinada a geodésica $\gamma_{x, q}=\operatorname{span}\{x, q\} \cap \mathbb{H}^{n}$. Define-se, então, o feixe de geodésicas determinado por $q$ como sendo o conjunto

$$
\mathcal{F}_{q}:=\left\{\gamma_{x, q} \mid x \in \mathbb{H}^{n} \backslash\{q\}\right\} .
$$

Note que $\gamma_{x, t q}=\gamma_{x, q}, \forall t \in \mathbb{R}$. Portanto, podemos sempre supor (e o faremos) $q \in \mathbb{H}^{n}, \mathbb{S}_{1}^{n}$ ou $\mathbb{V}_{+}^{n}$, onde

$$
\mathbb{V}_{+}^{n}=\left\{x \in \mathbb{V}^{n} \mid x_{n+1}>0\right\}
$$

$\mathrm{e}$

$$
\mathbb{S}_{1}^{n}=\left\{x \in \mathbb{L}^{n+1} \mid\langle\langle x, x\rangle\rangle=1\right\} .
$$

Dependendo do caráter causal de $q$, temos três tipos de feixes:

1. se $q \in \mathbb{H}^{n}, \mathcal{F}_{q}$ é simplesmente o feixe de geodésicas que passam por $q$, isto é,

$$
\mathcal{F}_{q}=\left\{\gamma(t)=(\cosh t) q+(\operatorname{senh} t) \xi \mid \xi \in T_{q} \mathbb{H}^{n}\right\}
$$

2. se $q \in \mathbb{S}_{1}^{n}, \mathcal{F}_{q}$ é o feixe de geodésicas ortogonais ao hiperplano $\mathcal{H}_{q}:=$ $\left\{x \in \mathbb{H}^{n} \mid\langle\langle x, q\rangle\rangle=0\right\}$, chamado de feixe de ultraparalelas e descrito por

$$
\mathcal{F}_{q}=\left\{\gamma(t)=(\cosh t) x+(\operatorname{senh} t) q \mid x \in \mathcal{H}_{q}\right\}
$$

3. se $q \in \mathbb{V}_{+}^{n}, \mathcal{F}_{q}$ é dito feixe de paralelas; as geodésicas deste nem se interceptam (como também acontece no feixe de ultraparalelas) nem têm um hiperplano ortogonal comum (para uma descrição de $\mathcal{F}_{q}$ nesse caso, veja a Observação 2.9 abaixo).

Nos itens (2) e (3) acima, as afirmações quanto à existência ou não de um hiperplano ortogonal às geodésicas do feixe se justificam como segue:

(2) Como $x$ é ortogonal a $T_{x} \mathbb{H}^{n}$ e $\gamma \subset \operatorname{span}\{x, q\}$, de $\langle\langle x, q\rangle\rangle=0 \quad \forall x \in \mathcal{H}_{q}$ segue-se que $\gamma^{\prime}=q$ em $x$ e, portanto, que $\gamma$ é ortogonal a $\mathcal{H}_{q}$; 
(3) Se existisse um hiperplano $\mathcal{H}_{p}$ ortogonal às geodésicas de $\mathcal{F}_{q}$, teríamos $\mathcal{F}_{p}=\mathcal{F}_{q}$, o que implicaria $p$ e $q$ linearmente dependentes. Mas $p$ é tipo espaço e $q$ é vetor nulo.

Já as descrições dos feixes, tanto nos casos (1) e (2), como no terceiro caso (veja Observação 2.9), decorrem de contas simples, tendo em mente as propriedades dos feixes e o fato de que a geodésica $\gamma_{x, q}$ se escreve como combinação linear de $x$ e $q$.

Lema 2.7 Dado $q \in \mathbb{V}^{n}$, o conjunto $h_{q}=\left\{x \in \mathbb{H}^{n} \mid\langle\langle x, q\rangle\rangle=-1\right\}$ é uma horoesfera. Vice-versa, dada uma horoesfera $h$, existe $q \in \mathbb{V}^{n}$ tal que $h=h_{q}$, e esse $q$ é único em $\mathbb{V}_{+}^{n}$.

\section{Demonstração:}

O subespaço afim $\left\{x \in \mathbb{L}^{n+1} \mid\langle\langle x, q\rangle\rangle=-1\right\}$ é paralelo ao subespaço degenerado $\left\{x \in \mathbb{L}^{n+1} \mid\langle\langle x, q\rangle\rangle=0\right\}$. Portanto, pelo que vimos anteriormente, $h_{q}$ é uma horoesfera.

Vice-versa, seja agora $h=(x+W) \cap \mathbb{H}^{n}$, com $x \in h$ e $W$ degenerado. Sem perda de generalidade, suponhamos que $W=\operatorname{span}\left\{e_{1}+e_{n+1}, e_{2}, \ldots, e_{n}\right\}$, onde $\left\{e_{i}\right\}$ é a base canônica de $\mathbb{L}^{n+1}$. Então, se $x=\left(x_{1}, \ldots, x_{n+1}\right) \in h$, tome $q=(k, 0, \ldots, 0, k)$, onde $k=1 /\left(x_{n+1}-x_{1}\right)$ (note que $x_{n+1}-x_{1} \neq 0$, pois $\left.x \in \mathbb{H}^{n}\right)$. Resulta, $\forall u, t_{i} \in \mathbb{R}, i=2, \ldots, n$ :

$$
\begin{gathered}
\left\langle\left\langle x+u\left(e_{1}+e_{n+1}\right)+t_{2} e_{2}+\cdots+t_{n} e_{n}, q\right\rangle\right\rangle= \\
=\langle\langle x, q\rangle\rangle+u\left\langle\left\langle e_{1}, q\right\rangle\right\rangle+u\left\langle\left\langle e_{n+1}, q\right\rangle\right\rangle= \\
=k x_{1}-k x_{n+1}+u k-u k=-1 .
\end{gathered}
$$

Logo, $(x+W) \cap \mathbb{H}^{n}=h_{q}$. A unicidade é trivial.

Observação 2.8 Para visualizar melhor qual a horoesfera $h_{q}$, dado $q=$ $(k, 0, \ldots, 0, k) \in \mathbb{V}^{n}$, tome $q^{\prime}=(-1 / 2 k, 0, \ldots, 0,1 / 2 k) \in \mathbb{V}^{n}$ e tome $W$ o $n$-subespaço tangente ao cone em $q$. Então, $h_{q}=\left(q^{\prime}+W\right) \cap \mathbb{H}^{n}$.

Observação 2.9 Com o lema acima, não é difícil ver que o feixe de paralelas determinado por $q \in \mathbb{V}_{+}^{n}$ pode ser descrito por

$$
\mathcal{F}_{q}=\left\{(\cosh t) x+(\operatorname{senh} t)(q-x) \mid x \in h_{q}\right\} .
$$


Seja agora $q \in \mathbb{H}^{n}, \mathbb{S}_{1}^{n}$ ou $\mathbb{V}_{+}^{n}$. Em cada um dos casos, define-se a função distância $L_{q}: \mathbb{H}^{n} \rightarrow \mathbb{R}$ dada por

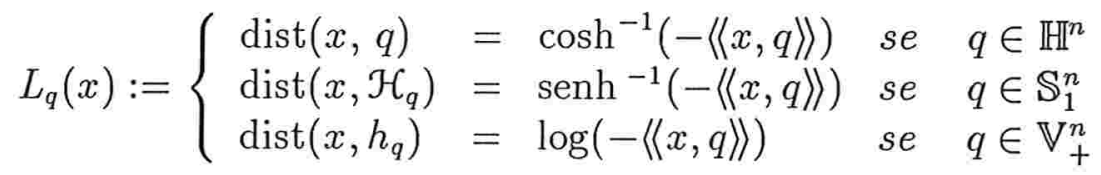

onde estamos tomando a função $\cosh ^{-1}$ a valores em $\mathbb{R}^{+}$.

Observação 2.10 Note que $L_{q}$ é não-negativa se $q \in \mathbb{H}^{n}$, mas assume qualquer valor nos outros dois casos. Trata-se, nesses casos, de uma espécie de "distância orientada" $\left(L_{q}^{-1}(r)\right.$ e $L_{q}^{-1}(-r)$ estão ambos a distância $r$ de $L_{q}^{-1}(0)$, mas em direções opostas).

A proposição seguinte é parte do Teorema do Índice para funções distância (cf. [CeRy], Teorema C, pg. 71) e caracteriza (parcialmente, mas quanto basta aos nossos objetivos) os pontos críticos das funções $L_{q}$.

Proposição 2.11 Seja $N^{k}$ uma subvariedade mergulhada de $\mathbb{H}^{n}$ e seja $q \in$ $\mathbb{H}^{n}, \mathbb{S}_{1}^{n}$ ou $\mathbb{V}_{+}^{n}$. Então, $x \in N$ é ponto crítico de $L_{q_{\left.\right|_{N}}}$ se e somente se existe um campo normal unitário $\xi \in T_{x} N^{\perp}$ e $t \in \mathbb{R}$ tal que

$$
q=\left\{\begin{array}{lll}
(\cosh t) x+(\operatorname{senh} t) \xi & \text { se } & q \in \mathbb{H}^{n} \\
(\operatorname{senh} t) x+(\cosh t) \xi & \text { se } & q \in \mathbb{S}_{1}^{n} \\
e^{t}(x+\xi) & \text { se } & q \in \mathbb{V}_{+}^{n}
\end{array}\right.
$$

\section{Demonstração:}

Caso 1: $q \in \mathbb{H}^{n}$.

Seja $x \in N^{k} \backslash\{q\}$ ( $q$ é ponto crítico de $L_{\left.q\right|_{N}}$ se e somente se $N=\{q\}$ ), e seja $U \subset N^{k} \backslash\{q\}$ uma vizinhança de $x$. Para cada $y \in U$, existe um único $\xi(y) \in T_{y} \mathbb{H}^{n}$ unitário tal que

$$
q=(\cosh r(y)) y+(\operatorname{senh} r(y)) \xi(y),
$$

onde $r(y)=L_{q}(y)=\cosh ^{-1}(-\langle\langle y, q\rangle\rangle)$. De fato, basta tomar o vetor unitário tangente, em $y$, à única geodésica de $\mathbb{H}^{n}$ que une $y$ a $q$. Agora, dado qualquer $X \in \mathfrak{X}(U)$, tem-se

$$
\left(\mathrm{d} L_{q}\right)_{y}(X)=X\left(L_{q}(y)\right)=X\left(\cosh ^{-1}(-\langle\langle y, q\rangle\rangle)\right)=
$$

\footnotetext{
${ }^{2}$ Dado o caráter local dessa proposição, essa hipótese não é restritiva.
} 


$$
=\frac{-X\langle\langle y, q\rangle\rangle}{\sqrt{\langle\langle y, q\rangle\rangle^{2}-1}}=-\frac{\langle\langle X, q\rangle\rangle}{\sqrt{\langle\langle y, q\rangle\rangle^{2}-1}} .
$$

Por outro lado,

$$
\begin{gathered}
\langle\langle X, q\rangle\rangle(y)=(\cosh r(y))\langle\langle X(y), y\rangle\rangle+(\operatorname{senh} r(y))\langle\langle X(y), \xi(y)\rangle\rangle= \\
=(\operatorname{senh} r(y))\langle\langle X(y), \xi(y)\rangle\rangle .
\end{gathered}
$$

Portanto, sendo $r(y) \neq 0, \forall y \in U$,

$$
\left(\mathrm{d} L_{q}\right)_{x}(X)=0 \Leftrightarrow\langle\langle X(x), q\rangle\rangle=0 \Leftrightarrow\langle\langle X(x), \xi\rangle\rangle=0
$$

o que prova, sendo $X$ genérico, o primeiro caso. Os casos seguintes são análogos, e serão mostrados sinteticamente.

Caso 2: $q \in \mathbb{S}_{1}^{n}$.

Na notação anterior, defina

$$
\xi(y):=\frac{1}{\cosh r(y)}(q-(\operatorname{senh} r(y)) y),
$$

onde agora $r(y)=\operatorname{senh}^{-1}(-\langle\langle y, q\rangle\rangle)$. O campo $\xi$ é o único campo unitário tangente a $\mathbb{H}^{n}$ ao longo de $U$ tal que

$$
q=(\operatorname{senh} r(y)) y+(\cosh r(y)) \xi(y), \quad \forall y \in U .
$$

O restante da prova é obtido calculando $\left(\mathrm{d} L_{q}\right)_{y}(X)$ como no caso anterior, levando em conta a expressão de $q$ acima.

Caso 3: $q \in \mathbb{V}_{+}^{n}$.

Nesse caso, basta tomar

$$
\xi(y)=-y+e^{-r(y)} q,
$$

$\operatorname{com} r(y)=\log (-\langle\langle y, q\rangle\rangle)$, e proceder como nos casos anteriores. 


\subsubsection{Umbílicas como conjuntos de nível}

Com esse instrumental à disposição, podemos dar uma interessante caracterização das hipersuperfícies umbílicas do espaço hiperbólico. Trata-se de vê-las como cunjuntos de nível das funções distância acima definidas. Essa e outras propriedades são o conteúdo das proposições seguintes.

Proposição 2.12 Dado $q \in \mathbb{H}^{n}$, seja $\mathbf{S}^{n-1}(q, r)=\left\{x \in \mathbb{H}^{n} \mid L_{q}(x)=r\right\}$. Então, para $r, s>0$,

1. $\mathrm{S}^{n-1}(q, r)$ é uma hipersuperfície umbílica compacta de curvatura seccional constante $1 / \operatorname{senh}^{2}(r)$, i.e., uma esfera geodésica;

2. o feixe de geodésicas passando por $q$ é ortogonal a $\mathrm{S}^{n-1}(q, r)$;

3. a distância entre $\mathbf{S}^{n-1}(q, r)$ e $\mathbf{S}^{n-1}(q, s)$ é $|r-s|$;

4. toda hipersuperfície umbílica completa de curvatura seccional positiva é da forma $\mathbf{S}^{n-1}(q, r)$, com q e r unicamente determinados.

Proposição 2.13 Dado $q \in \mathbb{S}_{1}^{n}$, seja $\mathbf{H}^{n-1}(q, r)=\left\{x \in \mathbb{H}^{n} \mid L_{q}(x)=r\right\}$. Então, para $r, s \in \mathbb{R}$,

1. $\mathrm{H}^{n-1}(q, r)$ é uma hipersuperfície umbílica completa de curvatura seccional constante $-1 / \cosh ^{2}(r)$, i.e., uma hipersuperfície equidistante;

2. o feixe de geodésicas ultraparalelas $\mathcal{F}_{q}$ é ortogonal a $\mathbf{H}^{n-1}(q, r)$;

3. a distância entre $\mathbf{H}^{n-1}(q, r)$ e $\mathbf{H}^{n-1}(q, s) e ́|r-s|$;

4. toda hipersuperfície umbílica completa de curvatura seccional negativa é da forma $\mathbf{H}^{n-1}(q, r)$, com o par $(q, r)$ unicamente determinado, a menos do sinal.

Proposição 2.14 Dado $q \in \mathbb{V}_{+}^{n}$, seja $\mathbf{R}^{n-1}(q, r)=\left\{x \in \mathbb{H}^{n} \mid L_{q}(x)=r\right\}$. Então, para $r, s \in \mathbb{R}$,

1. $\mathbf{R}^{n-1}(q, r)$ é uma hipersuperfície umbílica completa de curvatura seccional constante zero, i.e., uma horoesfera;

2. o feixe de geodésicas paralelas $\mathcal{F}_{q}$ é ortogonal a $\mathbf{R}^{n-1}(q, r)$;

3. a distância entre $\mathbf{R}^{n-1}(q, r)$ e $\mathbf{R}^{n-1}(q, s)$ é $|r-s|$; 
4. toda horoesfera é da forma $\mathbf{R}^{n-1}(q, r)$, com $(q, r)$ unicamente determinado, a menos de fatores escalares de $q$.

\section{Demonstração:}

Demonstraremos as três proposições simultaneamente.

Pela expressão de $L_{q}$ em qualquer um dos casos, tem-se que $L_{q}$ é constante se e somente se $\langle\langle\cdot, q\rangle\rangle$ é constante. Logo, os conjuntos de nível das funções $L_{q}$ são da forma

$$
L_{q}^{-1}(r)=\left\{x \in \mathbb{L}^{n+1} \mid\langle\langle x, q\rangle\rangle=k(r)\right\} \cap \mathbb{H}^{n},
$$

ou seja, são intersecções de $\mathbb{H}^{n}$ com subespaços afins $n$-dimensionais de $\mathbb{L}^{n+1}$. Isso mostra que os conjuntos de nível são esferas geodésicas, hipersuperfícies equidistantes ou horoesferas, dependendo de $q$ ser tipo tempo, tipo espaço ou tipo luz, respectivamente.

Quanto às curvaturas seccionais, usaremos a Proposição 2.11: se $N^{n-1}$ indica um dos conjuntos de nível $\mathbf{S}^{n-1}(q, r), \mathbf{H}^{n-1}(q, r)$ ou $\mathbf{R}^{n-1}(q, r)$, então $\forall x \in$ $N$, existe $\xi \in T_{x} N^{\perp}$ tal que

$$
q=\left\{\begin{array}{lll}
(\cosh r) x+(\operatorname{senh} r) \xi & \text { se } q \in \mathbb{H}^{n} \\
(\operatorname{senh} r) x+(\cosh r) \xi & \text { se } q \in \mathbb{S}_{1}^{n} \\
e^{r}(x+\xi) & \text { se } q \in \mathbb{V}_{+}^{n}
\end{array}\right.
$$

A curvatura seccional é dada por $K=-1+\lambda^{2}$ (cf. Equação de Gauss 1.10), onde $\lambda$ é o autovalor do operador de Weingarten da inclusão $N^{n-1} \hookrightarrow \mathbb{H}^{n}$. Logo, a menos de sinal, temos $\lambda=\left\langle\nabla_{X} \xi, X\right\rangle$, onde $X \in \mathfrak{X}(N)$ é um campo tangente qualquer. Da afirmação acima, resulta

$$
\nabla_{X} \xi=\left\{\begin{array}{lll}
-\operatorname{coth} r X & \text { se } & q \in \mathbb{H}^{n} \\
-\tanh r X & \text { se } & q \in \mathbb{S}_{1}^{n} \\
-X & \text { se } & q \in \mathbb{V}_{+}^{n}
\end{array}\right.
$$

Donde os valores de curvatura enunciados, provando o item (1) das proposições.

Ainda na notação acima, temos que $q, x$ e $\xi$ são coplanares (olhando em $\mathbb{L}^{n+1}$ ). Logo, a geodésica de $\mathbb{H}^{n}$ que passa por $x$ com "velocidade" $\xi$ está no 
plano gerado por $q$ e $x$, ou seja, é a própria $\gamma_{x, q}$. Como $\xi$ é normal a $N$ em $x$, fica provado o item (2) das proposições.

O item (3) é trivial, e o item (4) segue de considerações já feitas (note, para o caso da Proposição 2.14, que $\mathbf{R}^{n-1}(q, r)=\mathbf{R}^{n-1}(\lambda q, r+\log \lambda)$.

Observação 2.15 Com relação à notação usada, note que a hipersuperfície equidistante $\mathbf{H}^{n-1}\left(\mathcal{H}_{q}, r\right)$ corresponde à hipersuperfície de nível $\mathbf{H}^{n-1}(q, r)$, enquanto que a horoesfera $\mathbf{R}^{n-1}(q, p)$ corresponde a $\mathbf{R}^{n-1}(q, r)$, onde $r=$ $\log (-\langle\langle q, p\rangle\rangle)$. Já no caso da esfera geodésica a notação é a mesma.

\subsubsection{Imersões contidas em hipersuperfícies umbílicas}

Concluindo essa seção, veremos um lema que será usado diretamente na demonstração do teorema principal. O lema dá condições suficientes para que uma subvariedade imersa em $\mathbb{H}^{n}$ esteja contida numa hipersuperfície umbílica (este lema é a única exceção ao resto da seção, no sentido que não encontra-se nas referências citadas; a demonstração foi feita ad hoc, mesmo se é razoável acreditar que existem outras por aí afora).

Lema 2.16 Dada $f: N^{k \geq 2} \rightarrow \mathbb{H}^{n}$ uma imersão isométrica, com $N$ conexa, se existe uma direção normal $\eta$ umbílica e $\nabla^{\perp}$-paralela, então $f\left(N^{k}\right)$ está contida numa hipersuperfície umbílica de $\mathbb{H}^{n}$. Além do mais, $\eta$ é também a direção normal da imersão dessa hipersuperfície em $\mathbb{H}^{n}$.

Demonstração:

Seja $\lambda$ tal que $A_{\eta}=\lambda I$. Podemos supor $\lambda \geq 0$, trocando $\eta$ por $-\eta$ se necessário. Pela equação de Codazzi 1.8, sendo $k \geq 2$ e $\nabla_{X}^{\frac{1}{X}} \eta=0 \forall X \in$ $\mathfrak{X}(N)$, tem-se que $\lambda$ é constante.

Indicando com $D$ e $\nabla$, respectivamente, as conexões de Levi-Civita de $\mathbb{L}^{n+1}$ e $\mathbb{H}^{n}$, temos, pela fórmula de Gauss 2.1:

$$
D_{X} \eta=\nabla_{X} \eta=-A_{\eta} X
$$


Fixado $p \in N$, seja $U \subset N$ uma vizinhança de $p$ tal que $f_{\left.\right|_{U}}$ seja um mergulho. a) Se $\lambda>1$, seja $t \in \mathbb{R}$ tal que $\lambda=\operatorname{coth} t$ e seja $h_{+}: U \rightarrow \mathbb{H}^{n}$ dada por

$$
h_{+}(x)=(\cosh t) x+(\operatorname{senh} t) \eta .
$$

Assim

$$
\begin{gathered}
\mathrm{d} h_{+}(X)=(\cosh t) X-(\operatorname{senh} t) A_{\eta} X=(\cosh t-\lambda \operatorname{senh} t) X=0 \\
\therefore \quad h_{\left.\right|_{U}} \equiv \text { constante }=q \in \mathbb{H}^{n} .
\end{gathered}
$$

b) Se $0 \leq \lambda<1$, seja $t \in \mathbb{R}$ tal que $\lambda=\tanh t$ e seja $h_{-}: U \rightarrow \mathbb{S}_{1}^{n}$ dada por

$$
h_{-}(x)=(\operatorname{senh} t) x+(\cosh t) \eta .
$$

Temos

$$
\begin{gathered}
\mathrm{d} h_{-}(X)=(\operatorname{senh} t) X-(\cosh t) A_{\eta} X=(\operatorname{senh} t-\lambda \cosh t) X=0 \\
\therefore \quad h_{-\left.\right|_{U}} \equiv \text { constante }=q \in \mathbb{S}_{1}^{n} .
\end{gathered}
$$

c) Se $\lambda=1$ seja $h_{0}: U \rightarrow \mathbb{V}_{+}^{n}$ dada por

$$
h_{0}(x)=x+\eta
$$

Resulta

$$
\begin{gathered}
\mathrm{d} h_{0}(X)=X-A_{\eta} X=(1-\lambda) X=0 \\
\therefore \quad h_{\left.0\right|_{U}} \equiv \text { constante }=q \in \mathbb{V}_{+}^{n} .
\end{gathered}
$$

Da Proposição 2.11 segue-se, em qualquer um dos casos, que $\left(\mathrm{d} L_{q}\right)_{x} \equiv 0 \forall x \in$ $U$. Logo,

$$
0=\mathrm{d} L_{q}(X)=\left\langle\operatorname{grad} L_{q}, X\right\rangle \quad \forall X \in \mathfrak{X}(U) .
$$

Portanto, o aberto $U$ está contido em uma hipersuperfície de nível $\Omega^{n-1}$ de $L_{q}$, isto é, numa esfera geodésica (caso $\lambda>1$ ), numa hipersuperfície equidistante (caso $0 \leq \lambda<1$ ) ou numa horoesfera (caso $\lambda=1$ ). Enfim, como $N$ é conexa, resulta $N^{k} \subset \Omega^{n-1}$. 
Quanto à última afirmação do enunciado, observe que, em qualquer caso, $q$ é combinação linear de $x$ e $\eta$, o que mostra que $\eta$ é tangente ao plano gerado por $x$ e $q$. Como é também tangente a $\mathbb{H}^{n}$, segue-se que $\eta$ é tangente à geodésica $\gamma_{x, q}$ do feixe determinado por $q$. Pelo item (2) das Proposições 2.12, 2.13 e 2.14 , o campo $\eta$ é normal a $\Omega^{n-1}$.

\subsection{Hipersuperfícies de Revolução}

A definição de hipersuperfície de revolução que adotaremos neste trabalho é aquela de [dCDa]. Nesta seção, apresentaremos algumas propriedades básicas das hipersuperfícies de revolução. Terminaremos a seção com uma proposição que terá um papel importante na demonstração do teorema principal.

Dado um subespaço vetorial $W \subset \mathbb{L}^{n+2}$, indicaremos com $S O_{W}^{+}$o subgrupo de $\mathrm{SO}^{+}(1, n+1)$ constituido pelas isometrias que deixam fixo pontualmente $W$. No que segue, seja dada uma variedade riemanniana $M^{n}$ completa e conexa, e seja $f: M^{n} \rightarrow \mathbb{H}^{n+1}$ uma imersão isométrica.

Definição 2.17 A variedade $M$ (ou a imersão $f$ ) é chamada de hipersuperfície de revolução se existe um subespaço bidimensional $W \subset \mathbb{L}^{n+2}$ tal que $f(M)$ seja invariante por $S O_{W}^{+}$.

Observação 2.18 Em [dCDa], os autores definem as hipersuperfícies de revolução geradas por uma curva, numa ótica, digamos, construtiva ([dCDa], def. 2.2, pg. 687, ou [Suz], def. 3.2.1, pg. 58). A definição acima (que difere só formalmente da outra) é mais própria ao nosso caso, onde a variedade $M$ é um dos dados do problema, não devendo ser construída.

Pela Propriedade 2.4, os subespaços afins paralelos a $W^{\perp}$ são invariantes pela ação de $S O_{W}^{+}$e, portanto, contêm os paralelos (isto é, as $S O_{W}^{+}$-órbitas) de $f(M)$. Como $S O_{W}^{+}$age transitivamente sobre as intersecções $\left(x+W^{\perp}\right) \cap \mathbb{H}^{n+1}$, 
estas representam exatamente esses paralelos. Isso significa que os paralelos de $M$ são $(n-1)$-subvariedades umbílicas de $\mathbb{H}^{n+1}$, e o tipo de umbílica será determinado pelo caráter causal do plano $W$. Desse modo, dizemos que $f: M^{n} \rightarrow \mathbb{H}^{n+1}$ é uma hipersuperfície de revolução do tipo esférico, hiperbólico ou parabólico se $W$ for, respectivamente, lorentziano, riemanniano ou degenerado.

É também interessante observar que, em cada um dos casos, o grupo $S O_{W}^{+}$é naturalmente isomorfo a $S O(n)$ (caso esférico), $S O^{+}(1, n-1)$ (caso hiperbólico) ou $S O(n-1) \ltimes \mathbb{R}^{n-1}$. Não iremos provar isso aqui (veja [Suz] para maiores detalhes), mesmo porque trata-se somente de informação adicional, mas note que é razoável esperar que $S O_{W}^{+}$seja isomorfo, respectivamente, ao grupo (conexo) das isometrias da esfera $\mathbb{S}^{n-1}$, do espaço hiperbólico $\mathbb{H}^{n-1}$ ou do espaço euclidiano $\mathbb{R}^{n-1}$.

Resumindo, a fim de facilitar futuras consultas, podemos sintetizar as considerações feitas até aqui na seguinte tabela:

\begin{tabular}{|c|c|c|c|}
\hline$W$ & paralelos & tipo rotacional & $S O_{W}^{+}$ \\
\hline \hline \multirow{2}{*}{ lorentziano } & esf. geodésicas & esférico & $S O(n)$ \\
\hline \multirow{2}{*}{ riemanniano } & equidistantes & hiperbólico & $S O^{+}(1, n-1)$ \\
\hline \multirow{2}{*}{ degenerado } & horoesferas & parabólico & $S O(n-1) \ltimes \mathbb{R}^{n-1}$ \\
\hline
\end{tabular}

As hipersuperfícies de revolução têm uma estrutura local de produto torcido (excluindo eventuais pontos fixos). De fato, por um lado, uma hipersuperfície de revolução é uma variedade de co-homogeneidade 1 (decorre da própria definição). Por outro lado, pela Proposição 1.31, ela é localmente um produto do tipo $I \times \Sigma$, onde $I \subset \mathbb{R}$ e $\Sigma$ é uma órbita principal. A direção tangente a $I$ é geodésica e as órbitas principais são homotéticas entre si. A 
métrica de produto torcido é obtida levando em consideração a razão homotética das órbitas principais.

Um caso particular é o próprio espaço hiperbólico $\mathbb{H}^{n}$, o qual pode ser visto como hipersuperfície de revolução de $\mathbb{H}^{n+1}$. Para ver isso, seja $p \in \mathbb{L}^{n+2}$ tal que $\mathbb{H}^{n}=\mathcal{H}_{p}$ e considere o subespaço $(\operatorname{span}\{p\})^{\perp}$, o qual identificaremos com $\mathbb{L}^{n+1}$. Tome $q \in \mathbb{L}^{n+1}$ em um dos três conjuntos $\mathbb{H}^{n}, \mathbb{S}_{1}^{n}$ ou $\mathbb{V}_{+}^{n}$ (conforme a seção anterior). Enfim, tomando $W=\operatorname{span}\{p, q\} \subset \mathbb{L}^{n+2}$, obtemos $\mathbb{H}^{n}$ como uma hipersuperfície de revolução (do tipo esférico, hiperbólico ou parabólico, dependendo da escolha de $q$ ). Na verdade, porém, o espaço hiperbólico pode ser expresso globalmente como produto torcido, e isso veremos na próxima seção.

Finalizamos essa seção com a seguinte

Proposição 2.19 Seja $\Sigma^{n-1}$ uma $(n-1)$-subvariedade umbílica de $\mathbb{H}^{n+1}$, e seja $\bar{W}$ o n-plano afim de $\mathbb{L}^{n+2}$ tal que $\Sigma^{n-1}=\bar{W} \cap \mathbb{H}^{n+1}$. Seja $\widetilde{G}$ um subgrupo de Lie conexo de Iso $\left(\mathbb{H}^{n+1}\right)$. Se $\widetilde{G}$ age transitivamente sobre $\Sigma^{n-1}$, então:

(i) $\bar{W}$ é $\widetilde{G}$-invariante (i.e. $\widetilde{G}(\bar{W})=\bar{W}$ );

(ii) se $\Sigma$ não é totalmente geodésica, então Fix $\widetilde{G}=W^{\perp}$ (e, portanto, $\widetilde{G} \subset$ $\left.S O_{W^{\perp}}^{+}\right)$, onde $W^{\perp}$ é o 2-plano pela origem ortogonal a $\bar{W}$; se $\Sigma$ é totalmente geodésica, vale a inclusão Fix $\widetilde{G} \subset W^{\perp}$.

\section{Demonstração:}

Seja $W \subset \mathbb{L}^{n+2}$ o subespaço vetorial paralelo a $\bar{W}$ e seja $p \in \mathbb{L}^{n+2}$ tal que $\bar{W}=p+W$. Podemos supor $p \in \Sigma$. Dado $g \in \widetilde{G}$, temos

$$
g(\Sigma)=\Sigma=\bar{W} \cap \mathbb{H}^{n+1} .
$$

Por outro lado, temos

$$
g(\Sigma)=g\left(\bar{W} \cap \mathbb{H}^{n+1}\right)=g(\bar{W}) \cap \mathbb{H}^{n+1} .
$$

Portanto, $g(\bar{W})=\bar{W}$, o que prova (i).

Quanto à parte (ii), provemos primeiro que Fix $\widetilde{G} \subset W^{\perp}$. Para isso, note 
que, posto $V:=\operatorname{span}\{v \in W \mid p+v \in \Sigma\}$, então $V=W$. De fato, se assim não fosse, teríamos $\operatorname{dim} V \leq n-1$, daí

$$
n-1=\operatorname{dim} \Sigma=\operatorname{dim}\left((p+V) \cap \mathbb{H}^{n+1}\right) \leq n-2 .
$$

Agora, como $\widetilde{G}$ age transitivamente sobre $\Sigma$, temos que se $v \in W$ é tal que $p+v \in \Sigma$, então existe $g \in \widetilde{G}$ tal que $g(p+v)=p$. Então, $\forall x \in$ Fix $\widetilde{G}$, resulta

$$
\begin{gathered}
\langle\langle x, v\rangle\rangle=\langle\langle x,(p+v)-g(p+v)\rangle\rangle=\langle\langle x, p+v\rangle\rangle-\langle\langle x, g(p+v)\rangle\rangle= \\
=\langle\langle g(x), g(p+v)\rangle\rangle-\langle\langle g(x), g(p+v)\rangle\rangle=0 .
\end{gathered}
$$

Como $W=\operatorname{span}\{v \in W \mid p+v \in \Sigma\}$, segue-se que $x \in W^{\perp}$, isto é, Fix $\widetilde{G} \subset W^{\perp}$.

Para provar a outra inclusão, assumamos que $\Sigma$ não é totalmente geodésica (veja a Observação 2.20 a seguir sobre o caso em que $\Sigma$ é totalmente geodésica).

Se $W$ é não-degenerado, temos $W \cap W^{\perp}=\{0\}$ e $\bar{W} \cap W^{\perp}=\{q\}$, para um certo $q \in \mathbb{L}^{n+2}$. Como $\Sigma$ não é totalmente geodésica, temos $q \neq 0$. De (i), e da Propriedade 2.5, segue-se que $q \in$ Fix $\widetilde{G}$. Seja $q^{\prime} \perp q$ tal que $W^{\perp}=\operatorname{span}\left\{q, q^{\prime}\right\}$. Como $q \in \operatorname{Fix} \widetilde{G}$, segue-se que $g\left(q^{\prime}\right)= \pm q^{\prime} \forall g \in \widetilde{G}$. Pela conexidade de $\widetilde{G}$, devemos ter $q^{\prime} \in$ Fix $\widetilde{G}$ (de fato, podemos escrever $\widetilde{G}=$ $\left\{g \mid g\left(q^{\prime}\right)=q^{\prime}\right\} \cup\left\{g \mid g\left(q^{\prime}\right)=-q^{\prime}\right\} ;$ como $i d \in \widetilde{G}$, resulta $\left.\widetilde{G}=\left\{g \mid g\left(q^{\prime}\right)=q^{\prime}\right\}\right)$. Logo, Fix $\widetilde{G}=W^{\perp}$.

Se $W$ é degenerado, temos $W \cap W^{\perp}=\mathbb{R} v,\langle\langle v, v\rangle\rangle=0$ e $\bar{W} \cap W^{\perp}=\varnothing$ (pois $\left.p \notin W+W^{\perp}\right)$. Definamos

$$
W^{\prime}:=\operatorname{span}\{x \mid x \in \bar{W}\}=\mathbb{R} p \oplus W
$$

que é um $(n+1)$-subespaço lorentziano $\widetilde{G}$-invariante (pois $\widetilde{G}(\bar{W})=\bar{W}$ ). Temse $\left(W^{\prime}\right)^{\perp}=\mathbb{R} q$, para um certo $q$ unitário (tipo espaço). Como $q \in W^{\perp} \mathrm{e}$ $\widetilde{G}(\mathbb{R} q)=\mathbb{R} q$, segue-se que $q \in$ Fix $\widetilde{G}$ (pelos mesmos argumentos de conexidade de $\widetilde{G})$. Agora, como $W^{\perp}=\operatorname{span}\{q, v\}$ e $\widetilde{G}\left(W^{\perp}\right)=W^{\perp}$, resulta $\widetilde{G}(\mathbb{R} v)=$ $\mathbb{R} v$. Portanto, $\exists k: \widetilde{G} \rightarrow \mathbb{R}$ tal que $g(v)=k(g) v \quad \forall g \in \widetilde{G}$. Se mostrarmos 
que $v \in$ Fix $\widetilde{G}$ teremos completado a prova de (ii) e da proposição.

Dado $x \in \bar{W}, \exists y \in W$ tal que $x=p+y$. Logo,

$$
\langle\langle x, v\rangle\rangle=\langle\langle p, v\rangle\rangle \forall x \in \bar{W} .
$$

Em particular,

$$
g(x) \in \bar{W} \Rightarrow\langle\langle g(x), v\rangle\rangle=\langle\langle p, v\rangle\rangle
$$

Assim,

$$
\langle\langle p, v\rangle\rangle=\langle\langle x, v\rangle\rangle=\langle\langle g(x), g(v)\rangle\rangle=k(g)\langle\langle g(x), v\rangle\rangle=k(g)\langle\langle p, v\rangle\rangle .
$$

Mas $p \notin W^{\perp}$, ou seja $\langle\langle p, v\rangle \neq 0$. Logo, $k(g)=1 \forall g \in \widetilde{G}$ e $v \in \operatorname{Fix} \widetilde{G}$.

Observação 2.20 Na proposição acima, se $\Sigma$ é totalmente geodésica podemos afirmar o seguinte. Como $\mathbb{L}^{n+2}=W \oplus W^{\perp}$, temos também $\widetilde{G}=$ $\widetilde{G}_{W} \oplus \widetilde{G}_{W \perp}$, com óbvio significado dos termos. Em outras palavras, a igualdade da proposição pode não se realizar devido ao fato de $\widetilde{G}$ poder agir sobre $W$ e $W^{\perp}$ de forma "independente".

\subsection{O Espaço Hiperbólico como Produto Tor- cido}

Em [Nö], Nölker classifica as decomposições das formas espaciais reais em produtos torcidos. Em particular, para $\mathbb{H}^{n+1}$, ele obtém as seguintes decomposições (Exemplo 9, item d (iii), pgs. 11 e 12):

1. $\mathbb{H}_{*}^{n+1}=(0, \infty) \times_{\text {senh }} \mathbb{S}^{n}$, onde as fibras são esferas geodésicas $\left(\mathbb{H}_{*}^{n+1}\right.$ está indicando o espaço hiperbólico menos um ponto qualquer);

2. $\mathbb{H}^{n+1}=\mathbb{R} \times_{\cosh } \mathbb{H}^{n}$, onde as fibras são hipersuperfícies equidistantes;

3. $\mathbb{H}^{n+1}=\mathbb{R} \times \exp \mathbb{R}^{n}$, onde as fibras são horoesferas. 
Note que nas decomposições acima está embutido o fato de que as cópias da base $\mathbb{R}\left(\mathbb{R}^{+}\right.$no caso 1 ) correspondem a geodésicas (semigeodésicas no caso 1) ortogonais às fibras, ou seja, estas correspondem às hipersuperfícies de nível de uma oportuna função distância. Num certo sentido, é como olhar $\mathbb{H}^{n+1}$ na forma $L_{q}^{-1}(\mathbb{R})\left(\right.$ ou $\left.L_{q}^{-1}\left(\mathbb{R}^{+}\right)\right)$, para um certo $q \in \mathbb{L}^{n+2}$.

Observação 2.21 Embora o trabalho de Nölker seja mais do que suficiente para os nossos propósitos, ele requer uma leitura atenta, não imediata. Por esse motivo, vale dizer que há outro modo de se chegar às expressões acima, talvez não muito elegante, mas sem dúvida mais simples. Trata-se de olhar $\mathbb{H}^{n+1}$ como uma variedade de co-homogeneidade 1 (veja as considerações feitas na Seção 2.4, logo antes da Proposição 2.19) e, conhecendo as curvaturas seccionais das órbitas $(\lambda(t)=-\operatorname{coth} t, \quad-\tanh t, \quad-1$, respectivamente, se as órbitas forem esferas geodésicas, equidistantes, ou horoesferas), usar a expressão abaixo para o cálculo dos fatores de torção em cada um dos casos (cf. Proposição 3.10):

$$
\phi(t)=\exp \left(-\int_{0}^{t} \lambda(u) \mathrm{d} u\right)
$$

Enfim, mesmo se trivial, não custa observar também que os produtos torcidos acima podem, na verdade, ter um fator de torção "reparametrizado" (reparametrizando a geodésica normal às fibras). Isto é:

- $I \times_{\operatorname{senh} t} S^{n}$ é isométrico a $(s+I) \times_{\operatorname{senh}(t-s)} S^{n}\left(\right.$ desde que $\left.s+I \subset \mathbb{R}^{+}\right)$;

- $\mathbb{R} \times \operatorname{cosht} \mathbb{H}^{n}$ é isométrico a $(s+\mathbb{R}) \times_{\cosh (t-s)} \mathbb{H}^{n}$;

- $\mathbb{R} \times_{\exp (t)} \mathbb{R}^{n}$ é isométrico a $(s+\mathbb{R}) \times_{\exp (t-s)} \mathbb{R}^{n}$. 
Capítulo 3

Ações de Co-homogeneidade 1 com Órbitas Principais

Umbílicas

e Hipersuperfícies de Revolução de $\mathbb{H}^{n+1}$ 
O problema aqui estudado, como dissemos na Introdução, se insere no problema (bem) mais geral do estudo das imersões isométricas de $G$-variedades riemannianas em formas espaciais.

Apresentaremos, na primeira seção, a classificação das hipersuperfícies homogêneas do espaço euclidiano e do espaço hiperbólico (sobre o caso esférico, nos limitaremos a tecer alguns comentários).

O nosso objetivo é o de estender, a hipersuperfícies do espaço hiperbólico, o seguinte

Teorema 3.1 ([Se], Teorema 4.2.4, pg.65) Seja $M^{n}$ uma variedade riemanniana completa de G-co-homogeneidade $1, n \geq 3, G \subset$ Iso $(M)$ compacto, e seja $f: M^{n} \rightarrow \mathbb{R}^{n+1}$ uma imersão isométrica não-plana no infinito. Se as órbitas principais são umbílicas em $M$, então $f$ é de revolução.

Neste teorema, dizer que $f$ é de revolução significa que $f(M)$ é invariante pela ação de $S O(n)$ em $\mathbb{R}^{n+1}$ (deixando fixa uma determinada reta), e também que as $G$-órbitas principais de $M$ imergem como $S O(n)$-órbitas principais de $\mathbb{R}^{n+1}$. Além do mais, há a seguinte

Definição 3.2 Uma imersão isométrica $f: M^{n} \rightarrow \mathbb{R}^{n+1}$ é não-plana no infinito se cada componente conexa de $\mathcal{P}=\left\{x \in M \mid R_{M}(X, Y)=0 \quad \forall X, Y \in\right.$ $\left.T_{x} M\right\}$ for relativamente compacta.

Vale lembrar que o teorema acima é, por sua vez, uma extensão do seguinte

Teorema 3.3 ([PoSp], Teorema 3.1, pg. 175) Seja $M^{n}$ uma variedade riemanniana compacta de $G$-co-homogeneidade $1, n \geq 4, G \subset$ Iso $(M)$ compacto, e seja $f: M^{n} \rightarrow \mathbb{R}^{n+1}$ uma imersão isométrica. Se as órbitas principais de $G$ são umbílicas em $M$, então $f$ é de revolução.

O resultado que obtivemos é bastante similar ao Teorema 3.1 de Seixas, mas aparecem alguns exemplos não-rotacionais (cabe dizer que, por motivos técnicos, consideramos somente variedades de dimensão $n \geq 4$ ). 


\subsection{Hipersuperfícies Homogêneas de Formas Espaciais Reais}

A classificação das hipersuperfícies homogêneas do espaço euclidiano começou com Kobayashi em [Kol], onde o autor considerava o caso compacto. Nagano e Takahashi, em [NaTa], estenderam o resultado de Kobayashi ao caso das hipersuperfícies completas, mas com uma restrição ao número tipo da imersão. Em $[R y]$, Ryan dá outra demonstração desse mesmo resultado. Enfim, a limitação ao número tipo é resolvida em [Ha] e [Ta1]. Neste último, Takahashi prova o seguinte resultado:

Teorema 3.4 Uma hipersuperfície homogênea conexa $f: M^{n \geq 3} \rightarrow \mathbb{R}^{n+1}$ é isométrica a $\mathbb{S}^{k} \times \mathbb{R}^{n-k}(0 \leq k \leq n)$. Se o número tipo de $f$ for maior do que 1, então $f$ é um mergulho.

No caso das hipersuperfícies homogêneas do espaço hiperbólico, Ryan demonstra, no mesmo trabalho já citado, um teorema de classificação análogo ao do caso euclidiano (com a mesma restrição ao número tipo). De forma independente, Takahashi dá uma classificação completa dessas hipersuperfícies em [Ta1] e [Ta2]. Ele obteve o seguinte resultado:

Teorema 3.5 Seja $f: M^{n \geq 3} \rightarrow \mathbb{H}^{n+1}(c)$ uma hipersuperfície homogênea conexa do espaço hiperbólico de curvatura c. Então, quanto ao número tipo $\tau$ de $f$, temos as seguintes possibilidades:

- $\tau(p)=n \quad \forall p \in M$;

- $\tau(p) \leq 1 \quad \forall p \in M$;

- $\tau(p)=2 \quad \forall p \in M \quad$ (somente se $n=3$ ).

$E$ quanto à variedade $M$ :

1. Se $\tau \leq 1$, então $M$ é isométrica a $\mathbb{H}^{n}(c)$;

2. Se $\tau \equiv n$ e f é umbílica, então $M$ é isométrica a $\mathbb{S}^{n}\left(c^{\prime}\right)$, com $c^{\prime}>0$, $\mathbb{R}^{n}$ ou $\mathbb{H}^{n}\left(c^{\prime}\right)$, com $0>c^{\prime}>c ;$

3. Se $\tau \equiv n$ e f não é umbílica, então $f(M)$ é isométrica a $\mathbb{S}^{k}\left(c_{1}\right) \times$ $\mathbb{H}^{n-k}\left(c_{2}\right)$, com $0<k<n$ e $1 / c_{1}+1 / c_{2}=1 / c$. Se $k \neq 1$, f é um mergulho; 
4. Se $\tau \equiv 2$ (caso $n=3$ ), então $M$ é uma B-variedade.

As B-variedades são variedades do tipo $\left(G, \mathrm{~d} s^{2}\right)$, onde $G$ é um grupo de matrizes do tipo

$$
\left(\begin{array}{ccc}
e^{t} & 0 & -\epsilon \\
0 & e^{-t} & \eta \\
0 & 0 & 1
\end{array}\right)
$$

e a métrica $\mathrm{d} s^{2}$ é dada por

$$
\mathrm{d} s^{2}=e^{-2 t} \mathrm{~d} \epsilon^{2}+e^{2 t} \mathrm{~d} \eta^{2}+\mathrm{d} t^{2} .
$$

Para maiores detalhes, veja [Ta2], Introdução e $\S 1$.

Por fim, uma menção ao caso esférico. Esse é um caso bem mais complicado do que os outros dois e, não sendo sequer essencial ao nosso trabalho, nos limitaremos a dar algumas referências. Partimos novamente de Ryan, em [Ry], onde ele classifica as hipersuperfícies homogêneas da esfera com restrições tanto ao número tipo quanto ao número de curvaturas principais distintas. Em seguida, em [HsLa] (talvez o trabalho mais importante nesse contexto), Hsiang e Lawson contribuem à classificação das hipersuperfícies (extrinsecamente) homogêneas e compactas via classificação dos grupos de Lie compactos que agem linearmente com co-homogeneidade 2. Uma boa referência é [TaTk].

\subsection{Discussão Preliminar}

Voltemos agora ao nosso problema, apresentado no início do capítulo, e vejamos qual a natureza específica dos dados e das hipóteses envolvidos.

A primeira questão que se coloca concerne o grupo que age sobre a variedade. Olhando os três tipos de rotacionais do espaço hiperbólico, vemos que, em dois deles, as órbitas não são compactas. Isso impõe, de imediato, que consideremos ações de grupos não-compactos sobre a nossa variedade.

A segunda questão concerne a hipótese de não-planaridade no infinito. Antes de mais nada, cabe falar da necessidade de tal hipótese. Tanto no 
caso euclidiano como no caso hiperbólico pode acontecer a seguinte situação. Imagine uma hipersuperfície de revolução que, fora de um compacto, se estenda em modo totalmente geodésico. É claro que uma tal hipersuperfície é amplamente deformável. Para evitar situações desse tipo, torna-se necessário algum controle sobre a parte plana (extrinsecamente) da hipersuperfície, e é essa a função da hipótese em questão.

Além de estender a Definição 3.2 a hipersuperfícies do espaço hiperbólico (na verdade, estenderemos a qualquer ambiente a curvatura constante), há que se considerar o fato de que, ao se lidar com grupos não compactos, a tentativa de controlar o comportamento da imersão através de alguma hipótese de compacidade só faz sentido se essa hipótese é feita para o espaço de órbitas, e não para toda a variedade. A seguir, a nova definição:

Definição 3.6 Dada uma $G$-variedade $M^{n}$ completa e conexa, uma variedade riemanniana $\bar{M}_{c}^{n+1}$ de curvatura seccional constante $c$ e uma imersão isométrica $f: M^{n} \rightarrow \bar{M}_{c}^{n+1}$, seja $\mathcal{P}$ o conjunto

$$
\mathcal{P}:=\left\{x \in M \mid R(X, Y)=\bar{R}(X, Y) \quad \forall X, Y \in T_{x} M\right\},
$$

onde $R$ e $\bar{R}$ são os tensores de curvatura de $M$ e $\bar{M}_{c}$, respectivamente. Indicando com $\pi$ a projeção canônica de $M$ sobre o espaço de órbitas $M / G$, dizemos que o par $(f, G)$ tem planaridade (extrínseca) transversalmente compacta $(P T C)$ se $\pi(\mathcal{P}) \neq M / G$ e se cada componente conexa de $\pi(\mathcal{P})$ for compacta.

Quando não houver dúvidas, omitiremos a menção ao grupo $G$ e, se for o caso, omitiremos também a menção à imersão $f$ e nos referiremos simplesmente à variedade $M$.

Observação 3.7 Note que, pela Equação de Gauss 1.7, $x \in \mathcal{P}$ se e somente se o número tipo $\tau(x)$ é menor ou igual a 1 . Note também que, pela Proposição 1.29 , se isso acontece em um ponto $x$, acontece em toda a órbita de $x$, isto é, $\mathcal{P}$ é $G$-invariante.

Quanto ao nome dado ao conceito acima, e ao motivo de não ter preservado o original de [Se] (na verdade, o original já tinha sido mudado), a opção de deixar de lado a menção ao "infinito", assim como a escolha do termo "transversal", vem do fato, já discutido acima, do controle da planaridade 
ser feito sobre o espaço de órbitas, e não mais sobre toda a variedade.

A essa altura, já poderíamos enunciar um teorema com as mesmas condições do Teorema 3.1, adaptadas ao nosso caso, esperando obter o mesmo resultado. Mas o caso hiperbólico oferece algumas surpresas: há exemplos de variedades de co-homogeneidade 1 com órbitas principais umbílicas que podem ser imersas isometricamente no espaço hiperbólico com PTC e que não são de revolução. Veremos na próxima seção esses exemplos, e em seguida daremos o enunciado do teorema para o caso hiperbólico.

\subsection{Exemplos Não Rotacionais}

Seja $\Omega^{n}$ uma hipersuperfície umbílica de $\mathbb{H}^{n+1}$ com curvatura seccional não-positiva, isto é, uma horoesfera ou uma hipersuperfície equidistante. Tendo em mente a classificação das hipersuperfícies homogêneas de $\mathbb{R}^{n} \mathrm{e}$ $\mathbb{H}^{n}$ (Seção 3.1, Teoremas 3.4 e 3.5, respectivamente), tome $\Sigma^{n-1} \hookrightarrow \Omega^{n}$ homogênea, não umbílica e com número tipo $\tau \geq 2$.

Defina $\Lambda$ como sendo a subvariedade de $\mathbb{H}^{n+1}$ constituída pelos pontos do feixe de geodésicas ortogonais a $\Omega$ ao longo de $\Sigma$, isto é

$$
\Lambda=\left\{\exp _{x} t X \mid x \in \Sigma, t \in \mathbb{R}\right\},
$$

onde $X \in \mathfrak{X}(\Omega)^{\perp}$ e $\|X\|=1$. Note que, como $\mathbb{H}^{n+1}$ pode se decompor como $\mathbb{R} \times_{\phi} \Omega$ para uma oportuna torção $\phi$ (cf. Seção 2.5), resulta $\Lambda=\mathbb{R} \times_{\phi} \Sigma$. Uma qualquer geodésica do feixe ortogonal a $\Omega$ será chamada de geratriz de $\Lambda$.

Afirmação: $\Lambda$ é uma variedade $n$-dimensional completa e conexa de cohomogeneidade 1 com órbitas principais umbílicas e a inclusão $\Lambda \hookrightarrow \mathbb{H}^{n+1}$ é uma imersão com PTC que não é de revolução.

É claro que $\Lambda$ é uma $n$-variedade conexa, e, pelo Lema 1.9, também completa. Vejamos agora que $\Lambda$ é de co-homogeneidade 1 com órbitas principais umbílicas.

Se $G$ indica o grupo de isometrias de $\Sigma$, então podemos estender a ação 
de $G$ a $\Lambda=\mathbb{R} \times_{\phi} \Sigma$ de modo natural: para cada $g \in G$ defina

$$
g(t, x):=(t, g(x))
$$

As órbitas (todas principais) dessa ação são as fibras $\Sigma_{t}=\{t\} \times \Sigma$, as quais têm codimensão 1 em $\Lambda$ e são umbílicas. Para ver que essa ação é de fato isométrica, basta provar que, para cada $t \in \mathbb{R}$, é isométrica a ação $G \times \Sigma_{t} \rightarrow$ $\Sigma_{t}$. A fibra $\Sigma_{t}$ é homotética a $\Sigma$ (a homotetia é a restrição a $\Sigma$ da homotetia entre $\Omega$ e $\{t\} \times \Omega)$ e essa homotetia é equivariante. Portanto, se $h$ indica a homotetia entre $\Sigma$ e $\Sigma_{t}$, a ação acima nada mais é que a composição $h \circ g \circ h^{-1}$. Assim, se $k$ é a razão homotética de $h$, para cada par de campos $Y, Z \in \mathfrak{X}\left(\Sigma_{t}\right)$ resulta.

$$
\begin{gathered}
\left\langle\mathrm{d}\left(h \circ g \circ h^{-1}\right)(Y), \mathrm{d}\left(h \circ g \circ h^{-1}\right)(Z)\right\rangle=k^{2}\left\langle\mathrm{~d}\left(g \circ h^{-1}\right)(Y), \mathrm{d}\left(g \circ h^{-1}\right)(Z)\right\rangle= \\
=k^{2}\left\langle\mathrm{~d} h^{-1}(Y), \mathrm{d} h^{-1}(Z)\right\rangle=\langle Y, Z\rangle .
\end{gathered}
$$

Para ver que $\Lambda$ não é uma hipersuperfície de revolução, basta observar que $\Sigma$ (assim como cada $\Sigma_{t}$ ) não é umbílica em $\mathbb{H}^{n+1}$, uma vez que não é umbílica em $\Omega$ (respectivamente, em $\{t\} \times \Omega$ ).

Para provar a afirmação resta a PTC. Esse também é um fato simples, mas vejamos com mais detalhes.

Seja $\eta$ o campo normal unitário ao longo da imersão $\Sigma \hookrightarrow \Omega$ e $A_{\eta}^{\prime}$ o relativo operador de forma. Por construção, $X$ é o campo normal a $\Sigma$ em $\Lambda$ e $\eta$ é o normal a $\Lambda$ em $\mathbb{H}^{n+1}$. Denote ainda com $A_{\eta}$ o operador de forma dessa última imersão. Esquematicamente:

$$
\Sigma^{n-1} \stackrel{X}{\longrightarrow} \Lambda^{n} \stackrel{\eta}{\longrightarrow} \mathbb{H}_{\eta} \mathbb{H}^{n+1}
$$

e

$$
\Sigma^{n-1} \stackrel{\eta}{\underset{A_{\eta}^{\prime}}{\longrightarrow}} \Omega^{n} \stackrel{X}{\longrightarrow} \mathbb{H}^{n+1}
$$


Mostraremos que $A_{\eta}$ e $A_{\eta}^{\prime}$ têm o mesmo posto, o que implica, em particular, a PTC (pela Observação 3.7, pois o posto de $A_{\eta}^{\prime}$ é maior ou igual a 2).

Indicando com $\tilde{\nabla}$ a conexão de Levi-Civita de $\mathbb{H}^{n+1}$, e sendo $\Omega \hookrightarrow \mathbb{H}^{n+1}$ umbílica, existe $\lambda \in \mathbb{R}$ tal que $\widetilde{\nabla}_{Y} X=-\lambda Y \quad \forall Y \in \mathfrak{X}(\Omega)$. Em particular, se $Y \in \mathfrak{X}(\Sigma)$, então

$$
A_{\eta} Y=A_{\eta}^{\prime} Y-\left\langle\widetilde{\nabla}_{Y} \eta, X\right\rangle X
$$

e

$$
\left\langle\widetilde{\nabla}_{Y} \eta, X\right\rangle=-\left\langle\eta, \widetilde{\nabla}_{Y} X\right\rangle=\lambda\langle\eta, Y\rangle=0 .
$$

Logo, $A_{\eta} Y=A_{\eta}^{\prime} Y \quad \forall Y \in \mathfrak{X}(\Sigma)$. Em particular, $A_{\eta} Y \in T \Sigma$ e, assim,

$$
\left\langle A_{\eta} X, Y\right\rangle=\left\langle X, A_{\eta} Y\right\rangle=0
$$

ou seja $A_{\eta} X=\mu X$, para um certo $\mu \in \mathbb{R}$. Agora,

$$
\mu=\left\langle A_{\eta} X, X\right\rangle=-\left\langle\widetilde{\nabla}_{X} \eta, X\right\rangle=\left\langle\eta, \widetilde{\nabla}_{X} X\right\rangle=0
$$

pois $X$ é o campo tangente a uma geodésica de $\mathbb{H}^{n+1}$. Assim, $A_{\eta} X=0$, e portanto o posto de $A_{\eta}$ é dado pela sua restrição a $T \Sigma$ que, como vimos, é o posto de $A_{\eta}^{\prime}$.

Observação 3.8 A mesma construção acima, que foi feita a partir de uma $\Omega$ com curvatura não-positiva, pode ser feita partindo de uma esfera geodésica. Nesse caso, obtém-se uma hipersuperfície com as mesmas características das acima, porém não completa. Em particular, num estudo de tipo local, esse exemplo também deve ser considerado.

\subsection{Teorema Principal}

Os exemplos desenvolvidos na seção anterior serão chamados, de agora em diante, de exemplos $N R$ (não é uma definição, é simplesmente para simplificar a escrita e a leitura do texto). Temos, enfim, todos os elementos necessários para enunciar o teorema principal desse trabalho. 
Teorema 3.9 Seja $M^{n \geq 4}$ uma variedade riemanniana completa e conexa, $G \subset$ Iso $(M)$ um subgrupo fechado e conexo que age com co-homogeneidade 1 sobre $M$ e seja $f: M^{n} \rightarrow \mathbb{H}^{n+1}$ uma imersão isométrica com planaridade extrínseca transversalmente compacta. Se as órbitas principais são umbílicas em $M$, então $f$ é de revolução (com as órbitas principais sendo mandadas em paralelos) ou $f(M)$ é congruente a um dos exemplos $N R$.

Acreditamos ser conveniente apresentar um esquema geral da demonstração, antes de iniciá-la efetivamente, para facilitar a tarefa de quem se propõe a lê-la. Aproveitaremos para antecipar alguns resultados que serão usados.

Uma característica importante que permeia praticamente toda a demonstração é dada pela seguinte proposição, provada inicialmente em [PoSp] mas que recebeu nova prova, mais direta, em [Se] (Proposição 4.1.8, pg. 54, enunciada para $G$ compacto, mas válida também para $G$ fechado):

Proposição 3.10 Seja $M^{n}$ uma G-variedade de co-homogeneidade 1 completa e conexa. Se as órbitas principais são umbílicas, então $M_{\text {reg }}$ tem estrutura local de produto torcido. Mais especificamente, dado $p \in M_{\text {reg }}$, seja $\sigma: \mathbb{R} \rightarrow M$ uma geodésica normal com $\sigma(0)=p$ e seja $S=\sigma(-\epsilon, \epsilon)$, para um certo $\epsilon$ positivo, uma fatia em $p$ (cf. Proposição 1.27). Então o tubo $G(S)$ é isométrico ao produto torcido $(-\epsilon, \epsilon) \times_{\phi} G(p)$, com $\phi:(-\epsilon, \epsilon) \rightarrow \mathbb{R}$ dada por

$$
\phi(t)=\exp \left(-\int_{0}^{t} \lambda(u) \mathrm{d} u\right)
$$

onde $\lambda(u)$ é o autovalor do operador de Weingarten da inclusão de $G(\sigma(u))$ em $M$.

A proposição acima é importante porque nos diz, em primeiro lugar, que as órbitas principais são homotéticas entre si e, em segundo, porque nos dá "boas" propriedades de curvatura (aquelas da Proposição 1.10 ou, pensando no nosso caso, da Observação 1.12).

Dito isso, vejamos agora como procederemos adiante. O primeiro passo é considerar o seguinte conjunto:

$$
\mathcal{R}:=\left\{x \in M_{\text {reg }} \mid \exists \Gamma \in \mathcal{U}_{G}(x) \text { tal que } f_{\left.\right|_{\Gamma}} \text { é de revolução }\right\},
$$

onde $\mathcal{U}_{G}(x)$ indica o conjunto das vizinhanças $G$-invariantes de $x$. 
Se $\mathcal{R} \neq \varnothing$, provaremos que é denso em $M_{\text {reg }}$ e que as órbitas principais imergem como umbílicas em hiperplanos de $\mathbb{H}^{n+1}$. Isso será feito em duas etapas, uma delas considerando o subconjunto $\{x \in \mathcal{R} \mid \tau(x) \geq 2\}$ e a outra o seu complementar $\{x \in \mathcal{R} \mid \tau(x) \leq 1\}$, onde $\tau$ indica o número tipo da imersão $f$.

Na primeira etapa, provaremos que as órbitas principais são umbílicas em $\mathbb{H}^{n+1}$ (usando o Lema 2.16) e usaremos o seguinte teorema, conseqüência da Proposição 2.19 (no decorrer dessa etapa se provará que $\tau \neq 2$ ):

Teorema 3.11 Seja $M^{n}$ uma $G$-variedade de co-homogeneidade 1, com $G \subset$ Iso $(M)$ fechado e conexo, com $M=M_{\text {reg }}$, e seja $f: M^{n} \rightarrow \mathbb{H}^{n+1}$ uma imersão isométrica com número tipo $\tau(x) \geq 3 \forall x \in M$. Se as órbitas (principais) de $M$ são umbílicas em $\mathbb{H}^{n+1}$, então $f$ é de revolução e as órbitas de $M$ são mandadas pela $f$ nos paralelos de $f(M)$.

No teorema acima, não estamos supondo $M$ completa. Antes de prová-lo, precisamos do seguinte lema:

Lema 3.12 Nas condições acima, $f$ induz um homomorfismo diferenciável $\phi: \operatorname{Iso}(M) \rightarrow \operatorname{Iso}\left(\mathbb{H}^{n+1}\right)$, dado pela relação

$$
f \circ g=\phi(g) \circ f .
$$

\section{Demonstração:}

Sendo $\tau \geq 3$, a imersão $f$ é rígida (cf. Teorema 1.5). Logo, $\forall g \in$ Iso $(M)$, existe $T_{g} \in$ Iso $\left(\mathbb{H}^{n+1}\right)$ tal que $f \circ g=T_{g} \circ f$. Agora, para ver que a $\phi$ está bem definida (isto é, que $T_{g}$ é única), seja dada uma isometria $g \in \operatorname{Iso}(M)$ e sejam $T_{1}, T_{2} \in$ Iso $\left(\mathbb{H}^{n+1}\right)$ tais que $T_{1} \circ f=T_{2} \circ f=f \circ g$. Então, tomando $T=T_{1}^{-1} \circ T_{2}$, temos $f(M) \subset \operatorname{Fix}(T)$. Da Propriedade 1.21, item (4), seguese que $f(M)$ está contida numa subvariedade totalmente geodésica de $\mathbb{H}^{n+1}$. Se fosse $\operatorname{dim}(\operatorname{Fix}(T))=n$, então $f$ seria totalmente geodésica em $\mathbb{H}^{n+1}$ e $\tau=0$. Logo, $\operatorname{Fix}(T)=\mathbb{H}^{n+1}$, o que implica $T_{1}=T_{2}$. Desse modo, $\phi$ está bem definida e é, trivialmente, um homomorfismo.

Resta mostrar que $\phi$ é diferenciável. Queremos agradecer a Daniel Tausk pelo argumento usado a seguir.

Antes de prosseguir, cabem algumas considerações gerais sobre $\mathbb{H}^{n+1}$. Sejam dados $n+2$ pontos $p_{1}, \ldots, p_{n+2} \in \mathbb{H}^{n+1}$ geometricamente independentes (isto 
é, linearmente independentes, se vistos como vetores de $\mathbb{L}^{n+2}$ ). Uma isometria de $\mathbb{H}^{n+1}$ fica univocamente determinada pelos valores que assume nesses pontos. De fato, a escolha de uma $(n+2)$-upla de pontos geometricamente independentes equivale à escolha de um ponto e de uma base do espaço tangente nesse ponto, e é sabido que duas isometrias do espaço hiperbólico que coincidem num ponto e que, ainda nesse ponto, têm a mesma diferencial são iguais.

Dito isso, a idéia é construir uma aplicação diferenciável $\rho:$ Iso $(M) \rightarrow$ $\left(\mathbb{H}^{n+1}\right)^{n+2}$ e um mergulho diferenciável $\psi:$ Iso $\left(\mathbb{H}^{n+1}\right) \rightarrow\left(\mathbb{H}^{n+1}\right)^{n+2}$ tais que $\psi \circ \phi=\rho$. Isso provaria a diferenciabilidade da $\phi$, concluindo a demonstração do lema.

Como $f$ não é totalmente geodésica (pois $\tau \geq 3$ ), $f(M)$ não pode estar contida em nenhum hiperplano de $\mathbb{H}^{n+1}$. Desse modo, existem $n+2$ pontos $p_{1}, \ldots, p_{n+2} \in \mathbb{H}^{n+1}$ geometricamente independentes com $p_{i}=f\left(x_{i}\right)$, para oportunos $x_{i} \in M, i=1, \ldots, n+2$. Definamos, então:

$$
\begin{aligned}
& \rho(g):=\left(\phi(g)\left(p_{1}\right), \ldots, \phi(g)\left(p_{n+2}\right)\right) \\
& \mathrm{e} \\
& \psi(T):=\left(T\left(p_{1}\right), \ldots, T\left(p_{n+2}\right)\right) .
\end{aligned}
$$

A $\psi$ é claramente diferenciável, e $\psi \circ \phi=\rho$. Quanto à diferenciabilidade da $\rho$, lembre que $\phi(g)\left(f\left(x_{i}\right)\right)=f\left(g\left(x_{i}\right)\right)$, para todo $i=1, \ldots, n+2$, e que, fixado $x \in M$, a aplicação $g \longmapsto g(x)$ de Iso $(M)$ em $M$ é diferenciável. Resta provar que $\psi$ é um mergulho.

Note, antes de mais nada, que $\psi$ é injetora (pelo que dissemos acima sobre pontos geometricamente independentes). Para provar que $\psi$ é um mergulho usaremos a seguinte idéia: construiremos uma aplicação diferenciável $\widetilde{\psi}:\left(\mathbb{H}^{n+1}\right)^{n+2} \rightarrow \operatorname{End}\left(\mathbb{L}^{n+2}\right)$ (onde End $\left(\mathbb{L}^{n+2}\right)$ indica o espaço dos endomorfismos de $\left.\mathbb{L}^{n+2}\right)$ tal que $\iota=\widetilde{\psi} \circ \psi$, onde $\iota$ é a inclusão de Iso $\left(\mathbb{H}^{n+1}\right)=$ $O^{+}(1, n+1)$ em End $\left(\mathbb{L}^{n+2}\right)$. Uma vez feito isso, teríamos:

1. $\mathrm{d}(\widetilde{\psi} \circ \psi)=\mathrm{d} \widetilde{\psi} \circ \mathrm{d} \psi=\mathrm{d} \iota$, implicando que $\mathrm{d} \psi$ é injetora, isto é, $\psi$ é uma imersão; 
2. $\psi^{-1}$ está bem definida e $\iota \circ \psi^{-1}=\widetilde{\psi}_{\left.\right|_{\left(\text {Iso } \mathbb{H}^{n+1}\right)}}$, implicando que $\psi^{-1}$ é contínua.

A aplicação em questão é bastante natural: dados $q_{1}, \ldots, q_{n+2} \in \mathbb{H}^{n+1}$, defini$\operatorname{mos} \widetilde{\psi}\left(q_{1}, \ldots, q_{n+2}\right)$ como sendo a aplicação linear de End $\left(\mathbb{L}^{n+2}\right)$ que manda $p_{i}$ em $q_{i}, i=1, \ldots, n+2$. Em termos de matrizes, essa aplicação é claramente diferenciável (as entradas são as componentes dos $q_{i}$ na base $\left\{p_{i}\right\}$ ) e ela satisfaz a relação desejada. Isso prova que $\psi$ é um mergulho diferenciável, o que, por sua vez, prova que $\phi$ é diferenciável, concluindo a demonstração do lema.

Observação 3.13 Note que, nas condições do Teorema 3.11, não podemos garantir que $\phi(G)$ seja fechado em Iso $\left(\mathbb{H}^{n+1}\right)$, isto é, que seja um subgrupo de Lie de Iso $\left(\mathbb{H}^{n+1}\right)$. Mesmo assim, $\phi(G)$ tem uma estrutura de grupo de Lie induzida pelo isomorfismo entre $\phi(G)$ e $G / \operatorname{ker} \phi_{\left.\right|_{G}}$ (este último é um grupo de Lie, pois $\operatorname{ker} \phi_{\left.\right|_{G}}$ é um subgrupo de Lie normal em $G$ ).

Passemos agora à demonstração do Teorema 3.11.

\section{Demonstração:}

Do lema acima, segue-se que as órbitas de $M$ são extrinsecamente homogêneas (isto é, são $\phi(G)$-homogêneas). Além do mais, nenhuma órbita é totalmente geodésica, pois, do contrário, teríamos pontos onde $\tau \leq 2$ (na verdade, para fins da demonstração, bastaria que uma órbita não fosse totalmente geodésica).

Por outro lado, para cada órbita $\Sigma$ existe um $n$-subespaço $W_{\Sigma} \subset \mathbb{L}^{n+2}$ tal que $\Sigma=\bar{W}_{\Sigma} \cap \mathbb{H}^{n+1}$, onde $\bar{W}_{\Sigma}$ é um subespaço afim paralelo a $W_{\Sigma}$.

Podemos então aplicar a Proposição 2.19 para cada órbita de $M$, do que se segue:

1. $\operatorname{dim}(\operatorname{Fix}(\phi(G)))=2$;

2. $\left(W_{\Sigma}\right)^{\perp}=\operatorname{Fix}(\phi(G)), \quad \forall \Sigma$.

Em outras palavras, os subespaços afins $\bar{W}_{\Sigma}$ são todos paralelos ao mesmo subespaço $W=(\operatorname{Fix}(\phi(G)))^{\perp}$. Logo, $f(M)$ é invariante por $S O_{\mathrm{Fix}(\phi(G))}^{+}$, e as órbitas são mandadas nos paralelos de $f(M)$. 
Feito isso, passaremos a estudar o subconjunto $\{x \in \mathcal{R} \mid \tau(x) \leq 1\}$. Essa parte é bastante delicada, e entraremos com mais detalhes quando chegar o momento.

A finalização dessa parte (lembrando, o caso $\mathcal{R} \neq \varnothing$ ) é feita através de uma oportuna "colagem" das informações já obtidas até aqui, mostrando que $f$ é de revolução.

Enfim, a última etapa concerne o caso $\mathcal{R}=\varnothing$. Este caso corresponde, como já se pode intuir, aos exemplos NR. A prova é bastante direta, usando alguns fatos que já terão sido demonstrados no estudo do primeiro caso (por sua vez baseados nas propriedades de curvatura dos produtos torcidos), e mostrando, ao menos implicitamente, as isometrias envolvidas.

Com essa descrição esquemática, esperamos ter contribuído a aliviar a leitura da demonstração propriamente dita, que é o assunto da próxima seção.

\subsection{Demonstração}

De agora em diante, seja $M^{n}$ uma variedade riemanniana completa e conexa, com $n \geq 4$, e $G \subset$ Iso $(M)$ um subgrupo fechado e conexo que age sobre $M$ com co-homogeneidade 1 e órbitas principais umbílicas. Indiquemos $\operatorname{com} \pi: M \rightarrow M / G$ a projeção canônica de $M$ sobre o espaço de órbitas $M / G$.

Seja $f: M^{n} \rightarrow \mathbb{H}^{n+1}$ uma imersão isométrica com PTC, e indiquemos com $\tau$ o número tipo da $f$. Dado $x \in M$, e indicando com $\Sigma_{x}=G(x)$ a órbita de $x$ (ou simplesmente $\Sigma$ quando não houver dúvidas), sejam dados os campos unitários $X \in T_{x} \Sigma^{\perp} \cap T_{x} M$ e $\xi \in T_{x} M^{\perp}$.

Indiquemos com $\nabla, \bar{\nabla}$ e $\widetilde{\nabla}$, respectivamente, as conexões de $\Sigma, M$ e $\mathbb{H}^{n+1}$. Quanto aos operadores de Weingarten das imersões envolvidas, sejam: $\bar{A}_{X}$ relativo à imersão $\Sigma \rightarrow M, A_{\xi}$ relativo à imersão $M \rightarrow \mathbb{H}^{n+1}$ e $\bar{A}_{X}, \bar{A}_{\xi}$ relativos à imersão $\Sigma \rightarrow \mathbb{H}^{n+1}$. Enfim, seja $\lambda$ tal que $\bar{A}_{X}=\lambda I$. 
Esquematicamente, temos:

$$
(\Sigma, \nabla) \underset{\bar{A}_{X}}{\stackrel{X}{\longrightarrow}}\left(M^{n}, \bar{\nabla}\right) \underset{A_{\xi}}{\stackrel{\xi}{\longrightarrow}}\left(\mathbb{H}^{n+1}, \widetilde{\nabla}\right)
$$

$\mathrm{e}$

$$
(\Sigma, \nabla) \underset{\bar{A}_{X}, \bar{A}_{\xi}}{\longrightarrow}\left(\mathbb{H}^{n+1}, \widetilde{\nabla}\right) .
$$

Como visto acima, seja

$$
\mathcal{R}:=\left\{x \in M_{\text {reg }} \mid \exists \Gamma \in \mathcal{U}_{G}(x) \text { tal que } f_{\left.\right|_{\Gamma}} \text { é de revolução }\right\},
$$

onde $\mathcal{U}_{G}(x)$ indica o conjunto das vizinhanças $G$-invariantes de $x$.

Enfim, consideremos a partição $M_{\text {reg }}=M_{\leq 1} \cup M_{>1}$, onde

$$
M_{\leq 1}:=\left\{x \in M_{\text {reg }} \mid \tau(x) \leq 1\right\}
$$

$\mathrm{e}$

$$
M_{>1}:=\left\{x \in M_{\text {reg }} \mid \tau(x) \geq 2\right\} .
$$

Note que, pela hipótese de PTN da imersão $f$, tem-se que $M_{>1} \neq \varnothing$.

\subsubsection{Caso $\mathcal{R} \neq \varnothing$}

Antes de iniciar, mais um pouco de nomenclatura. Usaremos o termo genérico $\delta$-umbílica, com $\delta$ assumindo os valores $-1,0$ ou 1, para indicar uma subvariedade umbílica de $\mathbb{H}^{n+1}$ com curvatura de mesmo sinal de $\delta$. E com uma expressão do tipo "as órbitas principais são (ou imergem como) $\delta$-umbílicas" estaremos entendendo que o $\delta$ é o mesmo para todas as órbitas principais compreendidas na expressão. Isso não é uma definição, mas apenas uma nomenclatura (bastante discutível, por sinal), cujo objetivo é o de simplificar o texto.

Enfim, um esclarecimento a respeito da notação. Usaremos ( ) $V$ para indicar a projeção sobre um dado espaço $V$. 
Etapa 1: $M_{>1} \subset \mathcal{R}$

Suponhamos $\mathcal{R} \neq \varnothing$ e provemos que $M_{>1} \subset \mathcal{R}$.

Lema 3.14 Se $\left(A_{\xi} X\right)_{T_{x} \Sigma} \neq 0$, então $\tau(x)=1$. Se $A_{\xi} X=\mu X$, com $\mu \neq 0$, então $\tau(x)=1$ ou $\tau(x)=n$.

\section{Demonstração:}

Seja $\Gamma=I \times_{\phi} \Sigma^{n-1}$ uma vizinhança $G$-invariante de $x$. Seja $Z \in T_{x} \Sigma$ unitário, tal que $A_{\xi} X=\mu X+k Z$, com $k \neq 0$. Pelas propriedades do produto torcido (Prop. 1.10, item (3)), temos $\forall Y \in T_{x} \Sigma$

$$
R_{M}(Y, Z) X=0 \text {. }
$$

E pela equação de Gauss da imersão $\Gamma \rightarrow \mathbb{H}^{n+1}$

$$
\begin{gathered}
R_{M}(Y, Z) X=-(Y \wedge Z) X+\left(A_{\xi} Y \wedge A_{\xi} Z\right) X= \\
=-\left\langle Y, A_{\xi} X\right\rangle A_{\xi} Z+\left\langle Z, A_{\xi} X\right\rangle A_{\xi} Y .
\end{gathered}
$$

Se $\langle Y, Z\rangle=0$, resulta $k A_{\xi} Y=0$. Logo,

$$
A_{\xi}\left(T_{x} \Gamma\right) \subset \operatorname{span}\left\{A_{\xi} X, A_{\xi} Z\right\} .
$$

Por outro lado, temos

$$
R_{M}(X, Y) X=-(X \wedge Y) X+\left(A_{\xi} X \wedge A_{\xi} Y\right) X=Y \quad \text { se }\langle Y, Z\rangle=0 .
$$

Ainda pela Prop. 1.10, item (2), temos

$$
R_{M}(X, Y) X=\left(\frac{1}{\phi} \operatorname{Hess}_{\phi}(X, X)\right) Y
$$

o que implica

$$
\frac{\operatorname{Hess}_{\phi}(X, X)}{\phi}=1 .
$$

Assim, $R_{M}(X, Z) X=Z$. Mas

$$
\begin{gathered}
R_{M}(X, Z) X=-(X \wedge Z) X+\left(A_{\xi} X \wedge A_{\xi} Z\right) X=Z-\mu A_{\xi} Z+k A_{\xi} X \\
\therefore \quad A_{\xi} X=\frac{\mu}{k} A_{\xi} Z \quad \text { ou seja } \quad \tau(x)=1 .
\end{gathered}
$$


Isso prova a primeira afirmação. Seja agora $A_{\xi} X=\mu X$. Resulta, então, $A_{\xi}\left(T_{x} \Sigma\right) \subset T_{x} \Sigma$, e $\forall Y \in T_{x} \Sigma$ temos dessa vez

$$
R_{M}(X, Y) X=\frac{\operatorname{Hess}_{\phi}(X, X)}{\phi} Y=Y-\mu A_{\xi} Y .
$$

Temos ainda,

$$
\mu \neq 0 \Rightarrow A_{\xi} Y=\frac{1}{\mu}\left(1-\frac{\operatorname{Hess}_{\phi}(X, X)}{\phi}\right) Y
$$

isto é

$$
\left(A_{\xi}\right)_{\left.\right|_{T_{x} \Sigma}}=k I \text {. }
$$

Logo, $r k\left(\left(A_{\xi}\right)_{\left.\right|_{T_{x} \Sigma}}\right)$ é 0 ou $n-1$. Ou seja

$$
r k\left(A_{\xi}\right)=1 \quad \text { ou } \quad r k\left(A_{\xi}\right)=n .
$$

Corolário 3.15 Se $\tau(x) \geq 2$, então $A_{\xi} X=\mu X$, e portanto $X$ e $\xi$ são $\nabla^{\perp}$-paralelos. Além do mais, se $\tau(x)=2, A_{\xi} X=0$.

\section{Demonstração:}

Imediata.

O Corolário 3.15, juntamente com o Lema 2.16, nos leva ao seguinte

Corolário 3.16 Se $\tau(x) \geq 2$, então $\exists \Omega^{n} \subset \mathbb{H}^{n+1}$ umbílica tal que $f(\Sigma) \subset \Omega$ e o operador de Weingarten de $\Sigma^{n-1} \rightarrow \Omega^{n}$ é dado por $\bar{A}_{\xi}$.

Demonstração:

$X$ é direção umbílica por hipótese $\left(\bar{A}_{X}=\lambda I\right)$ e, pelo Corolário 3.15, é também $\nabla^{\perp}$-paralela. O Lema 2.16 , portanto, garante que existe uma hipersuperfície umbílica $\Omega^{n} \subset \mathbb{H}^{n+1}$, com campo normal dado por $X$, tal que $f(\Sigma) \subset \Omega$. Sendo $X \in \mathfrak{X}(\Omega)^{\perp}$, o campo normal a $\Sigma$ em $\Omega$ deve ser $\xi$. E temos, para cada $Y \in \mathfrak{X}(\Sigma)$, indicando com $\nabla^{\prime}$ a conexão de $\Omega$,

$$
-\left(\nabla_{Y}^{\prime} \xi\right)_{T \Sigma}=-\left(\widetilde{\nabla}_{Y} \xi\right)_{T \Sigma}=\bar{A}_{\xi}(Y)
$$


provando a última afirmação.

Lema 3.17 Para todo $x \in M_{>1}, \Sigma_{x}$ é umbílica em $\mathbb{H}^{n+1}$.

Demonstração:

O fato de ser $\mathcal{R} \neq \varnothing$ implica que as órbitas principais têm curvatura seccional constante. De fato, existe um aberto de $M_{\text {reg }}$ que é de revolução, e portanto as órbitas desse aberto têm curvatura constante. Mas as órbitas principais de $M$ são homotéticas entre si, logo todas têm curvatura constante (cf. equação 1.11 , pg. 11). Sendo $\tau(x) \geq 2$, o Corolário 3.16 implica que $\Sigma_{x} \subset \Omega$, onde $\Omega$ é umbílica em $\mathbb{H}^{n+1}$ ( $\therefore \Omega$ também tem curvatura constante). Notando que $\bar{A}_{\xi}=\left(A_{\xi_{\mid T \Sigma}}\right)_{T \Sigma}$, temos, pelo Corolário 3.15

$$
r k\left(\left(A_{\left.\xi\right|_{T \Sigma}}\right)_{T \Sigma}\right)=\left\{\begin{array}{lll}
\tau-1 & \text { se } & \tau \neq 2 \\
\tau & \text { se } & \tau=2
\end{array}\right.
$$

ou seja, o número tipo da imersão $\Sigma \rightarrow \Omega$ é sempre maior ou igual a 2 . A conclusão segue então da Proposição 1.3.

Observação 3.18 Lembrando que as órbitas principais são positivamente homotéticas entre si (cf. seção dedicada aos produtos torcidos) e tendo em mente a equação 1.11 (pg. 11) que relaciona as curvaturas seccionais de variedades homotéticas, o lema acima mostra que as órbitas principais de $M_{>1}$ imergem como $\delta$-umbílicas em $\mathbb{H}^{n+1}$.

Enfim, temos o seguinte

Teorema 3.19 Para todo $x \in M_{>1}$, existe uma vizinhança $G$-invariante $\Gamma$ de $x$ tal que $f_{\mid \Gamma}$ é de revolução (e as órbitas são mandadas nos paralelos de $f(\Gamma)$ ), isto é, $M_{>1} \subset \mathcal{R}$.

\section{Demonstração:}

Pelo Lema 3.17, a imersão $\Sigma_{x} \rightarrow \mathbb{H}^{n+1}$ é umbílica. Logo, $\tau(x) \geq n-1$ (já que não podemos ter $\tau(x) \leq 1$ ). Como $n \geq 4$, temos $\tau(x) \geq 3$, ou seja, $\tau(x) \neq 2$. 
Seja $\Gamma \subset M_{\text {reg }}$ uma vizinhança $G$-invariante de $x$ tal que $\tau(y) \geq 3 \quad \forall y \in \Gamma$. $O$ resultado segue do Teorema 3.11 aplicado à imersão $\Gamma \rightarrow \mathbb{H}^{n+1}$.

Observação 3.20 Note que usamos a hipótese $\mathcal{R} \neq \varnothing$ pela primeira vez no Lema 3.17 acima, e somente para assegurar a existência de uma órbita principal com curvatura constante. Disso segue-se que:

1. $\mathcal{R} \neq \varnothing$ se e somente se as órbitas principais têm curvatura seccional constante. Em particular, é isso que acontece se existe $p \in M_{\text {reg }}$ tal que $\tau(p) \leq 1$ (pela equação de Gauss da imersão $\Sigma_{p} \rightarrow M, \Sigma_{p}$ tem curvatura $-1+\lambda^{2}$ ). Assim, temos também que, se $\mathcal{R}=\varnothing$, então $\tau(x) \geq 2 \quad \forall x \in M_{\text {reg }}$.

2. se $\mathcal{R}=\varnothing$, então valem os Corolários 3.15 e 3.16 .

Etapa $2: \stackrel{\circ}{M}_{\leq 1} \subset \mathcal{R}$

A demonstração que segue é inspirada em [PoSp] (cf. lemas 3.10, 3.11, $3.12,3.13$ e 3.14 , pgs. $179-183)$.

Aqui também precisamos de alguns preliminares. Seja $x \in M$ um ponto singular excepcional (se existir) e seja $N \in \mathcal{U}_{G}(x)$ uma qualquer vizinhança $G$-invariante de $x$. Defina

$$
\widetilde{N}=\left\{(y, v) \mid y \in N, v \in T_{y} \Sigma_{y}^{\perp} \mathrm{e}\langle v, v\rangle=1\right\}
$$

com a projeção $\kappa: \tilde{N} \rightarrow N$ dada por $\kappa((y, v))=y$. A ação de $G$ se estende naturalmente a $\tilde{N}$ da seguinte forma:

$$
g((y, v))=\left(g(y), g_{*}(v)\right), \quad \forall g \in G .
$$

Desse modo, $\widetilde{N}$ é uma $G$-variedade riemanniana de co-homogeneidade 1, e tem-se que $\operatorname{dim}(G(y, v))=\operatorname{dim}(G(y))$, para todo $y \in N$. Isto é, uma eventual $G$-órbita singular de $\widetilde{N}$ é excepcional. 
Agora tome $\sigma: I \rightarrow N$ um segmento de geodésica normal passando por $x$. Pelo Teorema 1.33, para todo $y \in \sigma(I)$ tem-se que $G_{y} \subset G_{x}$ e $G_{x} / G_{y}$ é difeomorfo a $\mathbb{S}^{0}\left(\because \operatorname{dim}\left(\Sigma_{y}\right)=\operatorname{dim}\left(\Sigma_{x}\right)\right)$, isto é $G_{y}$ tem índice 2 em $G_{x}$. Vejamos o que acontece com as isotropias $G_{(x, v)}$ e $G_{(y, v)}$ ( $v$ agora indica um campo unitário tangente a $\sigma(I))$.

Por um lado, sendo $y$ regular, para cada $g \in G$ está bem definido o campo $g_{*}(v)$, o qual é equivariante (por definição). Daí segue-se que

$$
g(y, v)=(y, v) \Leftrightarrow g(y)=y \text { e } g_{*}(v)=v \Leftrightarrow g(y)=y .
$$

Logo, $G_{(y, v)}=G_{y}$. Por outro lado, em $G_{x}$ podemos ter isometrias $g$ tais que $\mathrm{d} g_{x}=-\mathrm{Id}$. Logo, $G_{(x, v)}=\left\{g \in G_{x} \mid g_{*}=\mathrm{Id}\right\}$ tem índice $2 \mathrm{em} G_{x}$. Concluindo, $G_{(x, v)}=G_{(y, v)}$ e todas as órbitas de $\tilde{N}$ são regulares.

Passemos agora a outro ponto que será importante no decorrer da demonstração. Já sabemos que as órbitas principais são isométricas a $\delta$-umbílicas, para um certo $\delta$. O que iremos provar agora é a seguinte

Proposição 3.21 Se $\delta=0$ ou -1 , isto é, se as órbitas principais são isométricas a $\mathbb{R}^{n-1}$ ou $\mathbb{H}^{n-1}(c)$, então $M=M_{\text {reg }}$.

\section{Demonstração:}

Há um teorema de Cartan (Teorema 5.10 de [Ko2], pg. 66, ou Teorema 9.2 de [KoNo], pg. 111) que garante que toda ação de um grupo compacto sobre uma variedade riemanniana completa, simplesmente conexa de curvatura não-positiva tem ponto fixo.

Suponha, agora, por absurdo, que exista $p \in M$ ponto singular, e tome, numa fatia em $p$, um ponto regular $x$. Olhando a ação $G_{p} \times \Sigma_{x} \rightarrow \Sigma_{x}$, vê-se que não pode haver pontos fixos. De fato, se $y \in \operatorname{Fix}\left(G_{p}\right)$, então $G_{p} \subset G_{y}$ e, sendo $y$ regular, também $p$ o seria. Mas $G_{p}$ é compacto e $\Sigma_{x}$ satisfaz as condições do teorema de Cartan, logo devo ter $M=M_{\text {reg }}$.

Vejamos agora mais um pouco de notação. Seja $\gamma: \mathbb{R} \rightarrow M$ uma geodésica normal fixada e seja $J \subset \mathbb{R}$ um intervalo homeomorfo, via $\pi \circ \gamma$, a $\pi\left(M_{\text {reg }}\right)$. Pela Proposição 1.29, faz sentido a decomposição $J=J_{\leq 1} \cup J_{>1}$, onde

$$
J_{\leq 1}=\gamma^{-1}\left(M_{\leq 1}\right) \cap J=\{t \in J \mid \tau(\gamma(t)) \leq 1\}
$$


e

$$
J_{>1}=\gamma^{-1}\left(M_{>1}\right) \cap J=\{t \in J \mid \tau(\gamma(t)) \geq 3\} \neq \varnothing
$$

onde já usamos o fato de que $M_{>1} \neq \varnothing$ e $\tau(x) \neq 2 \quad \forall x \in M_{\text {reg }}$.

Iniciaremos agora com uma série de lemas que culminarão com a prova de que $\stackrel{\circ}{M}_{\leq 1} \subset \mathcal{R}$.

Antes do primeiro lema, vejamos um aspecto técnico que será útil no decorrer da demonstração. Defina, para cada $x \in \mathbb{H}^{n+1}$, o conjunto

$$
S_{x}:=\left\{y \in \mathbb{S}_{1}^{n+1} \mid\langle\langle y, x\rangle\rangle=0\right\}=(\operatorname{span}\{x\})^{\perp} \cap \mathbb{S}_{1}^{n+1} .
$$

$S_{x}$ é um subespaço topológico compacto de $\mathbb{S}_{1}^{n+1}$ e representa, módulo relação antipodal, o feixe de hiperplanos de $\mathbb{H}^{n+1}$ que passam por $x$. Fixado $x$, queremos determinar o $\max \left\{y_{n+2}^{2} \mid y=\left(y_{1}, \ldots, y_{n+2}\right) \in S_{x}\right\}$. É claro que esse máximo é invariante por transformações de Lorentz que deixam fixo o eixo da $(n+2)$-ésima coordenada. Portanto, podemos supor $x=\left(x_{1}, 0, \ldots, 0, x_{n+2}\right)$. Como $\langle\langle x, y\rangle\rangle=0$, segue-se que

$$
y_{n+2}=0 \quad \text { se } \quad x_{1}=0
$$

ou

$$
y_{1}=k y_{n+2}, \quad \text { onde } \quad k=x_{n+2} / x_{1} .
$$

No segundo caso, de $\langle\langle y, y\rangle\rangle=1$ temos

$$
k^{2} y_{n+2}^{2}+y_{2}^{2}+\cdots+y_{n+1}^{2}-y_{n+2}^{2}=1
$$

donde

$$
y_{n+2}^{2}=\frac{1}{k^{2}-1}\left(1-\sum_{i=2}^{n+1} y_{i}^{2}\right) .
$$

Logo, o valor máximo se obtém quando $y_{2}=\cdots=y_{n+1}=0$ e é dado por $y_{n+2}^{2}=x_{1}^{2}$. No primeiro caso, o máximo também é dado por $y_{n+2}^{2}=$ $x_{1}^{2}=0$. Enfim, voltando ao caso genérico $x=\left(x_{1}, x_{2}, \ldots, x_{n+2}\right)$, resulta $\max \left\{y_{n+2}^{2}\right\}=\sum_{i=1}^{n+1} x_{i}^{2}$. A conclusão que nos interessa aqui é que, se $K \subset \mathbb{H}^{n+1}$ é um compacto, então existe um compacto $B \subset \mathbb{S}_{1}^{n+1}$ tal que $S_{x} \subset B \quad \forall x \in K$. 
Lema 3.22 Se $t \notin \stackrel{\circ}{\leq 1}_{\leq 1}$, então $f\left(\Sigma_{\gamma(t)}\right)$ é uma $\delta$-umbílica contida num hiperplano de $\mathbb{H}^{n+1}$.

\section{Demonstração:}

Se $t \in J_{>1}$, então existe uma vizinhança $\Gamma \in \mathcal{U}_{\gamma(t)}$ tal que $f_{\left.\right|_{\Gamma}}$ é de revolução. Nesse caso, portanto, o lema é trivial.

Seja então $t \in J_{\leq 1} \backslash \stackrel{\circ}{J}_{\leq 1}\left(J_{\leq 1}=\overline{J_{\leq 1}}\right.$ é fechado), e seja $\left\{t_{k}\right\} \subset J_{>1}$ tal que $t_{k} \rightarrow t$. Para cada inteiro $k, f\left(\Sigma_{\gamma\left(t_{k}\right)}\right)$ é uma $\delta$-umbílica num hiperplano $\mathcal{H}_{k} \subset \mathbb{H}^{n+1}$. Seja então $q_{k} \in \mathbb{S}_{1}^{n+1}$ tal que $\mathcal{H}_{k}=\left\{x \in \mathbb{H}^{n+1} \mid\left\langle\left\langle x, q_{k}\right\rangle\right\rangle=0\right\}$ (o que indicamos anteriormente com $\left.\mathcal{H}_{q_{k}}\right)$. Se $K \subset \mathbb{H}^{n+1}$ é um compacto contendo $f\left(\gamma(t)\right.$ ) (e portanto contendo também $f\left(\gamma\left(t_{k}\right)\right)$ para todo $k$ maior que um certo $k_{0}$ ), então existe um compacto $B \subset \mathbb{S}_{1}^{n+1}$ tal que $S_{x} \subset B \quad \forall x \in K$. Mas $q_{k} \in S_{\gamma\left(t_{k}\right)}$, logo $\left\{q_{k}\right\} \subset B\left(\forall k>k_{0}\right)$. Podemos então supor que $q_{k}$ converge para um certo $q \in \mathbb{S}_{1}^{n+1}$.

Isso significa que os hiperplanos $\mathcal{H}_{k}$ convergem para o hiperplano $\mathcal{H}=\{x \in$ $\left.\mathbb{H}^{n+1} \mid\langle\langle x, q\rangle\rangle=0\right\}$, e conseqüentemente $f\left(\Sigma_{\gamma(t)}\right) \subset \mathcal{H}$. Como $\Sigma_{\gamma(t)}$ tem curvatura constante, então, se esta não for igual a -1 , a Proposição 1.3 implica que $f\left(\Sigma_{\gamma(t)}\right)$ é umbílica em $\mathcal{H}$.

Para o caso em que a curvatura de $\Sigma_{\gamma(t)}$ for -1 (o que só é possível no caso $\delta=-1)$, precisamos ver que a imersão $f: \Sigma_{\gamma(t)} \rightarrow \mathcal{H}$ é totalmente geodésica, (em princípio, poderia ter número tipo igual a 1). Temos que, para cada inteiro $k, f\left(\Sigma_{\gamma\left(t_{k}\right)}\right)$ é uma $(n-1)$-superfície equidistante (não totalmente geodésica, pois $\left.\tau\left(\gamma\left(t_{k}\right)\right) \geq 3\right)$ contida em $\mathcal{H}_{k}$. Note que $\mathcal{H}_{k}$ também se escreve como $L_{k}^{n+1} \cap \mathbb{H}^{n+1}$, onde $L_{k}^{n+1}=\left\{x \in \mathbb{L}^{n+2} \mid\left\langle\left\langle x, q_{k}\right\rangle\right\rangle=0\right\}=\left(\operatorname{span}\left\{q_{k}\right\}\right)^{\perp}$. Então, para cada inteiro $k$, existe $p_{k} \in L_{k}^{n+1} \cap S_{1}^{n+1}$ tal que, tomando $W_{k}=\left\{x \in L^{n+1} \mid\left\langle\left\langle x, p_{k}\right\rangle\right\rangle=0\right\}$, resulta $f\left(\Sigma_{\gamma\left(t_{k}\right)}\right)=\left(f\left(\gamma\left(t_{k}\right)\right)+W_{k}\right) \cap L_{k}^{n+1}=$ $\left(f\left(\gamma\left(t_{k}\right)\right)+W_{k}\right) \cap \mathbb{H}^{n+1}$. Observe que $W_{k}=\left(\operatorname{span}\left\{p_{k}, q_{k}\right\}\right)^{\perp}$ e que a seqüencia $\left\{\left(p_{k}, q_{k}\right)\right\}$ possui uma subseqüencia convergente (pelas mesmas razões usadas anteriormente). Logo, os subespaços $W_{k}$ convergem a um $n$-subespaço $W$ e tem-se que $f\left(\Sigma_{\gamma(t)}\right)=(f(\gamma(t))+W) \cap \mathbb{H}^{n+1}$ é uma $(n-1)$-subvariedade umbílica de $\mathbb{H}^{n+1}$ contida em $\mathcal{H}$, isto é, considerando que a curvatura de $\Sigma_{\gamma(t)}$ é $-1, f\left(\Sigma_{\gamma(t)}\right)$ é totalmente geodésica em $\mathcal{H}$.

Seja agora $t_{0} \in \stackrel{\circ}{J}_{\leq 1}$ fixado, e seja $I=(\alpha, \beta) \subset \stackrel{\circ}{J}_{\leq 1}$ o intervalo maximal contendo $t_{0}$. Temos que $G(\gamma(I))$ tem curvatura seccional constante $-1 \mathrm{e}$, sendo $M_{>1} \neq \varnothing$, não podemos ter $\gamma(\alpha)$ e $\gamma(\beta)$ ambos singulares. Sem perda 
de generalidade, suporemos $\gamma(\alpha)$ regular. Note que, pela Proposição 3.21, $\gamma(\beta)$ só pode ser singular no caso $\delta=1$.

Lema 3.23 A imagem $f\left(\Sigma_{\gamma(\alpha)}\right)$ é uma $\delta$-umbílica contida num hiperplano de $\mathbb{H}^{n+1}$ e os campos tangentes a $\Sigma_{\gamma(\alpha)}$ estão no núcleo do operador de Weingarten da imersão $f$.

\section{Demonstração:}

Como $\alpha \in J_{\leq 1} \backslash \stackrel{\circ}{J}_{\leq 1}$ (pela maximalidade de $I$ ), a primeira afirmação segue do Lema 3.22. Seja então, $\mathcal{H}_{\alpha}$ o hiperplano de $\mathbb{H}^{n+1}$ contendo $f\left(\Sigma_{\gamma(\alpha)}\right)$. Considere os campos (locais) unitários

$$
\left\{\begin{array}{lll}
e & \in T \Sigma_{\gamma(\alpha)}^{\perp} \cap T \mathcal{H}_{\alpha} \\
e^{\perp} & \in & T \mathcal{H}_{\alpha}^{\perp}
\end{array}\right.
$$

Temos $\xi=a e+b e^{\perp}, \operatorname{com} a^{2}+b^{2}=1$. Dado $Y \in T \Sigma_{\gamma(\alpha)}$, resulta

$$
-A_{\xi}(Y)=\widetilde{\nabla}_{Y} \xi=Y(a) e+a c Y+Y(b) e^{\perp}
$$

pois $e$ é direção umbílica e $e^{\perp}$ é paralelo.

Se $c=0$, então $A_{\xi}(Y) \in \operatorname{span}\left\{e, e^{\perp}\right\}=T \Sigma^{\perp}$, logo, sendo também tangente a $M, A_{\xi}(Y)$ é paralelo a $X$. Assim, como $r k\left(A_{\xi}\right) \leq 1, \operatorname{Im}\left(A_{\xi}\right) \subseteq \operatorname{span}\{X\}$ e $\operatorname{ker}\left(A_{\xi}\right)=\operatorname{Im}\left(A_{\xi}\right)^{\perp} \supseteq(\operatorname{span}\{X\})^{\perp}=T \Sigma_{\gamma(\alpha)}$ (com um certo abuso de linguagem).

Se $c \neq 0$, como $r k\left(A_{\xi}\right) \leq 1$, então $a=0$, pois do contrário

$$
\{Y, Z\} \text { 1.i. } \Rightarrow\{a c Y, a c Z\} \text { 1.i. } \Rightarrow \operatorname{rk}\left(A_{\xi}\right) \geq 2 \text {. }
$$

Mas $a=0 \Rightarrow b$ constante $\Rightarrow Y(a)=Y(b)=0 \quad \forall Y \in \mathfrak{X}\left(\Sigma_{\gamma(\alpha)}\right)$. Assim, também nesse caso, $T \Sigma_{\gamma(\alpha)} \subset \operatorname{ker}\left(A_{\xi}\right)$.

Observação 3.24 Se $\gamma(\beta)$ é ponto regular, então o lema acima vale também para $\Sigma_{\gamma(\beta)}$.

Lema 3.25 Se $t \in(\alpha, \beta)$, então $G(\gamma([\alpha, t]))$ é isométrico a $L_{q}^{-1}([\alpha, t])$, para um certo $q \in \mathbb{L}^{n+2}$. Se $\gamma(\beta)$ é regular, o mesmo vale para $t=\beta$. 


\section{Demonstração:}

Como $\gamma(\alpha)$ e $\gamma(t)$ são pontos regulares, $\gamma(\alpha-\epsilon, t+\epsilon)$ é uma fatia em $\gamma(\alpha)$ (cf. Proposição 1.27, levando em consideração que $(\alpha-\epsilon, t+\epsilon)$ é conexo). Pela Proposição 3.10, e pelo fato das órbitas principais serem isométricas a $\delta$-umbílicas, posto $\Gamma=G(\gamma([\alpha, t]))$, temos que $\Gamma$ é um produto torcido do tipo

$$
\Gamma=[\alpha, t] \times_{\phi} \Sigma_{\delta}
$$

onde $\Sigma_{\delta}$ é $\mathbb{S}^{n-1}(1), \mathbb{R}^{n-1}$ ou $\mathbb{H}^{n-1}(-1)$ se, respectivamente, for $\delta=1, \delta=0$ ou $\delta=-1$ (note que, nesse caso, $\delta$ representa também a curvatura seccional de $\left.\Sigma_{\delta}\right)$.

Por outro lado, indicando com $K_{s}$ a curvatura seccional da órbita de $\gamma(s)$, $\forall s \in[\alpha, t]$, tem-se, por 1.11 (pg. 11), que

$$
K_{s}=\frac{1}{\phi^{2}(s)} \delta .
$$

Agora, das propriedades do tensor de curvatura dos produtos torcidos, seguese que (cf. Observação 1.12, itens (a) e (b)):

1. $\phi^{\prime \prime}-\phi=0$

2. $K_{s}-\left(\phi^{\prime}(s) / \phi(s)\right)^{2}=-1$

Integrando a (1), tem-se que

$$
\phi(s)=A \cosh s+B \operatorname{senh} s .
$$

Já pela (2),

$$
\phi^{\prime}(s)^{2}-\phi(s)^{2}=\delta \Rightarrow B^{2}-A^{2}=\delta .
$$

Assim, tomando $c \in \mathbb{R}^{+}$tal que

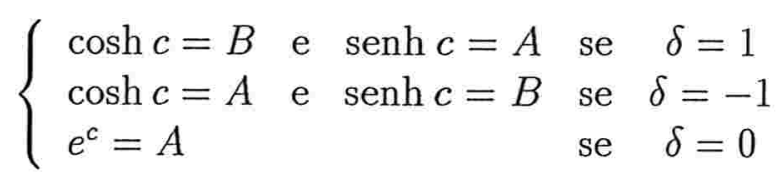

resulta

$$
\phi(s)=\left\{\begin{array}{llc}
\operatorname{senh}(c+s) & \text { se } & \delta=1 \\
\cosh (c+s) & \text { se } & \delta=-1 \\
e^{c+s} & \text { se } & \delta=0
\end{array}\right.
$$


Portanto, tendo em mente a expressão de $\mathbb{H}^{n}$ como produto torcido (cf. Seção 2.5), resulta que $\Gamma$ é isométrico a um domínio do tipo $L_{q}^{-1}([\alpha, t])$, onde $q \in \mathbb{H}^{n}, \mathbb{S}_{1}^{n}$ ou $\mathbb{V}_{+}^{n}$ se for, respectivamente $\delta=1,-1$ ou 0.

Observação 3.26 Pelo fato de $L_{q}^{-1}([a, b])$ ser um domínio anular quando $q \in \mathbb{H}^{n}$, continuaremos a chamar de domínios anulares os domínios desse tipo.

Vejamos agora um lema técnico que será usado no lema seguinte.

Lema 3.27 Se $\gamma(\beta)$ é ponto regular, seja $D^{n}=L_{q}^{-1}([\alpha, \beta])$ um domínio anular de $\mathbb{H}^{n}$ isométrico a $G(\gamma([\alpha, \beta]))$. Indiquemos com $\partial D=\partial_{\alpha} D \cup \partial_{\beta} D$ o bordo de $D$, onde $\partial_{\alpha} D$ (respectivamente $\partial_{\beta} D$ ) corresponde à órbita de $\gamma(\alpha)$ (resp. $\gamma(\beta)$ ). Dado $x \in \stackrel{D}{ }$, com $L_{q}(x) \neq 0$ se $q \in \mathbb{S}_{1}^{n}$, seja $\mathcal{H}^{n-1}$ um hiperplano de $\mathbb{H}^{n}$ passando por $x$. Então, $\mathcal{H} \cap \partial D \neq \varnothing$. Se $\gamma(\beta)$ é singular não-excepcional, tome $D^{n}=L_{q}^{-1}([\alpha, t])$, com $t \in(\alpha, \beta)$ e um hiperplano $\mathcal{H}^{n-1}$ como antes. Então, $\mathcal{H} \cap \partial_{\alpha} D \neq \varnothing$.

\section{Demonstração:}

O caso $\gamma(\beta)$ regular é bastante trivial. Basta pensar que os bordos $\partial_{\alpha} D$ e $\partial_{\beta} D$ correspondem a intersecções de $\mathbb{H}^{n}$ com subespaços afins $n$-dimensionais paralelos de $\mathbb{L}^{n+1}$, enquanto que o hiperplano $\mathcal{H}$ é determinado por um $n$ subespaço linear (portanto passando pela origem). É imediato ver que este subespaço intersecta ao menos um dos outros dois e o faz em $\mathbb{H}^{n}$. A única exceção é quando o hiperplano $\mathcal{H}$ está contido em $D$ (o que só pode acontecer se $\left.q \in \mathbb{S}_{1}^{n}\right)$, mas nesse caso teríamos $L_{q}(x)=0$.

Já o caso em que $\gamma(\beta)$ é singular não é tão trivial assim. Note que, pela Proposição 3.21 , este caso só pode ocorrer se $q \in \mathbb{H}^{n}$, isto é, as órbitas principais são esferas geodésicas. Iremos provar que

$$
\lim _{t \rightarrow \beta} \operatorname{vol}\left(\Sigma_{\gamma(t)}\right)=0 .
$$

Como conseqüencia, $\partial_{\alpha} D$ é o bordo externo de $D$ e, portanto, $\mathcal{H} \cap \partial_{\alpha} D \neq \varnothing$. 
Seja $H=G_{\gamma(\beta)}$ a isotropia em $\gamma(\beta)$, e seja $\left\{s_{i}\right\} \subset(\alpha, \beta)$ uma seqüencia convergente a $\beta$. Para cada índice $i$, defina

$$
Z_{i}=H\left(\gamma\left(s_{i}\right)\right)
$$

Note que, pelo Teorema 1.33 , as subvariedades $Z_{i}$ são difeomorfas a esferas de dimensão $k$ positiva (pois $\gamma(\beta)$ não é excepcional).

Como $G(\gamma(\alpha, \beta])$ tem curvatura constante -1 , existe uma vizinhança $U$ de $\gamma(\beta)$ (que podemos supor $H$-invariante) isométrica a um aberto de $\mathbb{H}^{n}$ (note que, sendo singular, $\gamma(\beta)$ está no interior de $G(\gamma(\alpha, \beta]))$. Seja $\psi$ essa isometria, e ponha $p=\psi(\gamma(\beta))$. Como $U$ é $H$-invariante, então, para todo $h \in H$, $\psi \circ h$ é uma isometria de $\psi(U)$. Assim, temos que $\psi\left(Z_{i}\right)$ é a esfera geodésica $S^{k}\left(p, \beta-s_{i}\right)$ de centro $p$ e raio $\left(\beta-s_{i}\right)$.

Fixados $i$ e $j$, tome a homotetia $\varphi_{i j}: \psi\left(Z_{i}\right) \rightarrow \psi\left(Z_{j}\right)$ dada por

$$
\varphi_{i j}\left(\exp _{p}\left(\beta-s_{i}\right) v\right)=\exp _{p}\left(\beta-s_{j}\right) v,
$$

onde $v \in T_{p} \mathbb{H}^{n}$ e $\|v\|=1$. Para calcular a diferencial $\mathrm{d} \varphi_{i j}$ (a fim de obter o coeficiente de homotetia), dado $y \in \psi\left(Z_{i}\right)$ e $w \in T_{y} \psi\left(Z_{i}\right)$, seja $\sigma(u)=$ $\exp _{p}\left(\beta-s_{i}\right) v(u)$ uma curva em $\psi\left(Z_{i}\right) \operatorname{com} \sigma(0)=y$ e $\dot{\sigma}(0)=w$ (isto é, $\left.(\mathrm{D} v / \mathrm{d} u)(0)=\left(1 / \operatorname{senh}\left(\beta-s_{i}\right)\right) w\right)$. Então,

$$
\begin{gathered}
\mathrm{d} \varphi_{i j}(w)=\frac{\mathrm{d}}{\mathrm{d} u}\left(\varphi_{i j} \circ \sigma\right)_{\left.\right|_{u=0}}= \\
=\frac{\mathrm{d}}{\mathrm{d} u}\left(\exp _{p}\left(\beta-s_{j}\right) v(u)\right)_{\left.\right|_{u=0}}=\operatorname{senh}\left(\beta-s_{j}\right) \frac{\mathrm{D} v}{\mathrm{~d} u}(0)=\lambda_{i j} w
\end{gathered}
$$

onde

$$
\lambda_{i j}=\frac{\operatorname{senh}\left(\beta-s_{j}\right)}{\operatorname{senh}\left(\beta-s_{i}\right)} .
$$

Assim, $\varphi_{i j}$ é uma homotetia com coeficiente $\lambda_{i j}^{2}$. Em particular, temos que

$$
\operatorname{vol}\left(\psi\left(Z_{j}\right)\right)=\lambda_{i j}^{k} \operatorname{vol}\left(\psi\left(Z_{i}\right)\right)
$$

Desta, segue-se que

$$
\lim _{s_{j} \rightarrow \beta} \operatorname{vol}\left(\psi\left(Z_{j}\right)\right)=0
$$

Resta agora provar que

$$
\lim _{s_{j} \rightarrow \beta} \operatorname{vol}\left(\psi\left(Z_{j}\right)\right)=0 \Leftrightarrow \lim _{s_{j} \rightarrow \beta} \operatorname{vol}\left(\sum_{\gamma\left(s_{j}\right)}\right)=0 .
$$


Dados, então, $i$ e $j$, seja $t \in(\alpha, \beta)$ tal que $s_{i}, s_{j} \in(\alpha, t)$. Olhando para a isometria entre $G(\gamma([\alpha, t]))$ e $[\alpha, t] \times_{\phi} \mathbb{S}^{n}(1)$, e considerando a homotetia equivariante $\theta: \Sigma_{\gamma\left(s_{i}\right)} \rightarrow \Sigma_{\gamma\left(s_{j}\right)}$ dada por $\theta\left(s_{i}, y\right)=\left(s_{j}, y\right)$, temos que o fator de homotetia é dado por

$$
\mu_{i j}=\frac{\phi^{2}\left(s_{j}\right)}{\phi^{2}\left(s_{i}\right)} .
$$

Mas, pela equivariância de $\theta$, resulta $\theta\left(Z_{i}\right)=Z_{j}$, e portanto $\mu_{i j}=\lambda_{i j}^{2}$. Assim, fixado $i$,

$$
\lim _{s_{j} \rightarrow \beta} \lambda_{i j}=0 \Leftrightarrow \lim _{s_{j} \rightarrow \beta} \mu_{i j}=0
$$

o que implica a equivalência desejada.

Lema 3.28 A imersão $f$ manda $G(\gamma([\alpha, \beta]))$ num hiperplano de $\mathbb{H}^{n+1}$.

\section{Demonstração:}

Seja $\Gamma=G(\gamma([\alpha, t]))$, com $t=\beta$ se $\gamma(\beta)$ é regular ou $t \in(\alpha, \beta)$ caso contrário. Inicialmente, suponha que $\gamma(\beta)$ é um ponto não-excepcional. Identifiquemos $\Gamma$ com o domínio anular $D=L_{q}^{-1}([\alpha, t])$ e pensemos a imersão $f_{\mid \Gamma}$ como sendo definida em $D$. Queremos provar que $f$ é totalmente geodésica, isto é, $\tau(x)=0 \quad \forall x \in D$ (no caso de $\gamma(\beta)$ ser singular, a conclusão do lema segue passando ao limite para $t \rightarrow \beta$, lembrando que o posto da segunda forma é uma função semicontínua inferiormente). Procederemos por absurdo.

Suponha que exista $x_{0} \in D^{\circ}$ tal que $\tau\left(x_{0}\right)=1$. Então existe uma vizinhança $U \subset \stackrel{D}{ }$ de $x_{0}$ onde $\tau \equiv 1$. Indicando com $A$ o operador de Weingarten de $f$, defina a distribuição

$$
\mathfrak{H}:=\{Y \in T U \mid A(Y)=0\}=\operatorname{ker}(A) .
$$

Como em cada ponto de $U$ há exatamente duas curvaturas principais distintas, a distribuição $\mathfrak{H}$ é diferenciável e involutiva em $U$ (cf. Proposição 2.3 de [Ry], pg. 372). Além do mais, $\forall Y, Z \in \mathfrak{H}$ e $V \in \mathfrak{H}^{\perp}$

$$
\begin{gathered}
\left\langle A\left(\nabla_{Y} Z\right), V\right\rangle=\left\langle\nabla_{Y} Z, A(V)\right\rangle=-\left\langle Z, \nabla_{Y} A(V)\right\rangle= \\
=-\left\langle Z,\left(\nabla_{Y} A\right) V\right\rangle-\left\langle Z, A\left(\nabla_{Y} V\right)\right\rangle=-\left\langle Z,\left(\nabla_{Y} A\right) V\right\rangle-\left\langle A(Z), \nabla_{Y} V\right\rangle=
\end{gathered}
$$




$$
\begin{gathered}
=-\left\langle Z,\left(\nabla_{Y} A\right) V\right\rangle=-\left\langle Z,\left(\nabla_{V} A\right) Y\right\rangle \quad \text { (Codazzi) } \\
=\left\langle Z, A\left(\nabla_{V} Y\right)\right\rangle=0
\end{gathered}
$$

Logo, a distribuição $\mathfrak{H}$ é também totalmente geodésica. Assim, a folha por $x_{0}$ é a interseç̧ão de $U$ com um hiperplano $\mathcal{H}^{n-1}$ de $\mathbb{H}^{n}$.

Seja agora $V$ um campo normal a $\mathcal{H}^{n-1}$ em $\mathbb{H}^{n}$ (definido globalmente em $\mathcal{H}$ ). Para cada $x \in U \cap \mathcal{H}$, resulta $A\left(V_{x}\right)=\lambda_{x} V_{x}$, com $\lambda_{x} \neq 0$.

Seja $\sigma: \mathbb{R}^{+} \rightarrow \mathcal{H}^{n-1}$ uma semi-geodésica com $\sigma(0)=x_{0}$ e tal que $\sigma\left(\mathbb{R}^{+}\right) \cap$ $\partial D \neq \varnothing$. Uma tal geodésica de fato existe por vias do Lema 3.27. Ainda, pela demonstração desse mesmo lema, fica claro que podemos supor $\sigma$ transversal a $\partial_{\alpha} D$ ou $\partial_{\beta} D$ (lembre que este último só é considerado quando $\gamma(\beta)$ é regular). Como a contradição a que chegaremos concerne o Lema 3.23, o qual vale também para $\gamma(\beta)$ regular (veja Observação 3.24), podemos supor, sem perda de generalidade, que $\sigma$ encontra $\partial_{\alpha} D$ e o faz transversalmente. Tomaremos $d>0$ tal que $\sigma(d) \in \partial_{\alpha} D$.

Defina o conjunto

$$
F=\left\{s \in[0, d] \mid A(\dot{\sigma}(u))=0 \text { e } V_{\sigma(u)} \text { é autovetor de } A \quad \forall u \in[0, s]\right\} .
$$

Note que 0 é ponto interior de $F$. Seja $\bar{s}=\sup F$ e, para $s \in[0, \bar{s}]$, seja $\rho(s)=$ $\left\langle A\left(V_{\sigma(s)}\right), V_{\sigma(s)}\right\rangle$. Então, para todo $s \in(0, \bar{s})$, resulta (tomando extensões locais de $V$ e $\dot{\sigma})$ :

$$
\begin{gathered}
\frac{\mathrm{d}}{\mathrm{d} s} \rho(s)=\left\langle\nabla_{\dot{\sigma}} A(V), V\right\rangle+\underbrace{\left\langle A(V), \nabla_{\dot{\sigma}} V\right\rangle}_{=0 \because A(V) / / V}= \\
=\left\langle\left(\nabla_{\dot{\sigma}} A\right) V, V\right\rangle-\underbrace{\left\langle A\left(\nabla_{\dot{\sigma}} V\right), V\right\rangle}_{=0}=\left\langle\left(\nabla_{V} A\right) \dot{\sigma}, V\right\rangle \quad \text { (Codazzi) } \\
=\underbrace{\left\langle\nabla_{V} A(\dot{\sigma}), V\right\rangle}_{=0 \because \dot{\sigma} \in \operatorname{ker}(A)}-\left\langle A\left(\nabla_{V} \dot{\sigma}\right), V\right\rangle=-\rho\left\langle\nabla_{V} \dot{\sigma}, V\right\rangle .
\end{gathered}
$$

Logo, posto $h=\left\langle\nabla_{V} \dot{\sigma}, V\right\rangle$,

$$
\frac{\dot{\rho}}{\rho}=-h \Rightarrow \rho=e^{-\int h}
$$

Agora, se por um lado as igualdades acima fazem sentido só para $s \in(0, \bar{s})$, por outro lado, a função $h$ está definida em toda a semi-reta real $\mathbb{R}^{+}$e, portanto, pela última igualdade, resulta

$$
\lim _{s \rightarrow \bar{s}} \rho(s) \neq 0 .
$$


Desse modo, devemos ter $\tau(\sigma(\bar{s}))=1$, o que implica, sendo $\bar{s}=\sup F$, que $\bar{s}=d$. Assim, $V_{\sigma(d)}$ é um gerador da imagem de $A$ em $T_{\sigma(d)} D$ e não é ortogonal a $\partial_{\alpha} D$ (pois $\sigma$ é transversal a esse bordo, por construção). Mas isso contradiz o Lema 3.23 .

Finalmente, suponha que $\gamma(\beta)$ seja um ponto excepcional. Lembrando da construção feita no início desta etapa, tome o recobrimento duplo $\kappa: \widetilde{\Gamma} \rightarrow \Gamma$, onde, mais uma vez, $\Gamma=G(\gamma([\alpha, \beta]))$. A ação $G \times \widetilde{\Gamma} \rightarrow \widetilde{\Gamma}$ só tem órbitas regulares e, portanto, pelo visto acima, $(f \circ \kappa)(\widetilde{\Gamma})$ está contida num hiperplano de $\mathbb{H}^{n+1}$. Logo, o mesmo vale para $f(\Gamma)=(f \circ \kappa)(\widetilde{\Gamma})$.

Teorema 3.29 Para todo $x \in \stackrel{\circ}{M}_{\leq 1}$, existe uma vizinhança $G$-invariante $U$ de $x$ tal que $f_{\left.\right|_{U}}$ é de revolução (e as órbitas são mandadas nos paralelos de $f(U))$, isto é, $\dot{M}_{\leq 1} \subset \mathcal{R}$.

\section{Demonstração:}

Aproveitando a notação usada até agora, seja $t_{0}=\left(\pi \circ \gamma_{\left.\right|_{J}}\right)^{-1}(\pi(x)) \in \stackrel{\circ}{J}_{\leq 1}$ e tome o intervalo $I=(\alpha, \beta)$ como antes. Se $\mathcal{H}^{n}$ é o hiperplano de $\mathbb{H}^{n+1}$ que contém $f(G(\gamma(I)))$ segundo o Lema 3.28 , seja $L^{n+1} \subset \mathbb{L}^{n+2}$ o subespaço linear tal que $\mathcal{H}=L \cap \mathbb{H}^{n+1}$, e seja $p \in \mathbb{S}_{1}^{n+1}$ tal que $\langle p\rangle:=\operatorname{span}\{p\}=L^{\perp}$. Evidentemente, $\mathcal{H}$ é invariante por $S O_{\langle p\rangle}^{+}$.

Por outro lado, tome $q \in L^{n+1}$ tal que $f\left(\Sigma_{\gamma(t)}\right)=L_{q}^{-1}(t) \quad \forall t \in(\alpha, \beta)$ (note que, pelo que vimos acima, $f(G(\gamma(I)))$ é um domínio anular aberto de $\mathcal{H})$. As órbitas $\Sigma_{\gamma(t)}$ são claramente invariantes por $\left(S O_{\langle p\rangle}^{+}\right)_{\langle q\rangle}$ (isto é, pelo subgrupo das isometrias de $S O_{\langle p\rangle}^{+}$que deixam fixo $\left.\langle q\rangle\right)$.

Tomando, enfim, o plano $W=\operatorname{span}\{p, q\}$, resulta que a vizinhança $U=$ $G(\gamma((\alpha, \beta)))$ de $x$ é tal que $f(U)$ é $S O_{W}^{+}$-invariante, ou seja, $f_{\left.\right|_{U}}$ é de revolução. 


\section{Conclusão (Caso $\mathcal{R} \neq \varnothing$ )}

Resumindo as duas etapas precedentes, provamos que $M_{>1} \subset \mathcal{R}$ e $\dot{M}_{\leq 1} \subset$ $\mathcal{R}$, isto é, $\mathcal{R}$ é denso em $M_{\text {reg }}$. Além do mais, provamos também que as órbitas principais imergem como umbílicas de $\mathbb{H}^{n+1}$ (cf. Lema 3.22 para órbitas que não estão em $\mathcal{R})$.

Desse modo, para cada $x \in M_{\text {reg }}$, está bem determinado o subespaço $n$-dimensional $W(x) \subset \mathbb{L}^{n+2}$ tal que

$$
f(G(x))=(f(x)+W(x)) \cap \mathbb{H}^{n+1} .
$$

Assim, podemos definir a aplicação nor : $M_{\text {reg }} \rightarrow \mathbb{G}_{2}\left(\mathbb{L}^{n+2}\right)$ de $M_{\text {reg }}$ na grassmanniana dos 2-planos de $\mathbb{L}^{n+2}$ dada por

$$
\operatorname{nor}(x)=W(x)^{\perp} .
$$

Note que, pela expressão de $W(x)$ dada pelo Teorema 2.6 , resulta que a aplicação nor é $C^{\infty}$ (no sentido que é gerada por campos locais $C^{\infty}$ ).

Lema 3.30 A aplicação nor é constante.

\section{Demonstração:}

Segue de um fato simples de topologia diferencial. Seja $X$ uma variedade conexa, $Y \subset X$ um aberto denso e $\varphi: X \rightarrow Z$ uma aplicação $C^{1}$ tal que $\varphi_{\left.\right|_{Y}}$ seja localmente constante (no nosso caso, $X=M_{\text {reg }}, Y=\mathcal{R}$ e $\varphi=$ nor). Então $\varphi$ é constante.

De fato, $\forall y \in Y, \mathrm{~d} \varphi_{y} \equiv 0$. Como $Y$ é denso em $X$, devo ter $\mathrm{d} \varphi_{x} \equiv 0$ para todo $x$ em $X$. A afirmação segue da conexidade de $X$.

Concluindo a demonstração do caso $\mathcal{R} \neq \varnothing$, temos o seguinte

Lema 3.31 Se $W$ é o "valor" constante da aplicação nor, então $f(M)$ é $S O_{W}^{+}$-invariante, isto é, $f$ é de revolução.

Demonstração:

Já sabemos que $f\left(M_{\text {reg }}\right)$ é $S O_{W}^{+}$-invariante. Seja então $p \in M \backslash M_{\text {reg }}$, e 
mostremos que $S O_{W}^{+}(f(p)) \subset f(M)$. Seja $\gamma: \mathbb{R} \rightarrow M$ uma geodésica normal com $\gamma(0)=p$. Provamos anteriormente que as $G$-órbitas principais de $M$ são mandadas, pela $f$, nas $S O_{W}^{+}$-órbitas de $f\left(\gamma(\mathbb{R}) \cap M_{r e g}\right)$. Isso significa que existe $\epsilon>0$ tal que $\gamma(0, \epsilon) \subset M_{\text {reg }}$ e tal que $f_{\left.\right|_{G(\gamma(0, \epsilon))}}$ é um mergulho. Em particular, $\overline{f(G(\gamma(0, \epsilon)))}=f(G(\gamma([0, \epsilon])))$. Então, para cada $T \in S O_{W}^{+}$

$$
\begin{aligned}
T(f(p)) & =\lim _{t \rightarrow 0} T(f(\gamma(t))) \in \overline{f(G(\gamma(0, \epsilon)))}= \\
& =f(G(\gamma([0, \epsilon]))) \subset f(M) .
\end{aligned}
$$

\subsubsection{Caso $\mathcal{R}=\varnothing$}

Passemos direto à demonstração do caso $\mathcal{R}=\varnothing$. Inicialmente, relembremos o conteúdo da Observação 3.20 (e conseqüências):

1. Nenhuma órbita principal de $M$ tem curvatura seccional constante.

2. $\tau(x) \geq 2 \quad \forall x \in M_{\text {reg }}$ e, portanto, pelos Corolários 3.15 e 3.16 temos, respectivamente:

(a) $\left(A_{\xi} X\right)(x)=\mu(x) X(x)$;

(b) $\exists \Omega^{n} \subset \mathbb{H}^{n+1}$ umbílica (que depende da órbita), com campo normal dado por $X$, tal que $f(G(x)) \subset \Omega$ com o operador de Weingarten dado por $\bar{A}_{\xi}$.

Dado então $x \in M_{\text {reg }}$, seja $\Sigma_{x}=G(x)$ e $I \times_{\phi} \Sigma_{x}$ uma vizinhança $G$ invariante de $x$. Como $X$ é autovetor de $A_{\xi}$, existem $U_{1}, \ldots, U_{n-1} \in T_{x} \Sigma_{x}$ tais que $A_{\xi}\left(U_{i}\right)=k_{i} U_{i}$. A curvatura seccional $K_{M}\left(X, U_{i}\right)$ não depende do índice $i$ (cf. Observação 1.11) e, portanto, pela equação de Gauss da imersão $M^{n} \rightarrow \mathbb{H}^{n+1}$ temos

$$
-1+\mu k_{i}=-1+\mu k_{j} \quad \forall i, j .
$$

Como $\Sigma_{x}$ não tem curvatura constante, necessariamente é $\mu=0$. Logo,

$$
\tilde{\nabla}_{X} X=\nabla_{X} X+\left\langle A_{\xi} X, X\right\rangle \xi=0
$$


ou seja, $X$ é paralelo em $\mathbb{H}^{n+1}$. Portanto, $f(I \times\{g(x)\})$ é um segmento de geodésica de $\mathbb{H}^{n+1}, \forall g \in G$.

Por outro lado, pelo item 2 (b), seja $\Omega^{n}$ uma hipersuperfície umbílica de $\mathbb{H}^{n+1}$ contendo $\Sigma_{x}$. A inclusão de $\Sigma_{x}$ em $\Omega$ é não-umbílica, pois, do contrário, $\Sigma_{x}$ seria umbílica em $\mathbb{H}^{n+1}$ e teria curvatura seccional constante. Além do mais, do fato de ser $\mu=0$, segue-se também que $r k\left(\bar{A}_{\xi}\right)=r k\left(A_{\xi}\right) \geq 2$.

Resumindo: $\Sigma_{x}$ é uma hipersuperfície homogênea e não-umbílica de $\Omega$ com número tipo maior ou igual a 2 , e $f\left(I \times_{\phi} \Sigma_{x}\right)$ é união de segmentos de geodésicas de $\mathbb{H}^{n+1}$ ortogonais a $\Omega$ em pontos de $f\left(\Sigma_{x}\right)$. Isto é, $f\left(I \times_{\phi} \Sigma_{x}\right)$ é um aberto de um oportuno exemplo NR (incluindo aí aqueles não completos, obtidos a partir de uma $n$-esfera geodésica de $\mathbb{H}^{n+1}$ - cf. Observação 3.8).

Até aqui a prova foi local. Seja agora uma geodésica normal $\gamma: \mathbb{R} \rightarrow M$, e seja $J \subset \mathbb{R}$ homeomorfo a $\pi\left(M_{\text {reg }}\right)$ (via $\pi \circ \gamma$, como já fizemos antes). Pelo que vimos na prova local, para cada $t \in J$, existe um intervalo $I(t) \subset J$ contendo $t$ tal que $f(G(\gamma(I(t))))$ é um aberto de um certo exemplo NR que indicaremos com $\Lambda_{t}$. Além disso, para cada $t \in J, f(\gamma(I(t)))$ é um segmento de geratriz de $\Lambda_{t}$.

Temos que $J=\cup\{I(t) \mid t \in J\}$, e, portanto, $f(\gamma(J))$ é união de segmentos de geodésicas de $\mathbb{H}^{n+1}$. Mas, sendo $f$ de classe $C^{\infty}$ e $J$ conexo, $f(\gamma(J))$ deve ser um único segmento $\left(C^{\infty}\right)$ de geodésica, o mesmo valendo para $f(g(\gamma(J))) \quad \forall g \in G$. Isto é, $f(g(\gamma(J)))$ (ao variar de $g$ ) são segmentos de geratrizes de um mesmo exemplo NR $\Lambda$. Assim, $I(t)=I(s)=J$ e $\Lambda_{t}=\Lambda_{s}=\Lambda \quad \forall t, s \in J$, e $f\left(M_{r e g}\right) \subset \Lambda$.

Por fim, note que $\gamma^{-1}\left(M_{\text {reg }}\right)$ é união disjunta de intervalos de $\mathbb{R}$ e que $\mathbb{R} \backslash \gamma^{-1}\left(M_{\text {reg }}\right)$, se não for vazio, é constituído de pontos isolados. Logo, pelo mesmo argumento de diferenciabilidade de $f$, para cada $g \in G, f(g(\gamma(\mathbb{R})))$ é uma geodésica de $\mathbb{H}^{n+1}$, ou mais precisamente, uma geratriz de $\Lambda$ (que, por conter então geratrizes definidas em toda a reta, não pode ser do tipo "gerado" por esferas geodésicas). Portanto, $f(M)=\Lambda$, concluindo a prova deste caso e, principalmente, a do Teorema 3.9. 


\subsection{Discussão Comparativa}

Parece-nos interessante fazer uma comparação entre os casos euclidiano e hiperbólico, já que o trabalho nasceu da idéia de estender a esse último resultados obtidos para o primeiro.

A primeira pergunta natural é: e se $G$ for compacto? Nesse caso as órbitas são compactas, o que só acontece, pelo Teorema que provamos, no caso das hipersuperfícies de revolução do tipo esférico. Lembre que, no caso das rotacionais esféricas, há de fato um eixo de revolução determinado pela intersecção do plano fixo com $\mathbb{H}^{n+1}$. Isto significa que o grupo que age sobre a hipersuperfície é, na verdade, o subgrupo das isometrias de $\mathbb{H}^{n+1}$ que deixa fixo esse eixo. De tudo isso, segue-se que o Teorema 3.1 (Seixas) vale, com as mesmas hipóteses, também no caso de hipersuperfícies do espaço hiperbólico.

A segunda questão que se coloca é, num certo sentido, o outro lado da questão anterior: o que acontece no caso euclidiano se $G$ não é compacto? Será que o Teorema 3.9, com definições e hipóteses análogas (principalmente a de que o grupo não necessariamente é compacto), vale também no caso de hipersuperfícies do espaço euclidiano? A resposta, nesse caso é, de certo modo, sim e não. Vejamos a questão mais de perto.

Antes de mais nada, torna-se necessária uma extensão do conceito de hipersuperfície de revolução. Em [dCDa], onde nos baseamos para a definição de rotacional do espaço hiperbólico, os autores definem as hipersuperfícies de revolução de formas espaciais reais, incluindo, portanto, as rotacionais do $\mathbb{R}^{n+1}$. Mas estas coincidem (e por que haveria de ser diferente?) com a definição usual. No nosso caso, como estamos "pensando ao contrário" (isto é, estendendo conceitos do espaço hiperbólico para o espaço euclidiano), seria razoável considerar como hipersuperfícies de revolução também os cilindros sobre curvas planas (que, mesmo euclidianamente, podem ser vistos como rotacionais com eixo no infinito).

Por outro lado, porém, os cilindros sobre curvas planas são hipersuperfícies altamente deformáveis, sobre as quais não há como esperar nenhum tipo de teorema de rigidez. De fato, voltando a pensar na validade do Teorema 3.9 no caso euclidiano, qualquer hipótese de controle sobre a planaridade exclui imediatamente esse tipo de hipersuperfície (mal foram criadas, já são 
sumariamente eliminadas). Em outras palavras, no caso da hipersuperfície considerada resultar ser de revolução, o será no sentido usual.

Resta analisar a existência de exemplos $N R$ em $\mathbb{R}^{n+1}$. De forma análoga, pode-se construí-los partindo de esferas ou de hiperplanos. No primeiro caso obtêm-se cones sobre subvariedades (homogêneas, não-umbílicas de posto maior ou igual a 2) da esfera que, assim como os cones do caso hiperbólico, só interessam localmente.

Já no segundo caso, obtemos de fato exemplos NR completos. Assim, numa possível extensão do Teorema, a hipersuperfície considerada poderia resultar ser um exemplo NR desse tipo. Cabe, porém, uma observação de caráter qualitativo. Esse exemplo NR pode, sim, ser visto como uma hipersuperfície de co-homogeneidade 1, mas é mais do que isso: trata-se, na verdade, de uma hipersupefície homogênea "disfarçada" de co-homogeneidade 1. Os exemplos NR de $\mathbb{H}^{n+1}$ são, por outro lado, "puramente" de co-homogeneidade 1.

De certo modo, portanto, parece que uma extensão do Teorema 3.9 a hipersuperfícies de $\mathbb{R}^{n+1}$ de fato existe: seria o Teorema 3.1 de Seixas com, a mais, o fato de que a não compacidade do grupo resultaria no exemplo NR acima (o uso do verbo "parecer" aqui não é mero exercício de modéstia, e sim uma explicitação do fato de que essas linhas são uma "conversa sobre o tema", e não uma demonstração). Esse é o lado "sim" da resposta. O lado "não" da resposta é uma pura especulação qualitativa. Concerne o fato de que uma tal extensão não melhora, na nossa opinião, o nosso conhecimento sobre as hipersupefícies de co-homogeneidade 1 do espaço euclidiano.

Mas, enfim, qual a diferença entre os dois casos? No início, acreditávamos ser o fato de trabalhar com grupos não compactos. Num certo sentido, é isso mesmo. Mas essa diferença só é significativa no espaço hiperbólico, principalmente por ele ter três tipos essenciais de hipersuperfícies umbílicas, enquanto que o espaço euclidiano só tem uma (não totalmente geodésica). 


\section{Referências Bibliográficas}

[AlAl] A. V. Alekseevsky e D. V. Alekseevsky. Riemannian $g$-manifold with one-dimensional orbit space. Ann. Global Anal. Geom., 11:197-211, 1993.

[Al] C. B. Allendoerfer. Rigidity for spaces of class greater than one. Amer. J. of Math., 61:633-644, 1939.

[ACN] A. C. Asperti, H. P. do Castro, e M. H. Noronha. Compact homogeneous Einstein manifolds in codimension two. Note di Matematica, 16(1):9-19, 1996.

[AMN] A. C. Asperti, F. Mercuri, e M. H. Noronha. Cohomogeneity one manifolds and hypersurfaces of revolution. Boll. Un. Mat. Ital. B (7), 11(2, suppl.):199-215, 1997.

[Be] R. Beez. Zur theorie des krümmungsmasses von mannigfaltigkeiten höhere ordnung. Zeit. für Math. und Physik, 21:373-401, 1876.

[Ber] J. Berndt. Homogeneous hypersurfaces in hyperbolic spaces. Math. Z., 229:589-600, 1998.

[Br] G. E. Bredon. Introduction to Compact Transformation Groups. Academic Press, New York, 1972. Pure and Applied Mathematics, Vol. 46.

[dC] M. P. do Carmo. Geometria riemanniana, volume 10 do Projeto Euclides. Instituto de Matemática Pura e Aplicada, Rio de Janeiro, 1979.

[dCDa] M. P. do Carmo e M. Dajczer. Rotation hypersurfaces in spaces of constant curvature. Trans. Amer. Math. Soc., 277(2):685-709, 1983. 
[CaNo] H. P. do Castro e M. H. Noronha. Homogeneous submanifolds of codimension two. Geom. Dedicata, 78(1):89-110, 1999.

[Ce] T. E. Cecil. A characterization of metric spheres in hyperbolic space by Morse theory. Tohoku Math. J., II. Ser., 26:341-351, 1974.

[CeRy] T. E. Cecil e P. J. Ryan. Distance functions and umbilic submanifolds of hyperbolic space. Nagoya Math. J., 74:67-75, 1979.

[CKKT] Y.-W. Choe, H. S. Kim, I.-B. Kim, e R. Takagi. Rigidity theorems for real hypersurfaces in a complex projective space. Hokkaido Math. J., 25(3):433-451, 1996.

[Da] M. Dajczer. Submanifolds and isometric immersions, volume 13 de Mathematics Lecture Series. Publish or Perish Inc., Houston, TX, 1990. Baseado em notas preparadas por Mauricio Antonucci, Gilvan Oliveira, Paulo Lima-Filho e Rui Tojeiro.

[Fu] R. Fukuoka. Hipersuperfícies no espaço euclidiano com condições sobre a geometria intrínseca. Tese de Doutorado, IMECCUNICAMP, 1999.

[Ha] C. E. Harle. Rigidity of hypersurfaces of constant scalar curvature. J. Differential Geom., 5:85-111, 1971.

[HsLa] W.-Y. Hsiang e H.B. Lawson Jr. Minimal submanifolds of low cohomogeneity. J. Differ. Geom., 5:1-38, 1971.

[Ki] W. Killing. Die nicht-Euklidischen Raumformen in Analytische Behandlung. Teubner, Leipzig, 1885.

[KKT] I.-B. Kim, B. H. Kim, e R. Takagi. The rigidity for real hypersurfaces in a complex projective space. Tohoku Math. J. (2), 50(4):531536, 1998.

[Ko1] S. Kobayashi. Compact homogeneous hypersurfaces. Trans. Amer. Math. Soc., 88:137-143, 1958.

[Ko2] S. Kobayashi. Transformation Groups in Differential Geometry, volume 70 de Ergebnise der Mathematik und ihrer Grenzgebiete. Springer-Verlag, Berlin, Heidelberg, New York, 1972. 
[KoNo] S. Kobayashi e K. Nomizu. Foundations of Differential Geometry II, volume 15-II de Interscience Tracts in Pure and Applied Mathematics. Interscience Publishers, New York, Chichester, Brisbane, Toronto, 1969.

[MPS] F. Mercuri, F. Podestà, e J. A. P. Seixas. Topology of cohomogeneity one hypersurfaces of euclidean spaces. Preprint.

[Mo] P. S. Mostert. On a compact Lie group acting on a manifold. Ann. of Math., II. Ser., 65:447-455, 1957.

[NaTa] T. Nagano e T. Takahashi. Homogeneous hypersurfaces in euclidean spaces. J. Math. Soc. Japan, 12:1-7, 1960.

[Nö] S. Nölker. Isometric immersions of warped products. Differ. Geom. Appl., 6(1):1-30, 1996.

[O'N] B. O'Neill. Semi-Riemannian geometry with applications to relativity, volume 103 de Pure and Applied Mathematics. Academic Press, NewYork - London, 1983.

[PaTe] R. S. Palais e C.-L. Terng. Critical point theory and submanifold geometry. Springer-Verlag, Berlin, 1988.

[PoSp] F. Podestà e A. Spiro. Cohomogeneity one manifolds and hypersurfaces of the Euclidean space. Ann. Global Anal. Geom., 13(2):169184, 1995.

[PoTh] F. Podestà e G. Thorbergsson. Polar actions on rank-one symmetric spaces. J. Differential Geom., 53(1):131-175, 1999.

[Ra] J. G. Ratcliffe. Foundations of hyperbolic manifolds., volume 149 of Graduate Texts in Mathematics. Springer-Verlag, Berlin, 1994.

[Ry] P. J. Ryan. Homogeneity and some curvature conditions for hypersurfaces. Tôhoku Math. J. (2), 21:363-388, 1969.

[Se] J. A. P. Seixas. Hipersuperfícies de cohomogeneidade um do espaço euclidiano. Tese de Doutorado, IMECC-UNICAMP, 1996.

[St] N. Steenrod. Topology of Fibre Bundles. Princeton Univ. Press, Princeton, 1951. 
[SuTk] Y. J. Suh e R. Takagi. A rigidity for real hypersurfaces in a complex projective space. Tôhoku Math. J. (2), 43(4):501-507, 1991.

[Suz] C. A. Suzuki. Hipersuperfícies de rotação em espaços de curvatura constante. Dissertação de Mestrado, Instituto de Matemática e Estatística, USP, 1989.

[Tk] R. Takagi. On homogeneous real hypersurfaces in a complex projective space. Osaka J. Math., 10:495-506, 1973.

[TaTk] R. Takagi e T. Takahashi. On the principal curvatures of homogeneous hypersurfaces in a sphere. Differential Geometry, in honor of K. Yano, Kinokuniya, Tokyo, pages 469-481, 1972.

[Ta1] T. Takahashi. Homogeneous hypersurfaces in spaces of constant curvature. J. Math. Soc. Japan, 22:395-410, 1970.

[Ta2] T. Takahashi. An isometric immersion of a homogeneous Riemannian manifold of dimension 3 in the hyperbolic space. J. Math. Soc. Japan, 23:649-661, 1971. 